\title{
Twist decomposition of nonlocal light-cone operators II: General tensors of 2nd rank
}

\author{
Bodo Geyer* and Markus Lazar ${ }^{\dagger}$ \\ Center for Theoretical Studies and Institute of Theoretical Physics, \\ Leipzig University, Augustusplatz 10, D-04109 Leipzig, Germany
}

November 3, 2018

\begin{abstract}
A group theoretical procedure, introduced earlier in [6], to decompose bilocal light-ray operators into (harmonic) operators of definite twist is applied to the case of arbitrary $2 \mathrm{nd}$ rank tensors. As a generic example the bilocal gluon operator is considered which gets contributions of twist-2 up to twist-6 from four different symmetry classes characterized by corresponding Young tableaux; also the twist decomposition of the related vector and scalar operators is considered. In addition, we extend these results to various trilocal light-ray operators, like the Shuryak-Vainshtein, the threegluon and the four-quark operators, which are required for the consideration of higher-twist distribution amplitudes. The present results rely on the knowledge of harmonic tensor polynomials of any order $n$ which have been determined up to the case of 2 nd rank symmetric tensor for arbitrary space-time dimension.
\end{abstract}

*e-mail: geyer@itp.uni-leipzig.de

†e-mail: lazar@itp.uni-leipzig.de 


\section{Introduction}

Any careful quantum field theoretical analysis of the experimental data for the different light-cone dominated QCD-processes (for recent experiments and analyses, see [1, 2]) necessarily requires a rigorous twist decomposition of the various nonlocal light-ray operators appearing in the theoretical set up of these processes. The matrix elements of these special operators are related to the distribution amplitudes (or the hadronic wave functions) being necessary for the phenomenological description of the above mentioned processes. Moreover, their anomalous dimensions determine the $Q^{2}$-evolution of the distribution amplitudes.

The notion of twist has been originally introduced for local operators by Gross and Treiman [3] as a geometric quantity, twist $\tau=$ (canonical) dimension $d$-spin $j$, which is directly related to the irreducible representations of the Lorentz group.? The twist decomposition of the local operators of some well-defined tensor structure corresponds directly to their decomposition into irreducible tensor representations of the Lorentz group. These representations are uniquely (up to equivalence) determined by their symmetry classes, i.e., Young frames, and by the (anti)symmetrization defined by corresponding Young operators, i.e., specific Young tableaux.

In the case of nonlocal tensor operators, being given as (infinite) towers of local tensor operators of growing rank, the definition of (geometric) twist is more subtle. For that reason another notion of (dynamical) twist, being related to the LC-quantization in the infinite momentum frame [4], has been introduced [5] by counting powers of $1 / Q$ which, from a phenomenological point of view, is quite advantageous. However, this different notion of twist has serious theoretical disadvantages: It is not Lorentz invariant and, therefore, not immediately related to geometric twist; furthermore, it is process-dependent because it is only applicable to matrix elements of the corresponding $\mathrm{LC}$-operators and not to the operators itself.

In order to be able to disentangle the twist content of nonlocal LC-operators which are relevant for various hard QCD processes a systematic group theoretical study has been started in an earlier paper [6], thereafter cited as I. There, bilocal quark operators up to (antisymmetric) second rank have been considered. Here, we continue this study for general tensor operators of 2 nd rank. We carry out the complete twist decomposition of all relevant LC-operators, e.g., bilocal gluon operators, trilocal quark-gluon correlation operators (related to so-called ShuryakVainshtein operators) and four-fermion operator, as well as multilocal quark and gluon operators. Thereby, generalized harmonic polynomials being tensors up to rank 2 are introduced and the interior derivative on the light-cone is used.

The paper is organized as follows. In Chapt. 2 we shortly review the general

${ }^{1}$ Obviously, if $d$ is taken as scale dimension of the operators it is not related to the generators of the Lorentz group but to those of the subgroup $S O(2 h) \otimes \mathbb{R}_{+}$of the conformal group in $2 h$-dimensions. Thus a local operator with definite twist is a irreducible finite dimensional representation of the group $S O(2 h) \otimes \mathbb{R}_{+}$. 
method introduced in Part I. In Chapt. 3 we give the complete twist decomposition of the various gluonic bilocal light-ray tensor operators of second rank being specified by different symmetry classes; their twist content ranges from 2 up to 6 . In Chapt. 4 we extend these results with minor modifications to the trilocal lightray operators mentioned above. Any of these nonlocal operators consists of an infinite tower of local operators which are characterized by equal twist. Appendix A contains some material about the tensorial harmonic functions.

\section{General procedure}

Let us now shortly review the general procedure, introduced in Part I, of how to decompose arbitrary bilocal LC-operators into harmonic operators of definite twist. Those nonlocal operators which are relevant for the virtual Compton scattering [7] are obtained by the nonlocal LC expansion [8, 9] of the (renormalized) time-ordered product of two electromagnetic hadronic currents:

$$
\mathcal{R} T\left(J^{\mu}\left(y+\frac{x}{2}\right) J^{\nu}\left(y-\frac{x}{2}\right) S\right) \underset{x^{2} \rightarrow 0}{\approx} \int \mathrm{d}^{2} \underline{\kappa} C_{\Gamma}^{\mu \nu}\left(x^{2}, \underline{\kappa}\right) \mathcal{R} T\left(\mathcal{O}^{\Gamma}\left(y+\kappa_{1} \tilde{x}, y+\kappa_{2} \tilde{x}\right) S\right)+\cdots
$$

where $\Gamma=\left\{1, \gamma_{\mu}, \sigma_{\mu \nu} ; \gamma_{5}, \gamma_{\mu} \gamma_{5}, \sigma_{\mu \nu} \gamma_{5}\right\}$ indicates the tensor structure of the nonlocal quark operators and $\tilde{x}$ is a light-like vector related to $x$, cf. [8, 10, 6]. The unrenormalized nonlocal (flavour singlet) operators at $y=0$ are given by

$$
\mathcal{O}^{\Gamma}\left(\kappa_{1} \tilde{x}, \kappa_{2} \tilde{x}\right)=\bar{\psi}\left(\kappa_{1} \tilde{x}\right) \Gamma U\left(\kappa_{1} \tilde{x}, \kappa_{2} \tilde{x}\right) \psi\left(\kappa_{2} \tilde{x}\right)
$$

with the path ordered phase factor

$$
U\left(\kappa_{1} \tilde{x}, \kappa_{2} \tilde{x}\right)=\mathcal{P} \exp \left\{\mathrm{i} g \int_{\kappa_{1}}^{\kappa_{2}} \mathrm{~d} \tau \tilde{x}^{\mu} A_{\mu}(\tau \tilde{x})\right\},
$$

ensuring gauge invariance. As is well-known, under renormalization these operators mix with appropriate chiral-even (or chiral-odd) bilocal gluonic tensor operators, eventually multiplied by appropriate tensors built up from $\tilde{x}$ :

$$
\begin{aligned}
& G_{\alpha \beta}\left(\kappa_{1} \tilde{x}, \kappa_{2} \tilde{x}\right)=F_{\alpha}{ }^{\rho}\left(\kappa_{1} \tilde{x}\right) U\left(\kappa_{1} \tilde{x}, \kappa_{2} \tilde{x}\right) F_{\beta \rho}\left(\kappa_{2} \tilde{x}\right), \\
& \widetilde{G}_{\alpha \beta}\left(\kappa_{1} \tilde{x}, \kappa_{2} \tilde{x}\right)=F_{\alpha}{ }^{\rho}\left(\kappa_{1} \tilde{x}\right) U\left(\kappa_{1} \tilde{x}, \kappa_{2} \tilde{x}\right) \widetilde{F}_{\beta \rho}\left(\kappa_{2} \tilde{x}\right),
\end{aligned}
$$

where $F_{\alpha \beta}$ and $\widetilde{F}_{\alpha \beta}=\frac{1}{2} \epsilon_{\alpha \beta \mu \nu} F^{\mu \nu}$ are the gluon field strength and its dual, respectively. Here, the phase factors are to be taken in the adjoint representation.

In general, the twist decomposition of an arbitrary bilocal light-ray operator may be formulated for any space-time dimension $D=2 h$. Let us denote such operators for arbitrary values of $x$ as follows:

$$
\mathcal{O}^{\Gamma}\left(\kappa_{1} x, \kappa_{2} x\right)=\Phi^{\prime}\left(\kappa_{1} x\right) \Gamma U\left(\kappa_{1} x, \kappa_{2} x\right) \Phi\left(\kappa_{2} x\right)
$$

where, suppressing any indices indicating the group representations, $\Phi$ generically denotes the various local fields, e.g., scalars $(d=h-1)$, Dirac spinors $\left(d=h-\frac{1}{2}\right)$ 
as well as gauge field strength $(d=h) . A_{\mu}$ is the gauge potential having dimension $d=h-1$. Furthermore, $\Gamma$ labels the tensor structure as well as additional quantum numbers, if necessary.

Now, the twist decomposition of operators (2.5) consists of the following steps:

(1) Taylor expansion of the nonlocal operators for arbitrary values of $x$ at the point $y=0$ into an infinite series of local tensor operators having definite rank $n$ and canonical dimension $d$ :

$$
\mathcal{O}^{\Gamma}\left(\kappa_{1} x, \kappa_{2} x\right)=\sum_{n=0}^{\infty} \frac{1}{n !} x^{\mu_{1}} \ldots x^{\mu_{n}}\left[\Phi^{\prime}(y) \Gamma \stackrel{\leftrightarrow}{D}_{\mu_{1}}\left(\kappa_{1}, \kappa_{2}\right) \ldots \stackrel{\leftrightarrow}{D}_{\mu_{n}}\left(\kappa_{1}, \kappa_{2}\right) \Phi(y)\right]_{y=0}
$$

with the generalized covariant derivatives

$$
\begin{aligned}
\stackrel{\leftrightarrow}{D}_{\mu}\left(\kappa_{1}, \kappa_{2}\right) & \equiv \kappa_{1} \overleftarrow{D}_{\mu}+\kappa_{2} \vec{D}_{\mu} \\
\vec{D}_{\mu}=\vec{\partial}_{\mu}^{y}+\mathrm{i} g A_{\mu}(y), & \overleftarrow{D}_{\mu}=\overleftarrow{\partial}_{\mu}^{y}-\mathrm{i} g A_{\mu}(y)
\end{aligned}
$$

(2) Decomposition of local operators with respect to irreducible tensor representations of the Lorentz group $S O(2 h-1,1)$ or, equivalently, the orthogonal group $S O(2 h)$. 7 These representations are built up by traceless tensors of rank $m$ whose symmetry class is determined by some (normalized) Young operators $\mathcal{Y}_{[m]}=\left(f_{[m]} / m !\right) \mathcal{Q P}$, where $[m]=\left(m_{1}, m_{2}, \ldots m_{r}\right)$ with $m_{1} \geq m_{2} \geq \ldots \geq m_{r}$ and $\sum_{i=1}^{r} m_{i}=m$ denotes the corresponding Young pattern. $\mathcal{P}$ and $\mathcal{Q}$, as usual, denote symmetrization and antisymmetrization with respect to that pattern. The allowed Young patterns for $S O(2 h)$, which because of the tracelessness are restricted by $\ell_{1}+\ell_{2} \leq 2 h$ ( $\ell$ : length of columns of $[m]$ ), are for a fixed value of $m$ :

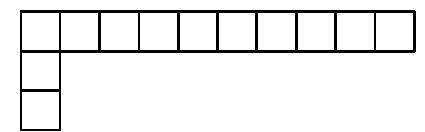

$$
j=m-2, m-3, m-4, \ldots
$$



(Here, we depicted only those patterns which appear for $h=2$ where $\ell_{1}+\ell_{2} \leq 4$.) Loosely speaking, the lower spins in the above series correspond to the various trace terms which define, in general, reducible higher twist operators. These

\footnotetext{
${ }^{2}$ The corresponding representations are related through analytic continuation, cf. [11, 12, 6]
} 
higher twist operators again have to be decomposed according to the considered symmetry classes.

Now, two remarks are in order. At first, this decomposition of the local operators appearing in (2.6) into irreducible components is independent of the special values of $\kappa_{1}, \kappa_{2}$. Therefore, we may choose $\kappa_{1}=0, \kappa_{2}=\kappa$ and factor out $\kappa^{n}$ for every term in eq. (2.6), thus simplifying the explicit computations below. At the end of the computations the general values of $\kappa_{i}$ may be re-installed. Furthermore, if these irreducible local tensor operators are multiplied by $x^{\mu_{1}} \ldots x^{\mu_{n}}$, as it is necessary according to eq. (2.6), then harmonic (tensorial) polynomials of order $n$ appear whose remaining tensor structure results from $\Gamma$. For a detailed exposition of these objects, see Appendix A.

(3) Resummation of the local operators $\mathcal{O}_{\Gamma n}^{\tau}(x)$ belonging to the same symmetry class (for any $n$ ) and having equal twist $\tau$. That infinite tower of local operators creates a nonlocal operator of definite twist,

$$
\mathcal{O}_{\Gamma}^{\tau}(0, \kappa x)=\bigoplus_{n=0}^{\infty} \frac{\kappa^{n}}{n !} \mathcal{O}_{\Gamma n}^{\tau}(x) .
$$

Obviously, from a group theoretical point of view, this nonlocal operator is built up as direct sum of irreducible local tensor operators. The nonlocal operators $\mathcal{O}_{\Gamma}^{\tau}(0, \kappa x)$ are harmonic tensor functions. Now, putting together all contributions, i.e., including the higher twist contributions resulting from the trace terms, we get the (infinite) twist decomposition of the nonlocal operator we started with:

$$
\mathcal{O}_{\Gamma}(0, x)=\bigoplus_{\tau=\tau_{\min }}^{\infty} \mathcal{O}_{\Gamma}^{\tau}(0, x) .
$$

(4) Finally, the projection onto the light-cone, $x \rightarrow \tilde{x}$, leads to the required lightcone operator with well defined geometric twist. However, since the harmonic tensor polynomials essentially depend on (infinite) sums of powers of $x^{2}$ and $\square$ as well as some specific differential operators in front of it, in that limit only a finite number of terms survive:

$$
\mathcal{O}_{\Gamma}\left(\kappa_{1} \tilde{x}, \kappa_{2} \tilde{x}\right)=\bigoplus_{\tau=\tau_{\min }}^{\tau_{\max }} \mathcal{O}_{\Gamma}^{\tau}\left(\kappa_{1} \tilde{x}, \kappa_{2} \tilde{x}\right) .
$$

The resulting light-ray operators of definite twist, which have been rewritten for general arguments, are tensor functions on the light-cone which fulfil another kind of tracelessness conditions to be formulated with the help of the interior (on the cone) derivative. - Let us remark that step (3) and (4) can be interchanged without changing the result.

The advantages of our method are: First, these nonlocal operators of definite twist are Lorentz covariant tensors (contrary to the phenomenological concept of twist). Second, this twist decomposition is unique, process- and modelindependent. Furthermore, the twist decomposition is independent from the dimension $2 h$ of space-time. 


\section{Twist decomposition of a general $2 \mathrm{nd}$ rank tensor operator}

This Chapter is devoted to the twist decomposition of a general 2nd rank tensor operator. For simplicity, we restrict ourselves to four dimensional space-time $(h=$ 2 ). Furthermore, since the twist decomposition is independent from the chirality we demonstrate it only for the chiral-even gluon operator (2.3). In addition, we make the special choice $\kappa_{1}=0, \kappa_{2}=\kappa$, thereby also simplifying the generalized covariant derivatives, eq. (2.7), to the usual ones:

$$
\begin{gathered}
G_{\alpha \beta}(0, \kappa x)=F_{\alpha}{ }^{\rho}(0) U(0, \kappa x) F_{\beta \rho}(\kappa x)=\sum_{n=0}^{\infty} \frac{\kappa^{n}}{n !} x^{\mu_{1}} \ldots x^{\mu_{n}} G_{\alpha \beta\left(\mu_{1} \ldots \mu_{n}\right)} \\
\text { with } \quad G_{\alpha \beta\left(\mu_{1} \ldots \mu_{n}\right)}=\left.F_{\alpha}{ }^{\rho}(y) D_{\left(\mu_{1}\right.}^{y} \ldots D_{\left.\mu_{n}\right)}^{y} F_{\beta \rho}(y)\right|_{y=0}
\end{gathered}
$$

here the symbol $(\ldots)$ denotes symmetrization of the enclosed indices.

The local tensor operators $G_{\alpha \beta\left(\mu_{1} \ldots \mu_{n}\right)}$ decompose according to the Young patterns (i) to (iv) and possible higher ones (i.e., for $m_{1}+m_{2}=n+2, m_{2} \geq 3$ ):

$$
\begin{aligned}
G_{\alpha \beta\left(\mu_{1} \ldots \mu_{n}\right)}= & G_{\alpha \beta\left(\mu_{1} \ldots \mu_{n}\right)}^{(\mathrm{i})}+\alpha_{n+1} G_{\alpha \beta\left(\mu_{1} \ldots \mu_{n}\right)}^{(\mathrm{ii})} \\
& +\beta_{n} G_{\alpha \beta\left(\mu_{1} \ldots \mu_{n}\right)}^{(\mathrm{iii})}+\gamma_{n} G_{\alpha \beta\left(\mu_{1} \ldots \mu_{n}\right)}^{(\mathrm{iv})}+\ldots,
\end{aligned}
$$

with the (nontrivial) normalization coefficients $f_{[m]} / m$ ! of the Young operators given by $\alpha_{n+1}=2(n+1) /(n+2)$ for $[m]=(n+1,1), \beta_{n}=3 n /(n+2)$ for $[m]=(n, 1,1)$ and $\gamma_{n}=4(n-1) /(n+1)$ for $[m]=(n, 2)$. The corresponding Clebsch-Gordan series in terms of representations $\left(j_{1}, j_{2}\right)$ of the Lorentz group is

$$
\begin{aligned}
\left(\frac{1}{2}, \frac{1}{2}\right) \otimes\left(\frac{1}{2}, \frac{1}{2}\right) & \otimes\left(\left(\frac{n}{2}, \frac{n}{2}\right) \oplus\left(\frac{n-2}{2}, \frac{n-2}{2}\right) \oplus \ldots\right) \\
= & \left(\frac{n+2}{2}, \frac{n+2}{2}\right) \oplus\left(\left(\frac{n+2}{2}, \frac{n}{2}\right) \oplus\left(\frac{n}{2}, \frac{n+2}{2}\right)\right) \oplus\left(\frac{n}{2}, \frac{n}{2}\right) \\
& \oplus\left(\left(\frac{n+2}{2}, \frac{n-2}{2}\right) \oplus\left(\frac{n-2}{2}, \frac{n+2}{2}\right)\right) \\
& \oplus\left(\left(\frac{n}{2}, \frac{n-2}{2}\right) \oplus\left(\frac{n-2}{2}, \frac{n}{2}\right)\right) \oplus\left(\frac{n-2}{2}, \frac{n-2}{2}\right) \oplus \ldots ;
\end{aligned}
$$

the corresponding tensor spaces will be denoted by $\mathbf{T}\left(j_{1}, j_{2}\right)$. In the last line of eq. (3.3) such representations are listed down which correspond to higher twist contributions contained in the trace terms of symmetry classes (i) - (iv). The canonical (or scale) dimension $d$ of the local operator (3.2) is $n+4$, and the spin of the various contributions in (3.3) ranges from $n+2$ up to 1 or 0 if $n$ is even or odd, respectively; therefore, the local operators with well-defined twist are irreducible tensors of the Lorentz group or, equivalently, of the group $S L(2, \mathbb{C}) \otimes \mathbb{R}_{+}$, having scale dimension $d$.

In general, according to the spin content and the rank of the corresponding local tensor operators, the nonlocal operator (3.1) for arbitrary $x$ contains contributions of twist ranging from $\tau=2$ until $\tau=\infty$. However, after projection onto 
the light-cone, $x \rightarrow \tilde{x}$, this infinite series terminates at least at $\tau=6$ since higher order terms are proportional to $x^{2}$.

In order to be more explicit, as well as for later use, we introduce the (anti)symmetrization with respect to $\alpha$ and $\beta$ and we define the following nonlocal operators:

$$
\begin{aligned}
G_{\alpha \beta}^{ \pm}(0, \kappa x) & :=\frac{1}{2}\left(G_{\alpha \beta}(0, \kappa x) \pm G_{\beta \alpha}(0, \kappa x)\right) \\
G_{\alpha}^{ \pm}(0, \kappa x) & :=x^{\beta} G_{\alpha \beta}^{ \pm}(0, \kappa x) \equiv G_{\alpha \bullet}^{ \pm}(0, \kappa x) \\
G(0, \kappa x) & :=x^{\alpha} x^{\beta} G_{\alpha \beta}^{+}(0, \kappa x) .
\end{aligned}
$$

In fact, the twist decomposition of these bilocal light-ray operators reads:[?

$$
\begin{aligned}
G_{\alpha \beta}^{+}(0, \kappa \tilde{x})= & G_{(\alpha \beta)}^{\mathrm{tw} 2}(0, \kappa \tilde{x})+G_{(\alpha \beta)}^{\mathrm{tw} 3}(0, \kappa \tilde{x})+G_{(\alpha \beta)}^{\mathrm{tw} 4}(0, \kappa \tilde{x}) \\
& +G_{(\alpha \beta)}^{\mathrm{tw} 5}(0, \kappa \tilde{x})+G_{(\alpha \beta)}^{\mathrm{tw} 6}(0, \kappa \tilde{x}), \\
G_{\alpha \beta}^{-}(0, \kappa \tilde{x})= & G_{[\alpha \beta]}^{\mathrm{tw} 3}(0, \kappa \tilde{x})+G_{[\alpha \beta]}^{\mathrm{tw} 4}(0, \kappa \tilde{x})+G_{[\alpha \beta]}^{\mathrm{tw} 5}(0, \kappa \tilde{x}), \\
G_{\alpha}^{+}(0, \kappa \tilde{x})= & G_{(\alpha \bullet)}^{\mathrm{tw} 2}(0, \kappa \tilde{x})+G_{(\alpha \bullet)}^{\mathrm{tw} 3}(0, \kappa \tilde{x})+G_{(\alpha \bullet)}^{\mathrm{tw} 4}(0, \kappa \tilde{x}), \\
G_{\alpha}^{-}(0, \kappa \tilde{x})= & G_{[\alpha \bullet]}^{\mathrm{tw} 3}(0, \kappa \tilde{x})+G_{[\alpha \bullet]}^{\mathrm{tw} 4}(0, \kappa \tilde{x}), \\
G(0, \kappa \tilde{x})= & G^{\mathrm{tw} 2}(0, \kappa \tilde{x}) .
\end{aligned}
$$

The various twist contributions individually decompose further according to the symmetry classes which may contribute. The explicit expressions for generic tensor operators are given in the following subsections, where it will be shown that Young patterns (i) and (ii) as well as (iv) contribute to the symmetric tensor operators and related vector and scalar operators, and Young patterns (ii) and (iii) contribute to the antisymmetric tensor operators and related vector operators. Special tensor operators are considered in Chapt. 4.

\subsection{Tensor operators of symmetry class (i)}

\subsubsection{Construction of nonlocal symmetric class-(i) operators of defi- nite twist}

Let us start with the simplest case of the totally symmetric traceless tensors, and their contractions with $x$, which have twist $\tau=2$ and are contained in the tensor space $\mathbf{T}\left(\frac{n+2}{2}, \frac{n+2}{2}\right)$. They have symmetry class (i) and are uniquely characterized by the following standard tableau (with normalizing factor 1 ):

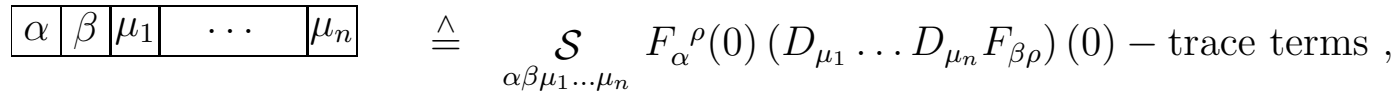

The irreducible local twist-2 operator reads

$$
G_{\left(\alpha \beta \mu_{1} \ldots \mu_{n}\right)}^{\mathrm{tw} 2(\mathrm{i})}=F_{(\alpha}^{\rho}(0) D_{\mu_{1}} \ldots D_{\mu_{n}} F_{\beta) \rho}(0)-\text { trace terms . }
$$

\footnotetext{
${ }^{3}$ We use the notation $A_{(\alpha \beta)} \equiv \frac{1}{2}\left(A_{\alpha \beta}+A_{\beta \alpha}\right)$ and $A_{[\alpha \beta]} \equiv \frac{1}{2}\left(A_{\alpha \beta}-A_{\beta \alpha}\right)$.
} 
Let us postpone the determination of the trace terms and make the resummation to the corresponding nonlocal operator in advance. This is obtained, at first, by contracting with $x^{\mu_{1}} \ldots x^{\mu_{n}}$ and rewriting in the following form:

$$
G_{(\alpha \beta) n}^{\mathrm{tw} 2(\mathrm{i})}(x)=x^{\mu_{1}} \ldots x^{\mu_{n}} G_{\left(\alpha \beta \mu_{1} \ldots \mu_{n}\right)}^{\mathrm{tw} 2 \mathrm{i})}=\frac{1}{(n+2)(n+1)} \partial_{\alpha} \partial_{\beta} \stackrel{\circ}{G}_{n+2}(x),
$$

with the denotation

$$
\begin{aligned}
& \stackrel{\circ}{G}_{n+2}(x)=G_{n+2}(x)-\text { trace terms } \\
& G_{n+2}(x) \equiv x^{\mu} x^{\nu} F_{\mu}{ }^{\rho}(0)(x D)^{n} F_{\nu \rho}(0) .
\end{aligned}
$$

Here, $\stackrel{\circ}{G}_{n+2}(x)$ is a harmonic polynomial of order $n+2$, cf. eq. (A.5), Appendix $\mathrm{A}$ :

$$
\stackrel{\circ}{G}_{n+2}(x)=H_{n+2}^{(4)}\left(x^{2} \mid \square\right) G_{n+2}(x) \equiv \sum_{k=0}^{\left[\frac{n+2}{2}\right]} \frac{(n+2-k) !}{(n+2) ! k !}\left(\frac{-x^{2}}{4}\right)^{k} \square^{k} G_{n+2}(x) .
$$

Then, using $((n+2)(n+1))^{-1}=\int_{0}^{1} \mathrm{~d} \lambda(1-\lambda) \lambda^{n}$ and the integral representation of Euler's beta function we obtain the nonlocal twist-2 tensor operator: 1

$$
G_{(\alpha \beta)}^{\mathrm{tw} 2}(0, \kappa x)=\sum_{n=0}^{\infty} \frac{\kappa^{n}}{n !} G_{(\alpha \beta) n}^{\mathrm{tw} 2(\mathrm{i})}(x)=\partial_{\alpha} \partial_{\beta} \int_{0}^{1} \mathrm{~d} \lambda(1-\lambda) \stackrel{\circ}{G}(0, \kappa \lambda x),
$$

with

$$
\stackrel{\circ}{G}(0, \kappa x)=G(0, \kappa x)+\sum_{k=1}^{\infty} \int_{0}^{1} \mathrm{~d} t t\left(\frac{-x^{2}}{4}\right)^{k} \frac{\square^{k}}{k !(k-1) !}\left(\frac{1-t}{t}\right)^{k-1} G(0, \kappa t x) .
$$

The operator $G_{(\alpha \beta)}^{\mathrm{tw} 2}(0, \kappa x)$ satisfies the conditions of a harmonic tensor function:

$$
g^{\alpha \beta} G_{(\alpha \beta)}^{\mathrm{tw} 2}(0, \kappa x)=0, \quad \square G_{(\alpha \beta)}^{\mathrm{tw} 2}(0, \kappa x)=0, \quad \partial^{\alpha} G_{(\alpha \beta)}^{\mathrm{tw} 2}(0, \kappa x)=0 .
$$

\subsubsection{Reduction to vector and scalar operators}

Now, we specify to the nonlocal vector and scalar operators. Multiplying eq. (3.12) by $x^{\beta}$ and observing the equality $\left(x \partial_{x}\right) \stackrel{\circ}{G}(0, \kappa \lambda x)=\left(\lambda \partial_{\lambda}+2\right) \stackrel{\circ}{G}(0, \kappa \lambda x)$ gives the twist-2 vector operator,

$$
G_{(\alpha \bullet)}^{\mathrm{tw} 2}(0, \kappa x)=\partial_{\alpha} \int_{0}^{1} \mathrm{~d} \lambda \lambda \stackrel{\circ}{G}(0, \kappa \lambda x),
$$

which satisfies the conditions

$$
G_{(\alpha \bullet)}^{\mathrm{tw} 2}(0, \kappa x)=0, \quad \partial^{\alpha} G_{(\alpha \bullet)}^{\mathrm{tw} 2}(0, \kappa x)=0
$$

\footnotetext{
${ }^{4}$ Here, and in the following, we omit the indication of the symmetry class of the nonlocal twist-2 operators because only totally symmetric tensors contribute. However, the trace terms being of higher twist must be classified according to their symmetry type.
} 
Multiplying with $x^{\alpha} x^{\beta}$ gives the twist-2 scalar operator,

$$
G^{\mathrm{tw} 2}(0, \kappa x)=\stackrel{\circ}{G}(0, \kappa x)
$$

which, by definition, satisfies the condition

$$
\square G^{\mathrm{tw} 2}(0, \kappa x)=0 .
$$

The expression (3.13) for the scalar operator already has been given by Balitsky and Braun 13 .

Comparing eqs. (3.12), (3.15) and (3.17) we may recognize that in the case of symmetry class (i) the tensor and vector operators are obtained from the scalar operator by very simple operations. Furthermore, we observe how in the case of the scalar operator the trace terms - being proportional to $x^{2}$ - are to be subtracted from $G(0, \kappa x)$ in order to make that operator traceless. In the case of vector and tensor operators such subtraction, because of the appearance of the derivatives, is more complicated.

\subsubsection{Projection onto the light-cone}

Let us now project onto the light-cone and, at the same time, also extend to the case of general values $\left(\kappa_{1}, \kappa_{2}\right)$. Because of the derivatives $\partial_{\alpha}$ and $\partial_{\beta}$, appearing in eq. (3.12), only the terms with $k=1,2$ in eq. (3.13) contribute. The final expression for the symmetric twist-2 light-cone tensor operator is given by

$$
\begin{aligned}
G_{(\alpha \beta)}^{\mathrm{tw} 2}\left(\kappa_{1} \tilde{x}, \kappa_{2} \tilde{x}\right)= & \left.\partial_{\alpha} \partial_{\beta} \int_{0}^{1} \mathrm{~d} \lambda(1-\lambda) G\left(\kappa_{1} \lambda x, \kappa_{2} \lambda x\right)\right|_{x=\tilde{x}}-G_{(\alpha \beta)}^{>(\mathrm{i})}\left(\kappa_{1} \tilde{x}, \kappa_{2} \tilde{x}\right), \\
G_{(\alpha \beta)}^{>(\mathrm{i})}\left(\kappa_{1} \tilde{x}, \kappa_{2} \tilde{x}\right)= & \int_{0}^{1} \mathrm{~d} \lambda\left\{(1-\lambda+\lambda \ln \lambda)\left(\frac{1}{2} g_{\alpha \beta}+x_{(\alpha} \partial_{\beta)}\right) \square\right. \\
& \left.+\frac{1}{4}(2(1-\lambda)+(1+\lambda) \ln \lambda) x_{\alpha} x_{\beta} \square^{2}\right\}\left.G\left(\kappa_{1} \lambda x, \kappa_{2} \lambda x\right)\right|_{x=\tilde{x}}
\end{aligned}
$$

The operator $G_{(\alpha \beta)}^{>(\mathrm{i})}\left(\kappa_{1} \tilde{x}, \kappa_{2} \tilde{x}\right)$ contains the higher twist contributions which have to be subtracted from the first term, eq. (3.19), in order to make the whole expression traceless. In order to disentangle the different terms of well-defined twist we observe that the tensor operator (3.20) consists of scalar and vector parts. Here, an operator being contained in the trace terms is called a scalar and vector part, respectively, if the expression multiplied by $g_{\alpha \beta}, x_{\alpha}$ or $x_{\beta}$ is a scalar, like $\square G\left(\kappa_{1} x, \kappa_{2} x\right)$ or $\partial^{\mu} G_{\mu}\left(\kappa_{1} x, \kappa_{2} x\right)$, and a vector, like $\partial_{\beta} G\left(\kappa_{1} x, \kappa_{2} x\right)$ or $\partial_{\beta} \partial^{\mu} G_{\mu}\left(\kappa_{1} x, \kappa_{2} x\right)$, respectively. The scalar operators, $\left.\square G\left(\kappa_{1} x, \kappa_{2} x\right)\right|_{x=\tilde{x}}$ and $\left.\square^{2} G\left(\kappa_{1} x, \kappa_{2} x\right)\right|_{x=\tilde{x}}$, occurring in eq. (3.20) already have well-defined twist $\tau=4$ and $\tau=6$, respectively. To ensure that the vector operator $\left.\partial_{\beta} \square G\left(\kappa_{1} x, \kappa_{2} x\right)\right|_{x=\tilde{x}}$ also obtains well-defined twist, it is necessary to subtract its own trace terms which are of twist $\tau=6$.

5 Strictly speaking, $x_{\alpha}$ and $x_{\beta}$ as well as $g_{\alpha \beta}$ which are necessary for the dimension and tensor structure of the whole expression do not belong to the higher twist operator itself. 
The higher twist operators contained in the trace terms of the twist-2 tensor operator are the following:

$$
\begin{aligned}
G_{(\alpha \beta)}^{>(\mathrm{i})}\left(\kappa_{1} \tilde{x}, \kappa_{2} \tilde{x}\right)= & G_{(\alpha \beta)}^{\mathrm{tw} 4(\mathrm{i}) \mathrm{a}}\left(\kappa_{1} \tilde{x}, \kappa_{2} \tilde{x}\right)+G_{(\alpha \beta)}^{\mathrm{tw} 4(\mathrm{i}) \mathrm{b}}\left(\kappa_{1} \tilde{x}, \kappa_{2} \tilde{x}\right) \\
& +G_{(\alpha \beta)}^{\mathrm{tw} 6(\mathrm{i}) \mathrm{a}}\left(\kappa_{1} \tilde{x}, \kappa_{2} \tilde{x}\right)+G_{(\alpha \beta)}^{\mathrm{tw} 6(\mathrm{i}) \mathrm{b}}\left(\kappa_{1} \tilde{x}, \kappa_{2} \tilde{x}\right),
\end{aligned}
$$

with

$$
\begin{aligned}
G_{(\alpha \beta)}^{\mathrm{tw} 4(\mathrm{i}) \mathrm{a}}\left(\kappa_{1} \tilde{x}, \kappa_{2} \tilde{x}\right)= & \left.\frac{1}{2} g_{\alpha \beta} \square \int_{0}^{1} \mathrm{~d} \lambda(1-\lambda+\lambda \ln \lambda) G\left(\kappa_{1} \lambda x, \kappa_{2} \lambda x\right)\right|_{x=\tilde{x}}, \\
G_{(\alpha \beta)}^{\mathrm{tw} 4(\mathrm{i}) \mathrm{b}}\left(\kappa_{1} \tilde{x}, \kappa_{2} \tilde{x}\right)= & \left.x_{(\alpha} \partial_{\beta)} \square \int_{0}^{1} \mathrm{~d} \lambda(1-\lambda+\lambda \ln \lambda) G\left(\kappa_{1} \lambda x, \kappa_{2} \lambda x\right)\right|_{x=\tilde{x}} \\
& -G_{(\alpha \beta)}^{\mathrm{tw} 6(\mathrm{i}) \mathrm{b}}\left(\kappa_{1} \tilde{x}, \kappa_{2} \tilde{x}\right),
\end{aligned}
$$

and

$$
\begin{aligned}
& G_{(\alpha \beta)}^{\mathrm{tw} 6(\mathrm{i}) \mathrm{a}}\left(\kappa_{1} \tilde{x}, \kappa_{2} \tilde{x}\right)=\left.\frac{1}{4} x_{\alpha} x_{\beta} \square^{2} \int_{0}^{1} \mathrm{~d} \lambda(2(1-\lambda)+(1+\lambda) \ln \lambda) G\left(\kappa_{1} \lambda x, \kappa_{2} \lambda x\right)\right|_{x=\tilde{x}}, \\
& G_{(\alpha \beta)}^{\mathrm{tw} 6(\mathrm{i}) \mathrm{b}}\left(\kappa_{1} \tilde{x}, \kappa_{2} \tilde{x}\right)=\left.\frac{1}{4} x_{\alpha} x_{\beta} \square^{2} \int_{0}^{1} \mathrm{~d} \lambda\left(\frac{(1-\lambda)^{2}}{\lambda}-\frac{1-\lambda^{2}}{2 \lambda}-\lambda \ln \lambda\right) G\left(\kappa_{1} \lambda x, \kappa_{2} \lambda x\right)\right|_{x=\tilde{x}} .
\end{aligned}
$$

Obviously, by inspection of the spin content of the symmetry type (i), the local twist -4 and twist -6 operators carry the representation $\mathbf{T}\left(\frac{n}{2}, \frac{n}{2}\right)$ and $\mathbf{T}\left(\frac{n-2}{2}, \frac{n-2}{2}\right)$, respectively.

Finally, completing the twist decomposition of the symmetric nonlocal tensor operator, we write down the symmetric twist-4 light-cone tensor operator contained in the expression $(3.20)$

$$
\begin{aligned}
G_{(\alpha \beta)}^{\mathrm{tw} 4(\mathrm{i})}\left(\kappa_{1} \tilde{x}, \kappa_{2} \tilde{x}\right) & =G_{(\alpha \beta)}^{\mathrm{tw} 4(\mathrm{i}) \mathrm{a}}\left(\kappa_{1} \tilde{x}, \kappa_{2} \tilde{x}\right)+G_{(\alpha \beta)}^{\mathrm{tw} 4(\mathrm{i}) \mathrm{b}}\left(\kappa_{1} \tilde{x}, \kappa_{2} \tilde{x}\right) \\
& =\int_{0}^{1} \mathrm{~d} \lambda\left\{(1-\lambda+\lambda \ln \lambda)\left(\frac{1}{2} g_{\alpha \beta}+x_{(\alpha} \partial_{\beta)}\right)\right. \\
& \left.-\frac{1}{4}\left(\frac{(1-\lambda)^{2}}{\lambda}-\frac{1-\lambda^{2}}{2 \lambda}-\lambda \ln \lambda\right) x_{\alpha} x_{\beta} \square^{2}\right\}\left.G\left(\kappa_{1} \lambda x, \kappa_{2} \lambda x\right)\right|_{x=\tilde{x}},
\end{aligned}
$$

whereas the symmetric twist-6 light-cone operator contained in (3.20) reads

$$
\begin{aligned}
G_{(\alpha \beta)}^{\mathrm{tw} 6(\mathrm{i})}\left(\kappa_{1} \tilde{x}, \kappa_{2} \tilde{x}\right) & =G_{(\alpha \beta)}^{\mathrm{tw6}(\mathrm{i}) \mathrm{a}}\left(\kappa_{1} \tilde{x}, \kappa_{2} \tilde{x}\right)+G_{(\alpha \beta)}^{\mathrm{tw6}(\mathrm{i}) \mathrm{b}}\left(\kappa_{1} \tilde{x}, \kappa_{2} \tilde{x}\right) \\
& =\left.\frac{1}{4} x_{\alpha} x_{\beta} \square^{2} \int_{0}^{1} \mathrm{~d} \lambda\left(\frac{1-\lambda^{2}}{2 \lambda}+\ln \lambda\right) G\left(\kappa_{1} \lambda x, \kappa_{2} \lambda x\right)\right|_{x=\tilde{x}} .
\end{aligned}
$$

\subsubsection{Vector and scalar light-ray operators}

Contracting eq. (3.19) with $\tilde{x}^{\beta}$, making use of formula $\left(x \partial_{x}\right) f(\lambda x)=\lambda \partial_{\lambda} f(\lambda x)$ and performing the partial integrations we obtain the final version of the twist-2 light-cone vector operator,

$$
G_{(\alpha \bullet)}^{\mathrm{tw} 2}\left(\kappa_{1} \tilde{x}, \kappa_{2} \tilde{x}\right)=\left.\int_{0}^{1} \mathrm{~d} \lambda \lambda\left[\partial_{\alpha}+\frac{1}{2}(\ln \lambda) x_{\alpha} \square\right] G\left(\kappa_{1} \lambda x, \kappa_{2} \lambda x\right)\right|_{x=\tilde{x}},
$$


which already has been used in [0], and the twist-4 light-cone vector operator,

$$
G_{(\alpha \bullet)}^{\mathrm{tw} 4(\mathrm{i})}\left(\kappa_{1} \tilde{x}, \kappa_{2} \tilde{x}\right)=-\left.\frac{1}{2} x_{\alpha} \square \int_{0}^{1} \mathrm{~d} \lambda \lambda(\ln \lambda) G\left(\kappa_{1} \lambda x, \kappa_{2} \lambda x\right)\right|_{x=\tilde{x}} .
$$

In order to obtain the scalar twist-2 light-ray operator we multiply (3.24) by $\tilde{x}^{\alpha}$. Then, the twist-4 part vanishes and the remaining twist-2 operator restores the scalar operator (compare eq. (3.11)),

$$
G^{\mathrm{tw} 2}\left(\kappa_{1} \tilde{x}, \kappa_{2} \tilde{x}\right)=G\left(\kappa_{1} \tilde{x}, \kappa_{2} \tilde{x}\right) .
$$

Let us point to the fact that the trace of the original gluon tensor, $g^{\alpha \beta} G_{\alpha \beta}\left(\kappa_{1} \tilde{x}, \kappa_{2} \tilde{x}\right)$, is a twist-4 scalar operator. It is contained in $G_{(\alpha \beta)}^{\mathrm{tw} 4(\mathrm{i}) \mathrm{a}}\left(\kappa_{1} \tilde{x}, \kappa_{2} \tilde{x}\right)$ as well as similar expressions occurring below, cf. also eq. (3.83).

\subsubsection{Condition of tracelessness on the cone}

Finally, it should be remarked, that the conditions (3.14), (3.16) and (3.18), if translated into the corresponding ones containing derivatives with respect to $\tilde{x}$, no longer hold for the light-cone operators. This is clear because, by projecting onto the light-cone, part of the original structure of the operators has been lost. Nevertheless, the conditions of tracelessness of the light-cone operators may be formulated by using the interior derivative on the light-cone [14, 15] which has been used extensively for the construction of local conformal operators [16, 17]. In four dimensions it is given by

$$
\mathrm{d}_{\alpha} \equiv(1+\tilde{x} \tilde{\partial}) \tilde{\partial}_{\alpha}-\frac{1}{2} \tilde{x}_{\alpha} \tilde{\partial}^{2} \quad \text { with } \quad \tilde{\partial}_{\alpha} \equiv \frac{\partial}{\partial \tilde{x}^{\alpha}},
$$

and has the following properties

$$
\mathrm{d}^{2}=0, \quad\left[\mathrm{~d}_{\alpha}, \mathrm{d}_{\beta}\right]=0 \quad \text { and } \quad \mathrm{d}_{\alpha} \tilde{x}^{2}=\tilde{x}^{2}\left(\mathrm{~d}_{\alpha}+2 \tilde{\partial}_{\alpha}\right) .
$$

Then, the conditions of tracelessness simplify, namely, they read:

$$
g^{\alpha \beta} G_{(\alpha \beta)}^{\mathrm{tw} 2}\left(\kappa_{1} \tilde{x}, \kappa_{2} \tilde{x}\right)=0, \quad \mathrm{~d}^{\alpha} G_{(\alpha \beta)}^{\mathrm{tw} 2}\left(\kappa_{1} \tilde{x}, \kappa_{2} \tilde{x}\right)=0,
$$

as well as

$$
\mathrm{d}^{\alpha} G_{(\alpha \bullet)}^{\mathrm{tw} 2}\left(\kappa_{1} \tilde{x}, \kappa_{2} \tilde{x}\right)=0 .
$$

Analogous conditions hold for the light-cone operators of definite twist in the case of symmetry classes (ii) - (iv) obtained below.

In passing we remark that the interior derivative may be used in defining more directly totally symmetric local light-cone operators [18]. For example it holds

$$
\begin{aligned}
G_{(\alpha \beta) n}^{\mathrm{tw} 2(\mathrm{i})}(\tilde{x}) & =\frac{1}{(n+2)^{2}(n+1)^{2}} \mathrm{~d}_{\alpha} \mathrm{d}_{\beta} G_{n+2}(\tilde{x}), \\
G_{(\alpha \bullet) n}^{\mathrm{tw} 2(\mathrm{i})}(\tilde{x}) & =\frac{1}{(n+2)^{2}} \mathrm{~d}_{\alpha} G_{n+2}(\tilde{x}) .
\end{aligned}
$$

However, in case of symmetry types (ii) - (iv) the corresponding expressions should be more involved. 


\subsection{Tensor operators of symmetry class (ii)}

\subsubsection{Construction of nonlocal (anti)symmetric class-(ii) operators of definite twist: Young tableau A}

Now we consider tensor operators, and their contractions with $x$, having symmetry class (ii) and whose local twist- 3 parts are contained in $\mathbf{T}\left(\frac{n+2}{2}, \frac{n}{2}\right) \oplus \mathbf{T}\left(\frac{n}{2}, \frac{n+2}{2}\right)$. Contrary to the totally symmetric case we have different possibilities to put the tensor indices into the corresponding Young pattern. Without presupposing any symmetry of indices $\alpha$ and $\beta$ we should start with the following Young tableau:

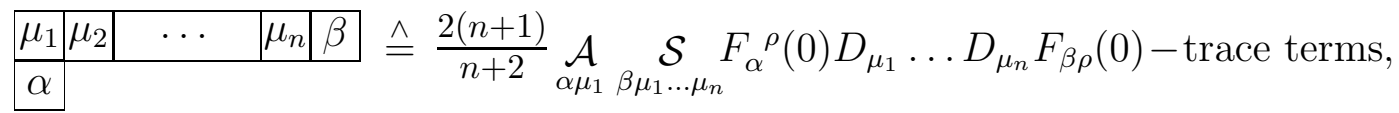

with normalizing factor $\alpha_{n+1}$. (The tableau with $\alpha \leftrightarrow \beta$ will be considered thereafter.) Denoting this symmetry behaviour by (iiA) we may write the local twist-3 tensor operator as follows:

$$
\begin{aligned}
G_{\alpha \beta \mu_{1} \ldots \mu_{n}}^{\mathrm{tw} 3(\mathrm{iiA})}=\frac{1}{n+2}\{ & F_{\alpha}{ }^{\rho}(0) D_{\left(\mu_{1}\right.} \ldots D_{\left.\mu_{n}\right)} F_{\beta \rho}(0)+F_{\alpha}{ }^{\rho}(0) D_{\beta} D_{\left(\mu_{2}\right.} \ldots D_{\mu_{n}} F_{\left.\mu_{1}\right) \rho}(0) \\
& +\sum_{l=2}^{n} F_{\alpha}{ }^{\rho}(0) D_{\left(\mu_{1}\right.} \ldots D_{\mu_{l-1}} D_{|\beta|} D_{\mu_{l+1}} \ldots D_{\mu_{n}} F_{\left.\mu_{l}\right) \rho}(0) \\
& \left.-\left(\alpha \leftrightarrow \mu_{1}\right)\right\}- \text { trace terms } .
\end{aligned}
$$

Proceeding in the same manner as in the last Subsection we multiply by $x^{\mu_{1}} \ldots x^{\mu_{n}}$ and obtain:

$$
G_{\alpha \beta n}^{\mathrm{tw} 3(\mathrm{iiA})}(x)=\frac{1}{n(n+2)}\left(\delta_{\alpha}^{\mu}(x \partial)-x^{\mu} \partial_{\alpha}\right){\stackrel{\stackrel{\circ}{G}}{G_{\mu \bullet \mid n+1}}}(x)
$$

with

$$
\begin{aligned}
\stackrel{\circ}{G}_{\mu \bullet \mid n+1}(x) & \equiv x^{\nu} \stackrel{\circ}{G}_{\mu \nu \mid n}(x) \\
& =\left\{\delta_{\mu}^{\alpha}-\frac{1}{(n+2)^{2}}\left[x_{\mu} \partial^{\alpha}(x \partial)-\frac{1}{2} x^{2} \partial_{\mu} \partial^{\alpha}\right]\right\} H_{n+1}^{(4)}\left(x^{2} \mid \square\right) G_{0 \bullet \mid n+1}(x) .
\end{aligned}
$$

Here, $\stackrel{\circ}{G}_{\mu \bullet \mid n+1}(x)$ is the harmonic vector polynomial of order $n+1$ (see, Eq. (A.7), Appendix A) and the symmetry behaviour of class (ii) is obtained through the differential operator in front of it. Now, using $((n+2) n)^{-1}=\int_{0}^{1} \mathrm{~d} \lambda\left(1-\lambda^{2}\right) \lambda^{n} /(2 \lambda)$ and Euler's beta function we sum up to obtain the following nonlocal twist-3 tensor operator:

$$
G_{\alpha \beta}^{\mathrm{tw} 3(\mathrm{iiA})}(0, \kappa x)=\int_{0}^{1} \mathrm{~d} \lambda \frac{1-\lambda^{2}}{2 \lambda}\left(\delta_{\alpha}^{\mu}(x \partial)-x^{\mu} \partial_{\alpha}\right) \partial_{\beta} \stackrel{\circ}{G}_{\mu \bullet}(0, \kappa \lambda x),
$$


with the nonlocal traceless (vector) operator

$$
\begin{aligned}
\stackrel{\circ}{G}_{\alpha \bullet}(0, \kappa x)= & G_{\alpha \bullet}(0, \kappa x)+\sum_{k=1}^{\infty} \int_{0}^{1} \mathrm{~d} t\left(\frac{-x^{2}}{4}\right)^{k} \frac{\square^{k}}{k !(k-1) !}\left(\frac{1-t}{t}\right)^{k-1} G_{\alpha \bullet}(0, \kappa t x) \\
& -\left[x_{\alpha} \partial^{\mu}(x \partial)-\frac{1}{2} x^{2} \partial_{\alpha} \partial^{\mu}\right] \\
& \times \sum_{k=0}^{\infty} \int_{0}^{1} \mathrm{~d} \tau \tau \int_{0}^{1} \mathrm{~d} t t\left(\frac{-x^{2}}{4}\right)^{k} \frac{\square^{k}}{k ! k !}\left(\frac{1-t}{t}\right)^{k} G_{\mu \bullet}(0, \kappa \tau t x) .
\end{aligned}
$$

The following decomposition into symmetric and antisymmetric part is useful for the further calculations

$$
G_{\alpha \beta}^{\mathrm{tw} 3(\mathrm{iiA})}(0, \kappa x)=G_{[\alpha \beta]}^{\mathrm{tw} 3(\mathrm{iiA})}(0, \kappa x)+G_{(\alpha \beta)}^{\mathrm{tw} 3(\mathrm{iiA})}(0, \kappa x),
$$

with

$$
\begin{aligned}
G_{[\alpha \beta]}^{\mathrm{tw} 3(\mathrm{iiA})}(0, \kappa x) & =\int_{0}^{1} \mathrm{~d} \lambda \lambda \delta_{[\alpha}^{\mu} \partial_{\beta]} \stackrel{\circ}{G}_{\mu \bullet}(0, \kappa \lambda x) \\
G_{(\alpha \beta)}^{\mathrm{tw} 3(\mathrm{iiA})}(0, \kappa x) & =\int_{0}^{1} \mathrm{~d} \lambda \frac{1}{\lambda}\left(\delta_{(\alpha}^{\mu} \partial_{\beta)}-\frac{1-\lambda^{2}}{2} \partial_{\alpha} \partial_{\beta} x^{\mu}\right) \stackrel{\circ}{G}_{\mu \bullet}(0, \kappa \lambda x) \\
& =\int_{0}^{1} \mathrm{~d} \lambda \frac{1-\lambda^{2}}{2 \lambda}\left(\delta_{(\alpha}^{\mu}(x \partial)-x^{\mu} \partial_{(\alpha}\right) \partial_{\beta} \stackrel{\circ}{G}_{\mu \bullet}(0, \kappa \lambda x) .
\end{aligned}
$$

Again, these operators are harmonic tensor functions. The conditions of tracelessness for the tensor operator are

$$
\begin{array}{rlrl}
g^{\alpha \beta} G_{\alpha \beta}^{\mathrm{tw} 3(\mathrm{iiA})}(0, \kappa x) & =0, & & \square G_{\alpha \beta}^{\mathrm{tw} 3(\mathrm{iiA})}(0, \kappa x)=0, \\
\partial^{\alpha} G_{\alpha \beta}^{\mathrm{tw} 3(\mathrm{iiA})}(0, \kappa x)=0, & & \partial^{\beta} G_{\alpha \beta}^{\mathrm{tw} 3(\mathrm{iiA})}(0, \kappa x)=0 .
\end{array}
$$

\subsubsection{Reduction to vector operators}

The corresponding twist- 3 vector operator is obtained from eq. (3.27) by multiplication with $x^{\beta}$ :

$$
G_{\alpha \bullet}^{\mathrm{tw} 3(\mathrm{iiA})}(0, \kappa x)=\int_{0}^{1} \mathrm{~d} \lambda \lambda\left(\delta_{\alpha}^{\mu}(x \partial)-x^{\mu} \partial_{\alpha}\right) \stackrel{\circ}{G_{\mu \bullet}}(0, \kappa \lambda x) .
$$

Obviously, a corresponding scalar operator does not exist.

This vector operator fulfils the following conditions of tracelessness

$$
\square G_{\alpha \bullet}^{\mathrm{tw} 3(\mathrm{iiA})}(0, \kappa x)=0, \quad \partial^{\alpha} G_{\alpha \bullet}^{\mathrm{tw} 3(\mathrm{iiA})}(0, \kappa x)=0 .
$$




\subsubsection{Projection onto the light-cone}

(a) The calculation of the antisymmetric tensor operator $G_{[\alpha \beta]}^{\mathrm{tw} 3(\mathrm{iiA})}\left(\kappa_{1} \tilde{x}, \kappa_{2} \tilde{x}\right)$ on the light-cone is similar to that of $M_{[\alpha \beta]}^{\mathrm{tw} 2}\left(\kappa_{1} \tilde{x}, \kappa_{2} \tilde{x}\right)$ in Part I; for the details we refer to it. The resulting expression is:

$$
\begin{aligned}
G_{[\alpha \beta]}^{\mathrm{tw} 3(\mathrm{iiA})}\left(\kappa_{1} \tilde{x}, \kappa_{2} \tilde{x}\right)= & \left.\int_{0}^{1} \mathrm{~d} \lambda \lambda \delta_{[\alpha}^{\mu} \partial_{\beta]} G_{\mu \bullet}\left(\kappa_{1} \lambda \tilde{x}, \kappa_{2} \lambda \tilde{x}\right)\right|_{x=\tilde{x}}-G_{[\alpha \beta]}^{>(\mathrm{iiA})}\left(\kappa_{1} \tilde{x}, \kappa_{2} \tilde{x}\right), \\
G_{[\alpha \beta]}^{>(\mathrm{iiA})}\left(\kappa_{1} \tilde{x}, \kappa_{2} \tilde{x}\right)= & \frac{1}{2} \int_{0}^{1} \mathrm{~d} \lambda(1-\lambda)\left\{\left.\left(2 x_{[\alpha} \partial_{\beta]} \partial^{\mu}-x_{[\alpha} \delta_{\beta]}^{\mu} \square\right) G_{\mu \bullet}\left(\kappa_{1} \lambda x, \kappa_{2} \lambda x\right)\right|_{x=\tilde{x}}\right. \\
& \left.-\left.(1-\lambda+\lambda \ln \lambda) x_{[\alpha} \partial_{\beta]} \square G\left(\kappa_{1} \lambda x, \kappa_{2} \lambda x\right)\right|_{x=\tilde{x}}\right\}
\end{aligned}
$$

$G_{[\alpha \beta]}^{>(\mathrm{iiA})}\left(\kappa_{1} \tilde{x}, \kappa_{2} \tilde{x}\right)$ contains twist-4 and twist- 5 contributions, but twist -6 contributions do not appear due to $x_{[\alpha} x_{\beta]}=0$. The higher twist operator (3.37) contains two vector operators multiplied by $x_{\alpha}$ and $x_{\beta}$, respectively. For their twist decomposition one has to take into account Young pattern (i) as well as (ii). The procedure is analogous to the decomposition of the vector operator $O_{\alpha}\left(\kappa_{1} \tilde{x}, \kappa_{2} \tilde{x}\right)$ made in Part I. After a straightforward calculation we obtain

$$
\begin{aligned}
G_{[\alpha \beta]}^{>(\mathrm{iiA})}\left(\kappa_{1} \tilde{x}, \kappa_{2} \tilde{x}\right)= & G_{[\alpha \beta]}^{\mathrm{tw} 4(\mathrm{iiA}) \mathrm{a}}\left(\kappa_{1} \tilde{x}, \kappa_{2} \tilde{x}\right)+G_{[\alpha \beta]}^{\mathrm{tw} 4(\mathrm{iiA}) \mathrm{b}}\left(\kappa_{1} \tilde{x}, \kappa_{2} \tilde{x}\right) \\
& +G_{[\alpha \beta]}^{\mathrm{tw} 5(\mathrm{iiA}) \mathrm{a}}\left(\kappa_{1} \tilde{x}, \kappa_{2} \tilde{x}\right)
\end{aligned}
$$

with

$$
\begin{aligned}
& G_{[\alpha \beta]}^{\mathrm{tw} 4(\mathrm{iiA}) \mathrm{a}}\left(\kappa_{1} \tilde{x}, \kappa_{2} \tilde{x}\right)=\left.\frac{1}{2} x_{[\alpha} \partial_{\beta]} \partial^{\mu} \int_{0}^{1} \mathrm{~d} \lambda \frac{1-\lambda^{2}}{\lambda} G_{\mu \bullet}\left(\kappa_{1} \lambda x, \kappa_{2} \lambda x\right)\right|_{x=\tilde{x}}, \\
& G_{[\alpha \beta]}^{\mathrm{tw} 4(\mathrm{iiA}) \mathrm{b}}\left(\kappa_{1} \tilde{x}, \kappa_{2} \tilde{x}\right)=-\left.\frac{1}{2} x_{[\alpha} \partial_{\beta]} \square \int_{0}^{1} \mathrm{~d} \lambda\left(\frac{1-\lambda^{2}}{2 \lambda}+\lambda \ln \lambda\right) G\left(\kappa_{1} \lambda x, \kappa_{2} \lambda x\right)\right|_{x=\tilde{x}}, \\
& G_{[\alpha \beta]}^{\mathrm{tw} 5(\mathrm{iiA}) \mathrm{a}}\left(\kappa_{1} \tilde{x}, \kappa_{2} \tilde{x}\right)=-\left.x_{[\alpha}\left(\delta_{\beta]}^{\mu}(x \partial)-x^{\mu} \partial_{\beta]}\right) \square \int_{0}^{1} \mathrm{~d} \lambda \frac{(1-\lambda)^{2}}{4 \lambda} G_{\mu \bullet}\left(\kappa_{1} \lambda x, \kappa_{2} \lambda x\right)\right|_{x=\tilde{x}} .
\end{aligned}
$$

(b) Now, we determine the symmetric tensor operator $G_{(\alpha \beta)}^{\mathrm{tw} 3(\mathrm{iiA})}\left(\kappa_{1} \tilde{x}, \kappa_{2} \tilde{x}\right)$ on the light-cone. Putting eq. (3.28) into (3.31), after some lengthy but straightforward calculation (taking into account only the relevant terms of the $k$-summation and performing some partial integrations), we get the following result:

$$
\begin{aligned}
G_{(\alpha \beta)}^{\mathrm{tw} 3(\mathrm{iiA})}\left(\kappa_{1} \tilde{x}, \kappa_{2} \tilde{x}\right)= & \left.\int_{0}^{1} \mathrm{~d} \lambda \frac{1-\lambda^{2}}{2 \lambda}\left(\delta_{(\alpha}^{\mu}(x \partial)-x^{\mu} \partial_{(\alpha}\right) \partial_{\beta)} G_{\mu \bullet}\left(\lambda x, \kappa_{2} \lambda x\right)\right|_{x=\tilde{x}} \\
& -G_{(\alpha \beta)}^{>(\mathrm{iiA})}\left(\kappa_{1} \tilde{x}, \kappa_{2} \tilde{x}\right)
\end{aligned}
$$


with the higher twist contributions of the trace terms

$$
\begin{aligned}
G_{(\alpha \beta)}^{>(\mathrm{iiA})}\left(\kappa_{1} \tilde{x}, \kappa_{2} \tilde{x}\right)=\int_{0}^{1} \mathrm{~d} \lambda\left\{\left[\frac{1-\lambda^{2}}{2 \lambda} g_{\alpha \beta} \partial^{\mu}+\frac{1-\lambda}{2 \lambda} \delta_{(\alpha}^{\mu} x_{\beta} \square+(1-\lambda) x_{(\alpha} \partial_{\beta)} \partial^{\mu}\right.\right. \\
\left.-\frac{(1-\lambda)^{2}}{4 \lambda} x_{\alpha} x_{\beta} \partial^{\mu} \square\right] G_{\mu \bullet}\left(\kappa_{1} \lambda \tilde{x}, \kappa_{2} \lambda \tilde{x}\right)-\left[\frac{1}{2}\left(\frac{1-\lambda^{2}}{2 \lambda}+\lambda \ln \lambda\right) g_{\alpha \beta} \square\right. \\
+\left(\frac{1}{2}(1-\lambda)+\frac{1-\lambda^{2}}{4 \lambda}+\lambda \ln \lambda\right) x_{(\alpha} \partial_{\beta)} \square \\
\left.\left.+\frac{1}{4}\left(\frac{1-\lambda^{2}}{2 \lambda}-\frac{(1-\lambda)^{2}}{\lambda}+\lambda \ln \lambda\right) x_{\alpha} x_{\beta} \square^{2}\right] G\left(\kappa_{1} \lambda x, \kappa_{2} \lambda x\right)\right\}\left.\right|_{x=\tilde{x}} .
\end{aligned}
$$

It is obvious that eq. (3.40) contains scalar and vector operators. Again, using Young patterns (i) and (ii) and subtracting the trace terms, we can decompose the vector part appearing in eq. (3.40) into twist-4, twist-5 and twist-6 operators. This procedure is analogous to the twist decomposition of the (vector) quark operator $O_{\alpha}\left(\kappa_{1} \tilde{x}, \kappa_{2} \tilde{x}\right)$ in Part I. From the twist-4 and twist-5 vector part we have to subtract their trace terms being of twist -6 and add it to the other twist -6 scalar operator. Let us recall that the scalar twist-4 and twist- 6 operators are already traceless on the cone. In that manner we get the following decomposition:

$$
\begin{aligned}
G_{(\alpha \beta)}^{>(\mathrm{iiA})}\left(\kappa_{1} \tilde{x}, \kappa_{2} \tilde{x}\right)=G_{(\alpha \beta)}^{\mathrm{tw} 4(\mathrm{iiA}) \mathrm{a}}\left(\kappa_{1} \tilde{x}, \kappa_{2} \tilde{x}\right)+G_{(\alpha \beta)}^{\mathrm{tw} 4(\mathrm{iiA}) \mathrm{b}}\left(\kappa_{1} \tilde{x}, \kappa_{2} \tilde{x}\right)+G_{(\alpha \beta)}^{\mathrm{tw} 4(\mathrm{iiA}) \mathrm{c}}\left(\kappa_{1} \tilde{x}, \kappa_{2} \tilde{x}\right) \\
+G_{(\alpha \beta)}^{\mathrm{tw} 4(\mathrm{iiA}) \mathrm{d} 1}\left(\kappa_{1} \tilde{x}, \kappa_{2} \tilde{x}\right)+G_{(\alpha \beta)}^{\mathrm{tw} 4(\mathrm{iiA}) \mathrm{d} 2}\left(\kappa_{1} \tilde{x}, \kappa_{2} \tilde{x}\right)+G_{(\alpha \beta)}^{\mathrm{tw} 4(\mathrm{iiA}) \mathrm{e}}\left(\kappa_{1} \tilde{x}, \kappa_{2} \tilde{x}\right) \\
+G_{(\alpha \beta)}^{\mathrm{tw} 5(\mathrm{iiA}) \mathrm{d}}\left(\kappa_{1} \tilde{x}, \kappa_{2} \tilde{x}\right)+G_{(\alpha \beta)}^{\mathrm{tw} 6(\mathrm{iiA}) \mathrm{a}}\left(\kappa_{1} \tilde{x}, \kappa_{2} \tilde{x}\right)+G_{(\alpha \beta)}^{\mathrm{tw} 6(\mathrm{iiA}) \mathrm{b}}\left(\kappa_{1} \tilde{x}, \kappa_{2} \tilde{x}\right) \\
+G_{(\alpha \beta)}^{\mathrm{tw} 6(\mathrm{iiA}) \mathrm{c}}\left(\kappa_{1} \tilde{x}, \kappa_{2} \tilde{x}\right)+G_{(\alpha \beta)}^{\mathrm{tw} 6(\mathrm{iiA}) \mathrm{d} 1}\left(\kappa_{1} \tilde{x}, \kappa_{2} \tilde{x}\right)+G_{(\alpha \beta)}^{\mathrm{tw} 6(\mathrm{iiA}) \mathrm{d} 2}\left(\kappa_{1} \tilde{x}, \kappa_{2} \tilde{x}\right) \\
+G_{(\alpha \beta)}^{\mathrm{tw} 6(\mathrm{iiA}) \mathrm{d} 3}\left(\kappa_{1} \tilde{x}, \kappa_{2} \tilde{x}\right)+G_{(\alpha \beta)}^{\mathrm{tw} 6(\mathrm{iiA}) \mathrm{d} 4}\left(\kappa_{1} \tilde{x}, \kappa_{2} \tilde{x}\right)+G_{(\alpha \beta)}^{\mathrm{tw} 6(\mathrm{iiA}) \mathrm{e}}\left(\kappa_{1} \tilde{x}, \kappa_{2} \tilde{x}\right),
\end{aligned}
$$

with

$$
\begin{aligned}
G_{(\alpha \beta)}^{\mathrm{tw} 4(\mathrm{iiA}) \mathrm{a}}\left(\kappa_{1} \tilde{x}, \kappa_{2} \tilde{x}\right)= & -\left.\frac{1}{2} g_{\alpha \beta} \square \int_{0}^{1} \mathrm{~d} \lambda\left(\frac{1-\lambda^{2}}{2 \lambda}+\lambda \ln \lambda\right) G\left(\kappa_{1} \lambda x, \kappa_{2} \lambda x\right)\right|_{x=\tilde{x}}, \\
G_{(\alpha \beta)}^{\mathrm{tw} 4(\mathrm{iiA}) \mathrm{b}}\left(\kappa_{1} \tilde{x}, \kappa_{2} \tilde{x}\right)= & -\frac{1}{2} x_{(\alpha} \partial_{\beta} \square \int_{0}^{1} \mathrm{~d} \lambda\left((1-\lambda)+\frac{1-\lambda^{2}}{2 \lambda}\right. \\
& +2 \lambda \ln \lambda)\left.G\left(\kappa_{1} \lambda x, \kappa_{2} \lambda x\right)\right|_{x=\tilde{x}}-G_{(\alpha \beta)}^{\mathrm{tw} 6(\mathrm{iiA}) \mathrm{b}}\left(\kappa_{1} \tilde{x}, \kappa_{2} \tilde{x}\right), \\
G_{(\alpha \beta)}^{\mathrm{tw} 4(\mathrm{iiA}) \mathrm{d} 1}\left(\kappa_{1} \tilde{x}, \kappa_{2} \tilde{x}\right)= & -\left.\frac{1}{2} x_{(\alpha} \partial_{\beta} \square \int_{0}^{1} \mathrm{~d} \lambda\left(\frac{1-\lambda}{\lambda}+\frac{\ln \lambda}{\lambda}\right) G\left(\kappa_{1} \lambda x, \kappa_{2} \lambda x\right)\right|_{x=\tilde{x}} \\
& -G_{(\alpha \beta)}^{\mathrm{tw} 6(\mathrm{iiA}) \mathrm{d} 1}\left(\kappa_{1} \tilde{x}, \kappa_{2} \tilde{x}\right),
\end{aligned}
$$


and

$$
\begin{gathered}
G_{(\alpha \beta)}^{\mathrm{tw} 6(\mathrm{iiA}) \mathrm{a}}\left(\kappa_{1} \tilde{x}, \kappa_{2} \tilde{x}\right)=\left.\frac{1}{4} x_{\alpha} x_{\beta} \square^{2} \int_{0}^{1} \mathrm{~d} \lambda\left(\frac{(1-\lambda)^{2}}{\lambda}-\frac{1-\lambda^{2}}{2 \lambda}-\lambda \ln \lambda\right) G\left(\kappa_{1} \lambda x, \kappa_{2} \lambda x\right)\right|_{x=\tilde{x}}, \\
G_{(\alpha \beta)}^{\mathrm{tw} 6(\mathrm{iiA}) \mathrm{b}}\left(\kappa_{1} \tilde{x}, \kappa_{2} \tilde{x}\right)=-\frac{1}{4} x_{\alpha} x_{\beta} \square^{2} \int_{0}^{1} \mathrm{~d} \lambda\left(\frac{(1-\lambda)^{2}}{2 \lambda}-\frac{3}{2} \frac{1-\lambda^{2}}{2 \lambda}\right. \\
\left.-\lambda \ln \lambda-\frac{\ln \lambda}{2 \lambda}\right)\left.G\left(\kappa_{1} \lambda x, \kappa_{2} \lambda x\right)\right|_{x=\tilde{x}}, \\
G_{(\alpha \beta)}^{\mathrm{tw} 6(\mathrm{iiA}) \mathrm{d} 1}\left(\kappa_{1} \tilde{x}, \kappa_{2} \tilde{x}\right)=\left.\frac{1}{4} x_{\alpha} x_{\beta} \square^{2} \int_{0}^{1} \mathrm{~d} \lambda\left(\frac{1-\lambda}{\lambda}+\frac{\ln \lambda}{\lambda}+\frac{\ln ^{2} \lambda}{2 \lambda}\right) G\left(\kappa_{1} \lambda x, \kappa_{2} \lambda x\right)\right|_{x=\tilde{x}}, \\
G_{(\alpha \beta)}^{\mathrm{tw} 6(\mathrm{iiA}) \mathrm{d} 3}\left(\kappa_{1} \tilde{x}, \kappa_{2} \tilde{x}\right)=-\left.\frac{1}{2} x_{\alpha} x_{\beta} \square^{2} \int_{0}^{1} \mathrm{~d} \lambda\left(\frac{1-\lambda}{\lambda}+\frac{\ln \lambda}{\lambda}+\frac{\ln ^{2} \lambda}{2 \lambda}\right) G\left(\kappa_{1} \lambda x, \kappa_{2} \lambda x\right)\right|_{x=\tilde{x}},
\end{gathered}
$$

being related to the scalar operator $G\left(\kappa_{1} \tilde{x}, \kappa_{2} \tilde{x}\right)$, as well as

$$
\begin{aligned}
G_{(\alpha \beta)}^{\mathrm{tw} 4(\mathrm{iiA}) \mathrm{c}}\left(\kappa_{1} \tilde{x}, \kappa_{2} \tilde{x}\right)= & \left.\frac{1}{2} g_{\alpha \beta} \partial^{\mu} \int_{0}^{1} \mathrm{~d} \lambda \frac{1-\lambda^{2}}{\lambda} G_{\mu \bullet}\left(\kappa_{1} \lambda x, \kappa_{2} \lambda x\right)\right|_{x=\tilde{x}} \\
G_{(\alpha \beta)}^{\mathrm{tw} 4(\mathrm{iiA}) \mathrm{d} 2}\left(\kappa_{1} \tilde{x}, \kappa_{2} \tilde{x}\right)= & \left.x_{(\alpha} \partial_{\beta)} \partial^{\mu} \int_{0}^{1} \mathrm{~d} \lambda\left(\frac{1-\lambda}{\lambda}+\frac{\ln \lambda}{\lambda}\right) G_{\mu \bullet}\left(\kappa_{1} \lambda x, \kappa_{2} \lambda x\right)\right|_{x=\tilde{x}} \\
& -G_{(\alpha \beta)}^{\mathrm{tw} 6(\mathrm{iiA}) \mathrm{d} 2}\left(\kappa_{1} \tilde{x}, \kappa_{2} \tilde{x}\right) \\
G_{(\alpha \beta)}^{\mathrm{tw} 4(\mathrm{iiA}) \mathrm{e}}\left(\kappa_{1} \tilde{x}, \kappa_{2} \tilde{x}\right)= & \left.x_{(\alpha} \partial_{\beta)} \partial^{\mu} \int_{0}^{1} \mathrm{~d} \lambda(1-\lambda) G_{\mu \bullet}\left(\kappa_{1} \lambda x, \kappa_{2} \lambda x\right)\right|_{x=\tilde{x}} \\
& -G_{(\alpha \beta)}^{\mathrm{tw} 6(\mathrm{iiA}) \mathrm{e}}\left(\kappa_{1} \tilde{x}, \kappa_{2} \tilde{x}\right)
\end{aligned}
$$

and

$$
\begin{aligned}
G_{(\alpha \beta)}^{\mathrm{tw} 5(\mathrm{iiA})}\left(\kappa_{1} \tilde{x}, \kappa_{2} \tilde{x}\right)= & -\frac{1}{2} x_{(\alpha}\left(\delta_{\beta)}^{\mu}(x \partial)-x^{\mu} \partial_{\beta)}\right) \square \\
& \times\left.\int_{0}^{1} \mathrm{~d} \lambda\left(\frac{1-\lambda}{\lambda}+\frac{\ln \lambda}{\lambda}\right) G_{\mu \bullet}\left(\kappa_{1} \lambda x, \kappa_{2} \lambda x\right)\right|_{x=\tilde{x}}, \\
& -G_{(\alpha \beta)}^{\mathrm{tw} 6(\mathrm{iiA}) \mathrm{d} 3}\left(\kappa_{1} \tilde{x}, \kappa_{2} \tilde{x}\right)-G_{(\alpha \beta)}^{\mathrm{tw} 6(\mathrm{iiA}) \mathrm{d} 4}\left(\kappa_{1} \tilde{x}, \kappa_{2} \tilde{x}\right),
\end{aligned}
$$

and

$$
\begin{aligned}
G_{(\alpha \beta)}^{\mathrm{tw} 6(\mathrm{iiA}) \mathrm{c}}\left(\kappa_{1} \tilde{x}, \kappa_{2} \tilde{x}\right) & =-\left.\frac{1}{4} x_{\alpha} x_{\beta} \square \partial^{\mu} \int_{0}^{1} \mathrm{~d} \lambda \frac{(1-\lambda)^{2}}{\lambda} G_{\mu \bullet}\left(\kappa_{1} \lambda x, \kappa_{2} \lambda x\right)\right|_{x=\tilde{x}}, \\
G_{(\alpha \beta)}^{\mathrm{tw} 6(\mathrm{iiA}) \mathrm{d} 2}\left(\kappa_{1} \tilde{x}, \kappa_{2} \tilde{x}\right) & =-\left.\frac{1}{2} x_{\alpha} x_{\beta} \square \partial^{\mu} \int_{0}^{1} \mathrm{~d} \lambda\left(\frac{1-\lambda}{\lambda}+\frac{\ln \lambda}{\lambda}+\frac{\ln ^{2} \lambda}{2 \lambda}\right) G_{\mu \bullet}\left(\kappa_{1} \lambda x, \kappa_{2} \lambda x\right)\right|_{x=\tilde{x}}, \\
G_{(\alpha \beta)}^{\mathrm{tw} 6(\mathrm{iiA}) \mathrm{d} 4}\left(\kappa_{1} \tilde{x}, \kappa_{2} \tilde{x}\right) & =-\left.\frac{1}{2} x_{\alpha} x_{\beta} \square \partial^{\mu} \int_{0}^{1} \mathrm{~d} \lambda\left(\frac{1-\lambda}{\lambda}+\frac{\ln \lambda}{\lambda}\right) G_{\mu \bullet}\left(\kappa_{1} \lambda x, \kappa_{2} \lambda x\right)\right|_{x=\tilde{x}}, \\
G_{(\alpha \beta)}^{\mathrm{tw} 6(\mathrm{iiA}) \mathrm{e}}\left(\kappa_{1} \tilde{x}, \kappa_{2} \tilde{x}\right) & =\left.\frac{1}{4} x_{\alpha} x_{\beta} \square \partial^{\mu} \int_{0}^{1} \mathrm{~d} \lambda \frac{(1-\lambda)^{2}}{\lambda} G_{\mu \bullet}\left(\kappa_{1} \lambda x, \kappa_{2} \lambda x\right)\right|_{x=\tilde{x}},
\end{aligned}
$$

being related to the vector operator $G_{\mu \bullet}\left(\kappa_{1} \tilde{x}, \kappa_{2} \tilde{x}\right)$. 


\subsubsection{Contributions of Young tableau B}

Let us now consider the other Young tableau which is obtained from the former one by exchanging $\alpha$ and $\beta$ :

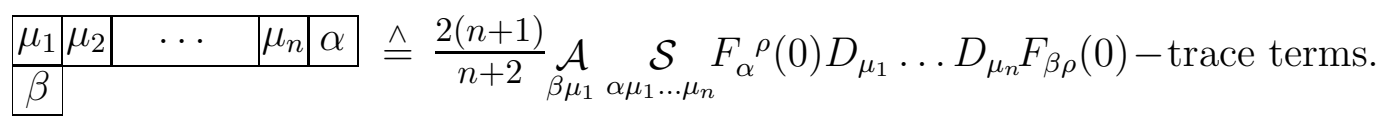

This symmetry behaviour will be denoted by (iiB). The corresponding nonlocal twist-3 operator which we obtain after analogous calculations is (with selfexplaining denotations):

$$
G_{\alpha \beta}^{\mathrm{tw} 3(\mathrm{iiB})}(0, \kappa x)=\int_{0}^{1} \mathrm{~d} \lambda \frac{1-\lambda^{2}}{2 \lambda}\left(\delta_{\beta}^{\mu}(x \partial)-x^{\mu} \partial_{\beta}\right) \partial_{\alpha} \stackrel{\circ}{G}_{\bullet \mu}(0, \kappa \lambda x) .
$$

Its decomposition into symmetric and antisymmetric part yields

$$
G_{\alpha \beta}^{\mathrm{tw} 3(\mathrm{iiB})}(0, \kappa x)=G_{[\alpha \beta]}^{\mathrm{tw} 3(\mathrm{iiB})}(0, \kappa x)+G_{(\alpha \beta)}^{\mathrm{tw} 3(\mathrm{iiB})}(0, \kappa x)
$$

with

$$
\begin{aligned}
& G_{[\alpha \beta]}^{\mathrm{tw} 3(\mathrm{iiB})}(0, \kappa x)=\int_{0}^{1} \mathrm{~d} \lambda \lambda \delta_{[\beta}^{\mu} \partial_{\alpha]} \stackrel{\circ}{G}_{\bullet}(0, \kappa \lambda x), \\
& G_{(\alpha \beta)}^{\mathrm{tw} 3(\mathrm{iiB})}(0, \kappa x)=\int_{0}^{1} \mathrm{~d} \lambda \frac{1-\lambda^{2}}{2 \lambda}\left(\delta_{(\alpha}^{\mu}(x \partial)-x^{\mu} \partial_{(\alpha)}\right) \partial_{\beta)} \stackrel{\circ}{G}_{\bullet \mu}(0, \kappa \lambda x) .
\end{aligned}
$$

The projection onto the light-cone and the calculation of the higher twist operators contained in the trace terms is completely analogous to case (A) and should be omitted here.

\subsubsection{Construction of the complete twist-3 tensor operators on the light-cone}

In order to obtain the complete twist- 3 operator it is necessary to add both twist3 operators (3.29) and (3.43) resulting from the Young patterns (iiA) and (iiB). Since no further Young pattern contributes to twist-3 operators we omit the index (ii). After projection onto the light-cone the final result is

$$
G_{\alpha \beta}^{\mathrm{tw} 3}\left(\kappa_{1} \tilde{x}, \kappa_{2} \tilde{x}\right)=G_{[\alpha \beta]}^{\mathrm{tw} 3}\left(\kappa_{1} \tilde{x}, \kappa_{2} \tilde{x}\right)+G_{(\alpha \beta)}^{\mathrm{tw} 3}\left(\kappa_{1} \tilde{x}, \kappa_{2} \tilde{x}\right),
$$

with the antisymmetric twist-3 light-cone tensor operator

$$
\begin{aligned}
G_{[\alpha \beta]}^{\mathrm{tw} 3}\left(\kappa_{1} \tilde{x}, \kappa_{2} \tilde{x}\right)= & \left.2 \int_{0}^{1} \mathrm{~d} \lambda \lambda \partial_{[\alpha} G_{\beta]}^{-}\left(\kappa_{1} \lambda x, \kappa_{2} \lambda x\right)\right|_{x=\tilde{x}} \\
& -G_{[\alpha \beta]}^{\mathrm{tw} 4(\mathrm{ii})}\left(\kappa_{1} \tilde{x}, \kappa_{2} \tilde{x}\right)-G_{[\alpha \beta]}^{\mathrm{tw} 5(\mathrm{ii})}\left(\kappa_{1} \tilde{x}, \kappa_{2} \tilde{x}\right),
\end{aligned}
$$

\footnotetext{
${ }^{6}$ Here, we used the abbreviation $G_{\mu}^{-} \equiv x^{\nu} G_{[\mu \nu]}$ instead of $G_{[\mu \bullet]}$ in order not to come into conflict with the antisymmetrization of the indices $\alpha$ and $\beta$.
} 
where

$$
\begin{aligned}
& G_{[\alpha \beta]}^{\mathrm{tw} 4(\mathrm{ii})}\left(\kappa_{1} \tilde{x}, \kappa_{2} \tilde{x}\right)=\left.x_{[\alpha} \partial_{\beta]} \partial^{\mu} \int_{0}^{1} \mathrm{~d} \lambda \frac{1-\lambda^{2}}{\lambda} G_{\mu}^{-}\left(\kappa_{1} \lambda x, \kappa_{2} \lambda x\right)\right|_{x=\tilde{x}}, \\
& G_{[\alpha \beta]}^{\mathrm{tw} 5(\mathrm{ii})}\left(\kappa_{1} \tilde{x}, \kappa_{2} \tilde{x}\right)=-\left.x_{[\alpha}\left(\delta_{\beta]}^{\mu}(x \partial)-x^{\mu} \partial_{\beta]}\right) \square \int_{0}^{1} \mathrm{~d} \lambda \frac{(1-\lambda)^{2}}{2 \lambda} G_{\mu}^{-}\left(\kappa_{1} \lambda x, \kappa_{2} \lambda x\right)\right|_{x=\tilde{x}} .
\end{aligned}
$$

The local twist -4 operators are contained in the tensor space $\mathbf{T}\left(\frac{n}{2}, \frac{n}{2}\right)$ and the local twist -5 operators are contained in the tensor space $\mathbf{T}\left(\frac{n}{2}, \frac{n-2}{2}\right) \oplus \mathbf{T}\left(\frac{n-2}{2}, \frac{n}{2}\right)$.

In turn, let us remark that the bilocal light-ray operator $G_{[\alpha \beta]}^{\mathrm{tw} 3}\left(\kappa_{1} \tilde{x}, \kappa_{2} \tilde{x}\right)$ is the same as the antisymmetric tensor operator which obtains from the Young tableau

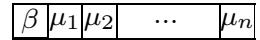

. That tableau has been used in Part I for the computation of $M_{[\alpha \beta]}^{\mathrm{tw} 2}\left(\kappa_{1} \tilde{x}, \kappa_{2} \tilde{x}\right)$. Furthermore, let us note that the local operators contained in $M_{[\alpha \beta]}^{\mathrm{tw} 2}\left(\kappa_{1} \tilde{x}, \kappa_{2} \tilde{x}\right)$ and in $G_{[\alpha \beta]}^{\mathrm{tw} 3}\left(\kappa_{1} \tilde{x}, \kappa_{2} \tilde{x}\right)$ are in complete agreement with the local operators on the light-cone determined by Dobrev and Ganchev [17] by means of the interior derivative.

From the above point of view our result for the antisymmetric part of twist-3 operator (3.46) could be obtained much easier. However, the symmetric twist-3 light-cone tensor operator is much more involved. It is given by

$$
\begin{aligned}
G_{(\alpha \beta)}^{\mathrm{tw} 3}\left(\kappa_{1} \tilde{x}, \kappa_{2} \tilde{x}\right)= & \left.\int_{0}^{1} \mathrm{~d} \lambda \frac{1-\lambda^{2}}{2 \lambda}\left(\delta_{(\alpha}^{\mu}(x \partial)-x^{\mu} \partial_{(\alpha}\right) \partial_{\beta)} G_{\mu}^{+}\left(\kappa_{1} \lambda x, \kappa_{2} \lambda x\right)\right|_{x=\tilde{x}} \\
& -G_{(\alpha \beta)}^{>(\mathrm{ii})}\left(\kappa_{1} \tilde{x}, \kappa_{2} \tilde{x}\right),
\end{aligned}
$$

where $G_{(\alpha \beta)}^{>(\mathrm{ii})}\left(\kappa_{1} \tilde{x}, \kappa_{2} \tilde{x}\right)$ includes all the trace terms having higher twist; these operators are given by

$$
\begin{aligned}
G_{(\alpha \beta)}^{\mathrm{tw} 4(\mathrm{ii)}) \mathrm{a}}\left(\kappa_{1} \tilde{x}, \kappa_{2} \tilde{x}\right)= & -\left.g_{\alpha \beta} \square \int_{0}^{1} \mathrm{~d} \lambda\left(\frac{1-\lambda^{2}}{2 \lambda}+\lambda \ln \lambda\right) G\left(\kappa_{1} \lambda x, \kappa_{2} \lambda x\right)\right|_{x=\tilde{x}}, \\
G_{(\alpha \beta)}^{\mathrm{tw} 4(\mathrm{ii}) \mathrm{b}}\left(\kappa_{1} \tilde{x}, \kappa_{2} \tilde{x}\right)= & -\left.x_{(\alpha} \partial_{\beta)} \square \int_{0}^{1} \mathrm{~d} \lambda\left((1-\lambda)+\frac{1-\lambda^{2}}{2 \lambda}+2 \lambda \ln \lambda\right) G\left(\kappa_{1} \lambda x, \kappa_{2} \lambda x\right)\right|_{x=\tilde{x}} \\
& -G_{(\alpha \beta)}^{\mathrm{tw} 6(\mathrm{ii}) \mathrm{b}}\left(\kappa_{1} \tilde{x}, \kappa_{2} \tilde{x}\right), \\
G_{(\alpha \beta)}^{\mathrm{tw} 4(\mathrm{ii}) \mathrm{d} 1}\left(\kappa_{1} \tilde{x}, \kappa_{2} \tilde{x}\right)= & -\left.x_{(\alpha} \partial_{\beta)} \square \int_{0}^{1} \mathrm{~d} \lambda\left(\frac{1-\lambda}{\lambda}+\frac{\ln \lambda}{\lambda}\right) G\left(\kappa_{1} \lambda x, \kappa_{2} \lambda x\right)\right|_{x=\tilde{x}} \\
& -G_{(\alpha \beta)}^{\mathrm{tw} 6(\mathrm{ii}) \mathrm{d} 1}\left(\kappa_{1} \tilde{x}, \kappa_{2} \tilde{x}\right),
\end{aligned}
$$

\footnotetext{
${ }^{7}$ Similarly, we use the notation $G_{\mu}^{+}$instead of $G_{(\mu \bullet)}$.
} 
and

$$
\begin{gathered}
G_{(\alpha \beta)}^{\mathrm{tw} 6(\mathrm{ii)}) \mathrm{a}}\left(\kappa_{1} \tilde{x}, \kappa_{2} \tilde{x}\right)=\left.\frac{1}{2} x_{\alpha} x_{\beta} \square^{2} \int_{0}^{1} \mathrm{~d} \lambda\left(\frac{(1-\lambda)^{2}}{\lambda}-\frac{1-\lambda^{2}}{2 \lambda}-\lambda \ln \lambda\right) G\left(\kappa_{1} \lambda x, \kappa_{2} \lambda x\right)\right|_{x=\tilde{x}}, \\
G_{(\alpha \beta)}^{\mathrm{tw} 6(\mathrm{ii)b}}\left(\kappa_{1} \tilde{x}, \kappa_{2} \tilde{x}\right)=-\frac{1}{2} x_{\alpha} x_{\beta} \square^{2} \int_{0}^{1} \mathrm{~d} \lambda\left(\frac{(1-\lambda)^{2}}{2 \lambda}-\frac{3}{2} \frac{1-\lambda^{2}}{2 \lambda}\right. \\
\left.-\lambda \ln \lambda-\frac{\ln \lambda}{2 \lambda}\right)\left.G\left(\kappa_{1} \lambda x, \kappa_{2} \lambda x\right)\right|_{x=\tilde{x}}, \\
G_{(\alpha \beta)}^{\mathrm{tw} 6(\mathrm{ii}) \mathrm{d} 1}\left(\kappa_{1} \tilde{x}, \kappa_{2} \tilde{x}\right)=\left.\frac{1}{2} x_{\alpha} x_{\beta} \square^{2} \int_{0}^{1} \mathrm{~d} \lambda\left(\frac{1-\lambda}{\lambda}+\frac{\ln \lambda}{\lambda}+\frac{\ln ^{2} \lambda}{2 \lambda}\right) G\left(\kappa_{1} \lambda x, \kappa_{2} \lambda x\right)\right|_{x=\tilde{x}}, \\
G_{(\alpha \beta)}^{\mathrm{tw} 6(\mathrm{ii}) \mathrm{d} 3}\left(\kappa_{1} \tilde{x}, \kappa_{2} \tilde{x}\right)=-\left.x_{\alpha} x_{\beta} \square^{2} \int_{0}^{1} \mathrm{~d} \lambda\left(\frac{1-\lambda}{\lambda}+\frac{\ln \lambda}{\lambda}+\frac{\ln ^{2} \lambda}{2 \lambda}\right) G\left(\kappa_{1} \lambda x, \kappa_{2} \lambda x\right)\right|_{x=\tilde{x}},
\end{gathered}
$$

being related to the scalar operator $G\left(\kappa_{1} x, \kappa_{2} x\right)$, as well as

$$
\begin{aligned}
G_{(\alpha \beta)}^{\mathrm{tw} 4(\mathrm{ii)} \mathrm{c}}\left(\kappa_{1} \tilde{x}, \kappa_{2} \tilde{x}\right)= & \left.g_{\alpha \beta} \partial^{\mu} \int_{0}^{1} \mathrm{~d} \lambda \frac{1-\lambda^{2}}{\lambda} G_{\mu}^{+}\left(\kappa_{1} \lambda x, \kappa_{2} \lambda x\right)\right|_{x=\tilde{x}}, \\
G_{(\alpha \beta)}^{\mathrm{tw} 4(\mathrm{ii}) \mathrm{d} 2}\left(\kappa_{1} \tilde{x}, \kappa_{2} \tilde{x}\right)= & \left.2 x_{(\alpha} \partial_{\beta)} \partial^{\mu} \int_{0}^{1} \mathrm{~d} \lambda\left(\frac{1-\lambda}{\lambda}+\frac{\ln \lambda}{\lambda}\right) G_{\mu}^{+}\left(\kappa_{1} \lambda x, \kappa_{2} \lambda x\right)\right|_{x=\tilde{x}} \\
& -G_{(\alpha \beta)}^{\mathrm{tw} 6(\mathrm{ii}) \mathrm{d} 2}\left(\kappa_{1} \tilde{x}, \kappa_{2} \tilde{x}\right), \\
G_{(\alpha \beta)}^{\mathrm{tw} 4(\mathrm{ii}) \mathrm{e}}\left(\kappa_{1} \tilde{x}, \kappa_{2} \tilde{x}\right)= & \left.2 x_{(\alpha} \partial_{\beta)} \partial^{\mu} \int_{0}^{1} \mathrm{~d} \lambda(1-\lambda) G_{\mu}^{+}\left(\kappa_{1} \lambda x, \kappa_{2} \lambda x\right)\right|_{x=\tilde{x}} \\
& -G_{(\alpha \beta)}^{\mathrm{tw} 6(\mathrm{ii}) \mathrm{e}}\left(\kappa_{1} \tilde{x}, \kappa_{2} \tilde{x}\right),
\end{aligned}
$$

and

$$
\begin{aligned}
G_{(\alpha \beta)}^{\mathrm{tw} 5(\mathrm{ii})}\left(\kappa_{1} \tilde{x}, \kappa_{2} \tilde{x}\right)= & -x_{(\alpha}\left(\delta_{\beta)}^{\mu}(x \partial)-x^{\mu} \partial_{\beta)}\right) \square \\
& \times\left.\int_{0}^{1} \mathrm{~d} \lambda\left(\frac{1-\lambda}{\lambda}+\frac{\ln \lambda}{\lambda}\right) G_{\mu}^{+}\left(\kappa_{1} \lambda x, \kappa_{2} \lambda x\right)\right|_{x=\tilde{x}}, \\
& -G_{(\alpha \beta)}^{\mathrm{tw} 6(\mathrm{ii}) \mathrm{d} 3}\left(\kappa_{1} \tilde{x}, \kappa_{2} \tilde{x}\right)-G_{(\alpha \beta)}^{\mathrm{tw} 6(\mathrm{ii}) \mathrm{d} 4}\left(\kappa_{1} \tilde{x}, \kappa_{2} \tilde{x}\right),
\end{aligned}
$$

and

$$
\begin{aligned}
G_{(\alpha \beta)}^{\mathrm{tw} 6(\mathrm{ii}) \mathrm{c}}\left(\kappa_{1} \tilde{x}, \kappa_{2} \tilde{x}\right) & =-\left.\frac{1}{2} x_{\alpha} x_{\beta} \square \partial^{\mu} \int_{0}^{1} \mathrm{~d} \lambda \frac{(1-\lambda)^{2}}{\lambda} G_{\mu}^{+}\left(\kappa_{1} \lambda x, \kappa_{2} \lambda x\right)\right|_{x=\tilde{x}}, \\
G_{(\alpha \beta)}^{\mathrm{tw} 6(\mathrm{ii}) \mathrm{d} 2}\left(\kappa_{1} \tilde{x}, \kappa_{2} \tilde{x}\right) & =-\left.x_{\alpha} x_{\beta} \square \partial^{\mu} \int_{0}^{1} \mathrm{~d} \lambda\left(\frac{1-\lambda}{\lambda}+\frac{\ln \lambda}{\lambda}+\frac{\ln ^{2} \lambda}{2 \lambda}\right) G_{\mu}^{+}\left(\kappa_{1} \lambda x, \kappa_{2} \lambda x\right)\right|_{x=\tilde{x}}, \\
G_{(\alpha \beta)}^{\mathrm{tw} 6(\mathrm{ii}) \mathrm{d} 4}\left(\kappa_{1} \tilde{x}, \kappa_{2} \tilde{x}\right) & =-\left.x_{\alpha} x_{\beta} \square \partial^{\mu} \int_{0}^{1} \mathrm{~d} \lambda\left(\frac{1-\lambda}{\lambda}+\frac{\ln \lambda}{\lambda}\right) G_{\mu}^{+}\left(\kappa_{1} \lambda x, \kappa_{2} \lambda x\right)\right|_{x=\tilde{x}}, \\
G_{(\alpha \beta)}^{\mathrm{tw} 6(\mathrm{ii}) \mathrm{e}}\left(\kappa_{1} \tilde{x}, \kappa_{2} \tilde{x}\right) & =\left.\frac{1}{2} x_{\alpha} x_{\beta} \square \partial^{\mu} \int_{0}^{1} \mathrm{~d} \lambda \frac{(1-\lambda)^{2}}{\lambda} G_{\mu}^{+}\left(\kappa_{1} \lambda x, \kappa_{2} \lambda x\right)\right|_{x=\tilde{x}},
\end{aligned}
$$

being related to the (symmetric) vector operator $G_{\mu}^{+}\left(\kappa_{1} x, \kappa_{2} x\right)$. 


\subsubsection{Determination of complete light-cone vector operators}

Now we are able to determine the twist-3 vector operator resulting from the tensor operator (3.46) by multiplying with $x^{\beta}$. Because neither symmetry type (iii) nor (iv) may contribute to the vector operator this will be the final expression. The twist-3 light-cone vector operator consists of two parts, one originating from the symmetric and the other from the antisymmetric tensor operator,

$$
G_{\alpha}^{\mathrm{tw} 3}\left(\kappa_{1} \tilde{x}, \kappa_{2} \tilde{x}\right)=G_{(\alpha \bullet)}^{\mathrm{tw} 3}\left(\kappa_{1} \tilde{x}, \kappa_{2} \tilde{x}\right)+G_{[\alpha \bullet}^{\mathrm{tw} 3}\left(\kappa_{1} \tilde{x}, \kappa_{2} \tilde{x}\right),
$$

where

$$
\begin{aligned}
& G_{(\alpha \bullet)}^{\mathrm{tw} 3}\left(\kappa_{1} \tilde{x}, \kappa_{2} \tilde{x}\right)=\left.\int_{0}^{1} \mathrm{~d} \lambda \lambda\left[\delta_{\alpha}^{\mu}(x \partial)-x^{\mu} \partial_{\alpha}-x_{\alpha}\left(\partial^{\mu}+\ln \lambda \square x^{\mu}\right)\right] G_{\mu}^{+}\left(\kappa_{1} \lambda x, \kappa_{2} \lambda x\right)\right|_{x=\tilde{x}} \\
& G_{[\alpha \bullet \bullet}^{\mathrm{tw} 3}\left(\kappa_{1} \tilde{x}, \kappa_{2} \tilde{x}\right)=\left.\int_{0}^{1} \mathrm{~d} \lambda \lambda\left[\delta_{\alpha}^{\mu}(x \partial)-x^{\mu} \partial_{\alpha}-x_{\alpha} \partial^{\mu}\right] G_{\mu}^{-}\left(\kappa_{1} \lambda x, \kappa_{2} \lambda x\right)\right|_{x=\tilde{x}}
\end{aligned}
$$

and the twist-4 vector operator which is contained in the trace terms of that twist -3 vector operator is given by

$$
G_{\alpha}^{\mathrm{tw} 4(\mathrm{ii})}\left(\kappa_{1} \tilde{x}, \kappa_{2} \tilde{x}\right)=G_{(\alpha \bullet)}^{\mathrm{tw} 4(\mathrm{ii})}\left(\kappa_{1} \tilde{x}, \kappa_{2} \tilde{x}\right)+G_{\left[\boldsymbol{\bullet}_{\bullet}\right]}^{\mathrm{tw} 4}\left(\kappa_{1} \tilde{x}, \kappa_{2} \tilde{x}\right),
$$

where

$$
\begin{aligned}
G_{(\alpha \bullet)}^{\mathrm{tw} 4(\mathrm{ii})}\left(\kappa_{1} \tilde{x}, \kappa_{2} \tilde{x}\right) & =\left.x_{\alpha} \int_{0}^{1} \mathrm{~d} \lambda \lambda\left[\partial^{\mu}+(\ln \lambda) \square x^{\mu}\right] G_{\mu}^{+}\left(\kappa_{1} \lambda x, \kappa_{2} \lambda x\right)\right|_{x=\tilde{x}}, \\
G_{[\alpha \bullet]}^{\mathrm{tw} 4}\left(\kappa_{1} \tilde{x}, \kappa_{2} \tilde{x}\right) & =\left.x_{\alpha} \int_{0}^{1} \mathrm{~d} \lambda \lambda \partial^{\mu} G_{\mu}^{-}\left(\kappa_{1} \lambda x, \kappa_{2} \lambda x\right)\right|_{x=\tilde{x}} .
\end{aligned}
$$

The antisymmetric part of the twist -4 vector operator is already complete. The complete twist-4 light-cone vector operator is given by

$$
\begin{aligned}
G_{\alpha}^{\mathrm{tw} 4}\left(\kappa_{1} \tilde{x}, \kappa_{2} \tilde{x}\right) & =G_{(\alpha \bullet \bullet}^{\mathrm{tw} 4}\left(\kappa_{1} \tilde{x}, \kappa_{2} \tilde{x}\right)+G_{\left[\boldsymbol{\alpha}_{\bullet}\right]}^{\mathrm{tw} 4}\left(\kappa_{1} \tilde{x}, \kappa_{2} \tilde{x}\right) \\
& =\left.x_{\alpha} \int_{0}^{1} \mathrm{~d} \lambda \lambda\left[\partial^{\mu}+\frac{\ln \lambda}{2} \square x^{\mu}\right] G_{\mu}\left(\kappa_{1} \lambda x, \kappa_{2} \lambda x\right)\right|_{x=\tilde{x}},
\end{aligned}
$$

where the symmetric twist- 4 vector operator obtains by adding together expressions (3.25) and (3.54),

$$
\begin{aligned}
G_{(\boldsymbol{\bullet})}^{\mathrm{tw} 4}\left(\kappa_{1} \tilde{x}, \kappa_{2} \tilde{x}\right) & =G_{(\alpha \bullet)}^{\mathrm{tw} 4(\mathrm{i})}\left(\kappa_{1} \tilde{x}, \kappa_{2} \tilde{x}\right)+G_{(\alpha \bullet)}^{\mathrm{tw} 4(\mathrm{ii})}\left(\kappa_{1} \tilde{x}, \kappa_{2} \tilde{x}\right) \\
& =\left.x_{\alpha} \int_{0}^{1} \mathrm{~d} \lambda \lambda\left[\partial^{\mu}+\frac{\ln \lambda}{2} \square x^{\mu}\right] G_{\mu}^{+}\left(\kappa_{1} \lambda x, \kappa_{2} \lambda x\right)\right|_{x=\tilde{x}} .
\end{aligned}
$$

Furthermore, putting together expressions (3.24), (3.52), (3.58) and (3.53), (3.56), respectively, we obtain for the complete decomposition of the light-cone vector operator the following results anticipated by eqs. (3.9) and (3.10), respectively:

$$
\begin{aligned}
& G_{(\alpha \bullet)}\left(\kappa_{1} \tilde{x}, \kappa_{2} \tilde{x}\right)=G_{(\alpha \bullet)}^{\mathrm{tw} 2}\left(\kappa_{1} \tilde{x}, \kappa_{2} \tilde{x}\right)+G_{(\alpha \bullet)}^{\mathrm{tw} 3}\left(\kappa_{1} \tilde{x}, \kappa_{2} \tilde{x}\right)+G_{(\alpha \bullet)}^{\mathrm{tw} 4}\left(\kappa_{1} \tilde{x}, \kappa_{2} \tilde{x}\right) \\
& G_{[\alpha \bullet]}\left(\kappa_{1} \tilde{x}, \kappa_{2} \tilde{x}\right)=G_{[\alpha \bullet]}^{\mathrm{tw} 3}\left(\kappa_{1} \tilde{x}, \kappa_{2} \tilde{x}\right)+G_{[\alpha \bullet]}^{\mathrm{tw} 4(\mathrm{ii})}\left(\kappa_{1} \tilde{x}, \kappa_{2} \tilde{x}\right)
\end{aligned}
$$




\subsection{Tensor operators of symmetry class (iii)}

\subsubsection{Construction of the nonlocal antisymmetric class-(iii) operators of definite twist}

Now we consider the twist -4 and twist -5 contributions originating from the (unique) Young tableau related to the symmetry class (iii) (with the normalizing factor $\beta_{n}$ ):

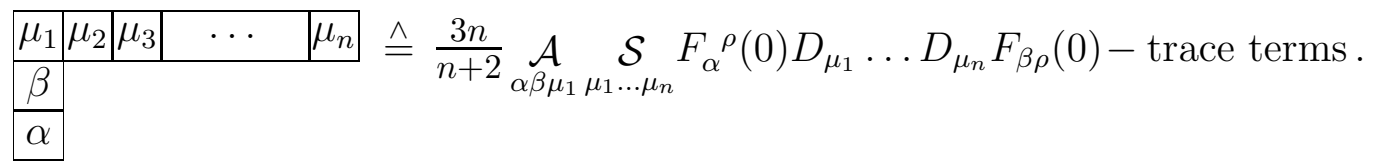

The corresponding traceless local operator having twist $\tau=4$ and being contained in the tensor space $\mathbf{T}\left(\frac{n}{2}, \frac{n}{2}\right)$ is given by:

$$
\begin{gathered}
G_{\alpha \beta \mu_{1} \ldots \mu_{n}}^{\mathrm{tw} 4(\mathrm{iii})}=\frac{n}{n+2}\left(F_{[\alpha \mid}^{\rho}(0) D_{\left(\mu_{1}\right.} \ldots D_{\left.\mu_{n}\right)} F_{\mid \beta] \rho}(0)-F_{[\alpha \mid}{ }^{\rho}(0) D_{\left(\beta \mu_{2}\right.} \ldots D_{\left.\mu_{n}\right)} F_{\left.\mid \mu_{1}\right] \rho}(0)\right. \\
\left.-F_{\left[\mu_{1} \mid\right.}{ }^{\rho}(0) D_{(\alpha} D_{\mu_{2}} \ldots D_{\left.\mu_{n}\right)} F_{\mid \beta] \rho}(0)\right)- \text { trace terms. }
\end{gathered}
$$

Now, contracting this expression with $x^{\mu_{1}} \ldots x^{\mu_{n}}$ we obtain:

$$
\begin{aligned}
G_{[\alpha \beta] n}^{\mathrm{tw} 4(\mathrm{iii})}(x) & =x^{\mu_{1}} \ldots x^{\mu_{n}} G_{[\alpha \beta] \mu_{1} \ldots \mu_{n}}^{\mathrm{tw} 4} \\
& =\frac{1}{n+2}\left((x \partial) \delta_{[\beta}^{\nu}-2 x^{\nu} \partial_{[\beta}\right) \delta_{\alpha]}^{\mu} \stackrel{\circ}{G}_{[\mu \nu] n}(x) .
\end{aligned}
$$

with the (antisymmetric) tensorial harmonic polynomials of order $n$ (see eq. (A.8)):

$$
\begin{aligned}
\stackrel{\circ}{G}_{[\alpha \beta] n}(x)= & \left\{\delta_{\alpha}^{\mu} \delta_{\beta}^{\nu}+\frac{1}{(n+1) n}\left(2 x_{[\alpha} \delta_{\beta]}^{[\mu} \partial^{\nu]}(x \partial)-x^{2} \partial_{[\alpha} \delta_{\beta]}^{[\mu} \partial^{\nu]}\right)\right. \\
& \left.-\frac{2}{(n+2)(n+1) n} x_{[\alpha} \partial_{\beta]} x^{[\mu} \partial^{\nu]}\right\} H_{n}^{(4)}\left(x^{2} \mid \square\right) G_{[\mu \nu] n}(x) .
\end{aligned}
$$

Resumming these local terms gives the nonlocal twist-4 operator as follows

$$
G_{[\alpha \beta]}^{\mathrm{tw} 4(\mathrm{iii})}(0, \kappa x)=\int_{0}^{1} \mathrm{~d} \lambda \lambda \delta_{[\alpha}^{\mu}\left((x \partial) \delta_{\beta]}^{\nu}-2 x^{\nu} \partial_{\beta]}\right) \stackrel{\circ}{G}_{[\mu \nu]}(0, \kappa \lambda x),
$$

where, using the integral representation of the additional factor $1 / n$ (the remaining factors of the denominator are taken together with $1 / n$ ! to get $1 /(n+2) !)$ and of the beta function, we introduced:

$$
\begin{aligned}
& \stackrel{\circ}{G}_{[\alpha \beta]}(0, \kappa x)=G_{[\alpha \beta]}(0, \kappa x)+\sum_{k=1}^{\infty} \int_{0}^{1} \frac{\mathrm{d} t}{t}\left(\frac{-x^{2}}{4}\right)^{k} \frac{\square^{k}}{k !(k-1) !}\left(\frac{1-t}{t}\right)^{k-1} G_{[\alpha \beta]}(0, \kappa t x) \\
& +\int_{0}^{1} \frac{\mathrm{d} \tau}{\tau}\left\{\left(2 x_{[\alpha} \delta_{\beta]}^{[\mu} \partial^{\nu]}(x \partial)-x^{2} \partial_{[\alpha} \delta_{\beta]}^{[\mu} \partial^{\nu]}\right) \sum_{k=0}^{\infty} \int_{0}^{1} \mathrm{~d} t\left(\frac{-x^{2}}{4}\right)^{k} \frac{\square^{k}}{k ! k !}\left(\frac{1-t}{t}\right)^{k}\right. \\
& \left.\quad-2 x_{[\alpha} \partial_{\beta]} x^{[\mu} \partial^{\nu]} \sum_{k=0}^{\infty} \int_{0}^{1} \mathrm{~d} t t\left(\frac{-x^{2}}{4}\right)^{k} \frac{\square^{k}}{(k+1) ! k !}\left(\frac{1-t}{t}\right)^{k+1}\right\} G_{[\mu \nu]}(0, \kappa \tau t x) .
\end{aligned}
$$


Then, by construction, eq. (3.61) defines a nonlocal operator of twist-4. As is easily seen by partial integration it fulfils the following relation

$$
G_{[\alpha \beta]}^{\mathrm{tw} 4(\mathrm{iii})}(0, \kappa x)=\stackrel{\circ}{G}_{[\alpha \beta]}(0, \kappa x)-G_{[\alpha \beta]}^{\mathrm{tw} 3}(0, \kappa x)
$$

and, because of eq. (3.33) and the properties of (3.62), it is a harmonic tensor operator:

$$
\square G_{[\alpha \beta]}^{\mathrm{tw} 4(\mathrm{iii})}(0, \kappa x)=0, \quad \partial^{\alpha} G_{[\alpha \beta]}^{\mathrm{tw} 4(\mathrm{iii})}(0, \kappa x)=0 .
$$

\subsubsection{Projection onto the light-cone}

Let us now project onto the light-cone. After the same calculations as has been carried out for the operator $M_{[\alpha \beta]}^{\mathrm{tw} 3}(0, \kappa \tilde{x})$ in Part I we obtain

$$
\begin{aligned}
G_{[\alpha \beta]}^{\mathrm{tw} 4(\mathrm{iii})}\left(\kappa_{1} \tilde{x}, \kappa_{2} \tilde{x}\right)= & \left.\int_{0}^{1} \mathrm{~d} \lambda \lambda \delta_{[\alpha}^{\mu}\left((x \partial) \delta_{\beta]}^{\nu}-2 x^{\nu} \partial_{\beta]}\right) G_{[\mu \nu]}\left(\kappa_{1} \lambda x, \kappa_{2} \lambda x\right)\right|_{x=\tilde{x}} \\
& -G_{[\alpha \beta]}^{\mathrm{tw} 5(\mathrm{iii})}\left(\kappa_{1} \tilde{x}, \kappa_{2} \tilde{x}\right),
\end{aligned}
$$

where the twist -5 part is determined by the trace terms, namely

$$
\begin{aligned}
G_{[\alpha \beta]}^{\mathrm{tw} 5(\mathrm{iii})}\left(\kappa_{1} \tilde{x}, \kappa_{2} \tilde{x}\right)=-\int_{0}^{1} \mathrm{~d} & \lambda \frac{1-\lambda^{2}}{\lambda}\left\{x_{[\alpha}\left(\delta_{\beta]}^{[\mu}(x \partial)-x^{[\mu} \partial_{\beta]}\right) \partial^{\nu]}\right. \\
& \left.-x_{[\alpha} \delta_{\beta]}^{[\mu} x^{\nu]} \square\right\}\left.G_{[\mu \nu]}\left(\kappa_{1} \lambda x, \kappa_{2} \lambda x\right)\right|_{x=\tilde{x}} .
\end{aligned}
$$

Obviously, there exist no vector (and scalar) operators of symmetry type (iii).

\subsubsection{Determination of the complete antisymmetric light-cone tensor operator}

This finishes the computation of twist contributions of the antisymmetric lightcone tensor operator. The complete antisymmetric twist-4 light-cone tensor operator obtains from eq. (3.65) and the twist -4 trace terms of the twist -3 operator, eq. (3.48):

$$
\begin{aligned}
& G_{[\alpha \beta]}^{\mathrm{tw} 4}\left(\kappa_{1} \tilde{x}, \kappa_{2} \tilde{x}\right)=G_{[\alpha \beta]}^{\mathrm{tw} 4(\mathrm{ii})}\left(\kappa_{1} \tilde{x}, \kappa_{2} \tilde{x}\right)+G_{[\alpha \beta]}^{\mathrm{tw} 4(\mathrm{iii})}\left(\kappa_{1} \tilde{x}, \kappa_{2} \tilde{x}\right) \\
& =\int_{0}^{1} \mathrm{~d} \lambda\left\{\lambda\left((x \partial) \delta_{[\beta}^{\nu}-2 x^{\nu} \partial_{[\beta}\right) \delta_{\alpha]}^{\mu}-\frac{1-\lambda^{2}}{\lambda}\left(x_{[\alpha} \partial_{\beta]} x^{[\mu} \partial^{\nu]}\right.\right. \\
& \left.\left.\quad+x_{[\alpha}\left(\delta_{\beta]}^{[\mu}(x \partial)-x^{[\mu} \partial_{\beta]}\right) \partial^{\nu]}-x_{[\alpha} \delta_{\beta]}^{[\mu} x^{\nu]} \square\right)\right\}\left.G_{[\mu \nu]}\left(\kappa_{1} \lambda x, \kappa_{2} \lambda x\right)\right|_{x=\tilde{x}} .
\end{aligned}
$$

Furthermore, the complete antisymmetric twist-5 light-cone tensor operator obtains from eqs. (3.49) and (3.66) as follows:

$$
\begin{aligned}
& G_{[\alpha \beta]}^{\mathrm{tw} 5}\left(\kappa_{1} \tilde{x}, \kappa_{2} \tilde{x}\right)=G_{[\alpha \beta]}^{\mathrm{tw} 5(\mathrm{ii})}\left(\kappa_{1} \tilde{x}, \kappa_{2} \tilde{x}\right)+G_{[\alpha \beta]}^{\mathrm{tw} 5(\mathrm{iii})}\left(\kappa_{1} \tilde{x}, \kappa_{2} \tilde{x}\right) \\
& \quad=\left.\int_{0}^{1} \mathrm{~d} \lambda \frac{1-\lambda}{\lambda}\left\{x_{[\alpha} \delta_{\beta]}^{[\mu} x^{\nu]} \square-2 x_{[\alpha}\left(\delta_{\beta]}^{[\mu}(x \partial)-x^{[\mu} \partial_{\beta]}\right) \partial^{\nu]}\right\} G_{[\mu \nu]}\left(\kappa_{1} \lambda x, \kappa_{2} \lambda x\right)\right|_{x=\tilde{x}} .
\end{aligned}
$$


Together with the twist-3 part, eq. (3.47), we finally obtain the complete decomposition of the antisymmetric light-cone tensor operator (compare eq. (3.8)):

$$
G_{[\alpha \beta]}\left(\kappa_{1} \tilde{x}, \kappa_{2} \tilde{x}\right)=G_{[\alpha \beta]}^{\mathrm{tw} 3}\left(\kappa_{1} \tilde{x}, \kappa_{2} \tilde{x}\right)+G_{[\alpha \beta]}^{\mathrm{tw} 4}\left(\kappa_{1} \tilde{x}, \kappa_{2} \tilde{x}\right)+G_{[\alpha \beta]}^{\mathrm{tw} 5}\left(\kappa_{1} \tilde{x}, \kappa_{2} \tilde{x}\right) .
$$

\subsection{Tensor operators of symmetry class (iv)}

\subsubsection{Construction of the nonlocal symmetric class-(iv) operator of definite twist}

Now let us investigate the symmetric tensor operators of the symmetry class (iv) which are given by the following Young tableau (with normalizing factor $\gamma_{n}$ ):

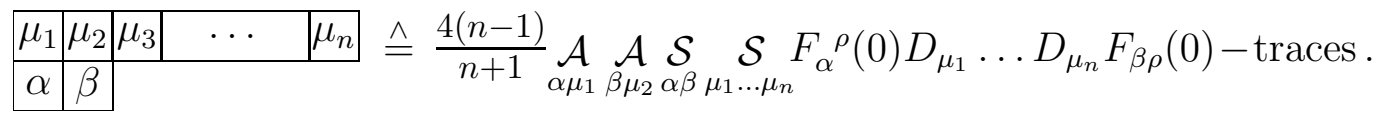

These local tensor operators have twist-4 and transform according to the representation $\mathbf{T}\left(\frac{n+2}{2}, \frac{n-2}{2}\right) \oplus \mathbf{T}\left(\frac{n-2}{2}, \frac{n+2}{2}\right)$. They are given by:

$$
\begin{gathered}
G_{\alpha \beta \mu_{1} \ldots \mu_{n}}^{\mathrm{tw} 4(\mathrm{iv})}=\frac{1}{(n+1) n}\left(G_{(\alpha \beta)\left(\mu_{1} \ldots \mu_{n}\right)}-G_{\left(\alpha \mu_{2}\right)\left(\mu_{1} \beta \mu_{3} \ldots \mu_{n}\right)}-G_{\left(\mu_{1} \beta\right)\left(\alpha \mu_{2} \ldots \mu_{n}\right)}\right. \\
\left.+G_{\left(\mu_{1} \mu_{2}\right)\left(\alpha \beta \mu_{3} \ldots \mu_{n}\right)}\right)- \text { trace terms }
\end{gathered}
$$

with $G_{(\alpha \beta)\left(\mu_{1} \ldots \mu_{n}\right)} \equiv F_{(\alpha \mid}^{\rho}(0) D_{\left(\mu_{1}\right.} \ldots D_{\left.\mu_{n}\right)} F_{\mid \beta) \rho}(0)$. Multiplying with $x^{\mu_{1}} \ldots x^{\mu_{n}}$ we obtain:

$$
\begin{aligned}
G_{(\alpha \beta) n}^{\mathrm{tw} 4(\mathrm{iv})}(x) & =x^{\mu_{1}} \ldots x^{\mu_{n}} G_{\alpha \beta \mu_{1} \ldots \mu_{n}}^{\mathrm{tw} 4(\mathrm{iv})} \\
& =\frac{1}{(n+1) n}\left(\delta_{\alpha}^{\mu} \delta_{\beta}^{\nu} x^{\rho}(x \partial) \partial_{\rho}-2 x^{\mu}(x \partial) \delta_{(\alpha}^{\nu} \partial_{\beta)}+x^{\mu} x^{\nu} \partial_{\alpha} \partial_{\beta}\right) \stackrel{\circ}{G}_{(\mu \nu) n}(x) .
\end{aligned}
$$

Here, $\stackrel{\circ}{G}_{(\mu \nu) n}(x)$ is the harmonic symmetric tensor polynomial of order $n$ (cf. Eq. (A.9), Appendix A):

$$
\begin{aligned}
\stackrel{\circ}{G}_{(\alpha \beta) n}(x)= & \left\{\delta_{\alpha}^{\mu} \delta_{\beta}^{\nu}+\frac{n}{(n+2)^{2}} g_{\alpha \beta} x^{(\nu} \partial^{\mu)}\right. \\
& -\frac{1}{(n+2)(n+1)}\left(2 n x_{(\alpha} \delta_{\beta)}^{(\nu} \partial^{\mu)}-x^{2} \delta_{(\alpha}^{(\nu} \partial_{\beta)} \partial^{\nu)}\right) \\
& -\frac{1}{(n+2)^{2}(n+1)}\left(2 n x_{(\alpha} \partial_{\beta)} x^{(\nu} \partial^{\mu)}-x^{2} \partial_{\alpha} \partial_{\beta} x^{(\nu} \partial^{\mu)}\right) \\
& +\frac{(n+3) n}{(n+2)^{2}(n+1)^{2}}\left(x_{\alpha} x_{\beta}-\frac{1}{2} x^{2} g_{\alpha \beta}\right) \partial^{\mu} \partial^{\nu} \\
& \left.-\frac{1}{(n+2)^{2}(n+1)^{2}}\left(2 x^{2} \partial_{(\alpha} x_{\beta)}+\frac{1}{4} x^{4} \partial_{\alpha} \partial_{\beta}\right) \partial^{\mu} \partial^{\nu}\right\} \breve{G}_{(\mu \nu) n}(x) .
\end{aligned}
$$

Resumming all local operators of symmetry class (iv) and using the integral representation $((n+1) n)^{-1}=\int_{0}^{1} \mathrm{~d} \lambda \lambda^{n}(1-\lambda) / \lambda$ gives

$$
\begin{gathered}
G_{(\alpha \beta)}^{\mathrm{tw} 4(\mathrm{iv})}(0, \kappa x)=\left(\delta_{\alpha}^{\mu} \delta_{\beta}^{\nu} x^{\rho}(x \partial) \partial_{\rho}-2 x^{\mu}(x \partial) \delta_{(\beta}^{\nu} \partial_{\alpha)}+x^{\mu} x^{\nu} \partial_{\alpha} \partial_{\beta}\right) \\
\times \int_{0}^{1} \mathrm{~d} \lambda \frac{1-\lambda}{\lambda} \stackrel{\circ}{G}_{(\mu \nu)}(0, \kappa \lambda x),
\end{gathered}
$$


where $\stackrel{\circ}{G}_{(\mu \nu)}(0, \kappa x)$ is given by

$$
\begin{gathered}
\stackrel{\circ}{G}_{(\alpha \beta)}(0, \kappa x)=\breve{G}_{(\alpha \beta)}(0, \kappa x)+\sum_{k=1}^{\infty} \int_{0}^{1} \frac{\mathrm{d} t}{t}\left(\frac{-x^{2}}{4}\right)^{k} \frac{\square^{k}}{k !(k-1) !}\left(\frac{1-t}{t}\right)^{k-1} \breve{G}_{(\alpha \beta)}(0, \kappa t x) \\
-\left[2 x_{(\alpha} \delta_{\beta)}^{(\mu} \partial^{\nu)}(x \partial)-x^{2} \partial_{(\alpha} \delta_{\beta)}^{(\mu} \partial^{\nu)}\right] \\
\quad \times \sum_{k=0}^{\infty} \int_{0}^{1} \mathrm{~d} t t\left(\frac{-x^{2}}{4}\right)^{k} \frac{\square^{k}}{k !(k+1) !}\left(\frac{1-t}{t}\right)^{k+1} \breve{G}_{(\mu \nu)}(0, \kappa t x) \\
-\left\{\left[2 x_{(\alpha} \partial_{\beta)} x^{(\mu} \partial^{\nu)}(x \partial)-g_{\alpha \beta} x^{(\mu} \partial^{\nu)}(x \partial)(x \partial+1)-x^{2} \partial_{\alpha} \partial_{\beta} x^{(\mu} \partial^{\nu)}\right] \int_{0}^{1} \mathrm{~d} \lambda \lambda\right. \\
\left.-\left[\left(x_{\alpha} x_{\beta}-\frac{x^{2}}{2} g_{\alpha \beta}\right) \partial^{\mu} \partial^{\nu}(x \partial)(x \partial+3)-\left(2 x^{2} \partial_{(\alpha} x_{\beta)}+\frac{x^{4}}{4} \partial_{\alpha} \partial_{\beta}\right) \partial^{\mu} \partial^{\nu}\right] \int_{0}^{1} \mathrm{~d} \lambda(1-\lambda)\right\} \\
\times \sum_{k=0}^{\infty} \int_{0}^{1} \mathrm{~d} t t\left(\frac{-x^{2}}{4}\right)^{k} \frac{\square}{(k+1) ! k !}\left(\frac{1-t}{t}\right)^{k+1} \breve{G}_{(\mu \nu)}(0, \kappa \lambda t x) .
\end{gathered}
$$

Of course, eq. (3.70) we may be rewritten as follows

$G_{(\alpha \beta)}^{\mathrm{tw} 4(\mathrm{iv})}(0, \kappa x)=\stackrel{\circ}{G}_{(\alpha \beta)}(0, \kappa x)-2 \int_{0}^{1} \mathrm{~d} \lambda \frac{1}{\lambda} \partial_{(\alpha} \stackrel{\circ}{G}_{\beta)}^{+}(0, \kappa \lambda x)+\int_{0}^{1} \mathrm{~d} \lambda \frac{1-\lambda}{\lambda} \partial_{\alpha} \partial_{\beta} \stackrel{\circ}{G}(0, \kappa \lambda x)$,

which will be used in the further considerations. Contrary to any of the formerly obtained symmetric twist-4 tensor operators, this operator is the 'true tensor part', i.e., not being proportional to $g_{\alpha \beta}$ or $x_{\alpha}$ and $x_{\beta}$. Obviously, this twist -4 operator satisfies the conditions of tracelessness

$$
\square G_{(\alpha \beta)}^{\mathrm{tw} 4(\mathrm{iv})}(0, \kappa x)=0, \quad \partial^{\alpha} G_{(\alpha \beta)}^{\mathrm{tw} 4(\mathrm{iv})}(0, \kappa x)=0, \quad g^{\alpha \beta} G_{(\alpha \beta)}^{\mathrm{tw} 4(\mathrm{iv})}(0, \kappa x) .
$$

\subsubsection{Projection onto the light-cone}

The symmetric twist-4 tensor operator of symmetry type (iv) on the light-cone is obtained as follows

$$
\begin{aligned}
G_{(\alpha \beta)}^{\mathrm{tw} 4(\mathrm{iv})}\left(\kappa_{1} \tilde{x}, \kappa_{2} \tilde{x}\right)= & \left(\delta_{\alpha}^{\mu} \delta_{\beta}^{\nu} x^{\rho}(x \partial) \partial_{\rho}-2 x^{\mu}(x \partial) \delta_{(\beta}^{\nu} \partial_{\alpha)}+x^{\mu} x^{\nu} \partial_{\alpha} \partial_{\beta}\right) \\
& \times\left.\int_{0}^{1} \mathrm{~d} \lambda \frac{1-\lambda}{\lambda} G_{(\mu \nu)}\left(\kappa_{1} \lambda x, \kappa_{2} \lambda x\right)\right|_{x=\tilde{x}}-G_{(\alpha \beta)}^{>(\mathrm{iv})}\left(\kappa_{1} \tilde{x}, \kappa_{2} \tilde{x}\right),
\end{aligned}
$$

where the trace terms of higher twist are given by

$$
\begin{aligned}
& G_{(\alpha \beta)}^{>(\mathrm{iv})}\left(\kappa_{1} \tilde{x}, \kappa_{2} \tilde{x}\right)=\int_{0}^{1} \mathrm{~d} \lambda\left\{\left(2 \lambda x_{(\alpha} \delta_{\beta)}^{\nu} \partial^{\mu}-(1-\lambda) x_{\alpha} x_{\beta} \partial^{\mu} \partial^{\nu}\right) G_{(\mu \nu)}\left(\kappa_{1} \lambda x, \kappa_{2} \lambda x\right)\right. \\
& \quad-\left(\frac{1}{\lambda} g_{\alpha \beta} \partial^{\mu}+\frac{1-\lambda^{2}}{\lambda} x_{(\alpha} \delta_{\beta)}^{\mu} \square-\frac{(1-\lambda)^{2}}{2 \lambda} x_{\alpha} x_{\beta} \square \partial^{\mu}\right) G_{\mu}^{+}\left(\kappa_{1} \lambda x, \kappa_{2} \lambda x\right) \\
& \quad+\left(\frac{1-\lambda}{2 \lambda} g_{\alpha \beta} \square+\frac{(1-\lambda)^{2}}{2 \lambda} x_{(\alpha} \partial_{\beta)} \square-\frac{1}{2}\left(\frac{1-\lambda^{2}}{2 \lambda}+\ln \lambda\right) x_{\alpha} x_{\beta} \square^{2}\right) G\left(\kappa_{1} \lambda x, \kappa_{2} \lambda x\right) \\
& \left.\quad+\left(\frac{1}{2 \lambda} g_{\alpha \beta}(x \partial)-\lambda x_{(\alpha} \partial_{\beta)}-\frac{\lambda \ln \lambda}{2} x_{\alpha} x_{\beta} \square\right) G_{\rho}^{\rho}\left(\kappa_{1} \lambda x, \kappa_{2} \lambda x\right)\right\}\left.\right|_{x=\tilde{x}} .
\end{aligned}
$$


The explicit twist decomposition of this operator by means of the Young tableaux (i) and (ii) gives

$$
\begin{aligned}
G_{(\alpha \beta)}^{>(\mathrm{iv})}\left(\kappa_{1} \tilde{x}, \kappa_{2} \tilde{x}\right) & =G_{(\alpha \beta)}^{\mathrm{tw} 4(\mathrm{iv}) \mathrm{a}}\left(\kappa_{1} \tilde{x}, \kappa_{2} \tilde{x}\right)+G_{(\alpha \beta)}^{\mathrm{tw} 4(\mathrm{iv}) \mathrm{b}}\left(\kappa_{1} \tilde{x}, \kappa_{2} \tilde{x}\right)+G_{(\alpha \beta)}^{\mathrm{tw} 4(\mathrm{iv}) \mathrm{c}}\left(\kappa_{1} \tilde{x}, \kappa_{2} \tilde{x}\right) \\
& +G_{(\alpha \beta)}^{\mathrm{tw} 4(\mathrm{iv}) \mathrm{d} 1}\left(\kappa_{1} \tilde{x}, \kappa_{2} \tilde{x}\right)+G_{(\alpha \beta)}^{\mathrm{tw} 4(\mathrm{iv}) \mathrm{d} 2}\left(\kappa_{1} \tilde{x}, \kappa_{2} \tilde{x}\right)+G_{(\alpha \beta)}^{\mathrm{tw} 4(\mathrm{iv}) \mathrm{e} 1}\left(\kappa_{1} \tilde{x}, \kappa_{2} \tilde{x}\right) \\
& +G_{(\alpha \beta)}^{\mathrm{tw} 4(\mathrm{iv}) \mathrm{e} 2}\left(\kappa_{1} \tilde{x}, \kappa_{2} \tilde{x}\right)+G_{(\alpha \beta)}^{\mathrm{tw} 4(\mathrm{iv}) \mathrm{f}}\left(\kappa_{1} \tilde{x}, \kappa_{2} \tilde{x}\right)+G_{(\alpha \beta)}^{\mathrm{tw} 4(\mathrm{iv}) \mathrm{h}}\left(\kappa_{1} \tilde{x}, \kappa_{2} \tilde{x}\right) \\
& +G_{(\alpha \beta)}^{\mathrm{tw} 5(\mathrm{iv}) \mathrm{d}}\left(\kappa_{1} \tilde{x}, \kappa_{2} \tilde{x}\right)+G_{(\alpha \beta)}^{\mathrm{tw} 5(\mathrm{iv}) \mathrm{e}}\left(\kappa_{1} \tilde{x}, \kappa_{2} \tilde{x}\right) \\
& +G_{(\alpha \beta)}^{\mathrm{tw} 6(\mathrm{iv}) \mathrm{a}}\left(\kappa_{1} \tilde{x}, \kappa_{2} \tilde{x}\right)+G_{(\alpha \beta)}^{\mathrm{tw} 6(\mathrm{iv}) \mathrm{b}}\left(\kappa_{1} \tilde{x}, \kappa_{2} \tilde{x}\right)+G_{(\alpha \beta)}^{\mathrm{tw} 6(\mathrm{iv}) \mathrm{c}}\left(\kappa_{1} \tilde{x}, \kappa_{2} \tilde{x}\right) \\
& +G_{(\alpha \beta)}^{\mathrm{tw} 6(\mathrm{iv}) \mathrm{d} 1}\left(\kappa_{1} \tilde{x}, \kappa_{2} \tilde{x}\right)+G_{(\alpha \beta)}^{\mathrm{tw} 6(\mathrm{iv}) \mathrm{d} 2}\left(\kappa_{1} \tilde{x}, \kappa_{2} \tilde{x}\right)+G_{(\alpha \beta)}^{\mathrm{tw} 6(\mathrm{iv}) \mathrm{d} 3}\left(\kappa_{1} \tilde{x}, \kappa_{2} \tilde{x}\right) \\
& +G_{(\alpha \beta)}^{\mathrm{tw} 6(\mathrm{iv}) \mathrm{d} 4}\left(\kappa_{1} \tilde{x}, \kappa_{2} \tilde{x}\right)+G_{(\alpha \beta)}^{\mathrm{tw} 6(\mathrm{iv}) \mathrm{e} 1}\left(\kappa_{1} \tilde{x}, \kappa_{2} \tilde{x}\right)+G_{(\alpha \beta)}^{\mathrm{tw} 6(\mathrm{iv}) \mathrm{e} 2}\left(\kappa_{1} \tilde{x}, \kappa_{2} \tilde{x}\right) \\
& +G_{(\alpha \beta)}^{\mathrm{tw} 6(\mathrm{iv}) \mathrm{e} 3}\left(\kappa_{1} \tilde{x}, \kappa_{2} \tilde{x}\right)+G_{(\alpha \beta)}^{\mathrm{tw} 6(\mathrm{iv}) \mathrm{e} 4}\left(\kappa_{1} \tilde{x}, \kappa_{2} \tilde{x}\right)+G_{(\alpha \beta)}^{\mathrm{tw} 6(\mathrm{iv}) \mathrm{f}}\left(\kappa_{1} \tilde{x}, \kappa_{2} \tilde{x}\right) \\
& +G_{(\alpha \beta)}^{\mathrm{tw} 6(\mathrm{iv}) \mathrm{h}}\left(\kappa_{1} \tilde{x}, \kappa_{2} \tilde{x}\right)+G_{(\alpha \beta)}^{\mathrm{tw} 6(\mathrm{iv}) \mathrm{k}}\left(\kappa_{1} \tilde{x}, \kappa_{2} \tilde{x}\right),
\end{aligned}
$$

with the following operators of well-defined twist

$$
\begin{aligned}
G_{(\alpha \beta)}^{\mathrm{tw} 4(\mathrm{iv}) \mathrm{a}}\left(\kappa_{1} \tilde{x}, \kappa_{2} \tilde{x}\right)= & \left.g_{\alpha \beta} \square \int_{0}^{1} \mathrm{~d} \lambda \frac{1-\lambda}{2 \lambda} G\left(\kappa_{1} \lambda x, \kappa_{2} \lambda x\right)\right|_{x=\tilde{x}}, \\
G_{(\alpha \beta)}^{\mathrm{tw} 4(\mathrm{iv}) \mathrm{b}}\left(\kappa_{1} \tilde{x}, \kappa_{2} \tilde{x}\right)= & \left.x_{(\alpha} \partial_{\beta)} \square \int_{0}^{1} \mathrm{~d} \lambda \frac{(1-\lambda)^{2}}{2 \lambda} G\left(\kappa_{1} \lambda x, \kappa_{2} \lambda x\right)\right|_{x=\tilde{x}} \\
& -G_{(\alpha \beta)}^{\mathrm{tw} 6(\mathrm{iv}) \mathrm{b}}\left(\kappa_{1} \tilde{x}, \kappa_{2} \tilde{x}\right), \\
G_{(\alpha \beta)}^{\mathrm{tw} 4(\mathrm{iv}) \mathrm{d} 11}\left(\kappa_{1} \tilde{x}, \kappa_{2} \tilde{x}\right)= & \left.x_{(\alpha} \partial_{\beta)} \square \int_{0}^{1} \mathrm{~d} \lambda\left(\frac{1-\lambda^{2}}{2 \lambda}+\frac{\ln \lambda}{\lambda}\right) G\left(\kappa_{1} \lambda x, \kappa_{2} \lambda x\right)\right|_{x=\tilde{x}} \\
& -G_{(\alpha \beta)}^{\mathrm{tw} 6(\mathrm{iv}) \mathrm{d} 1}\left(\kappa_{1} \tilde{x}, \kappa_{2} \tilde{x}\right),
\end{aligned}
$$

and

$$
\begin{aligned}
& G_{(\alpha \beta)}^{\mathrm{tw} 6(\mathrm{iv}) \mathrm{a}}\left(\kappa_{1} \tilde{x}, \kappa_{2} \tilde{x}\right)=-\left.\frac{1}{2} x_{\alpha} x_{\beta} \square^{2} \int_{0}^{1} \mathrm{~d} \lambda\left(\frac{1-\lambda^{2}}{2 \lambda}+\ln \lambda\right) G\left(\kappa_{1} \lambda x, \kappa_{2} \lambda x\right)\right|_{x=\tilde{x}}, \\
& G_{(\alpha \beta)}^{\mathrm{tw} 6(\mathrm{iv}) \mathrm{b}}\left(\kappa_{1} \tilde{x}, \kappa_{2} \tilde{x}\right)=-\left.\frac{1}{2} x_{\alpha} x_{\beta} \square^{2} \int_{0}^{1} \mathrm{~d} \lambda\left(\frac{1-\lambda}{\lambda}-\frac{1-\lambda^{2}}{4 \lambda}+\frac{\ln \lambda}{2 \lambda}\right) G\left(\kappa_{1} \lambda x, \kappa_{2} \lambda x\right)\right|_{x=\tilde{x}}, \\
& G_{(\alpha \beta)}^{\mathrm{tw} 6(\mathrm{iv}) \mathrm{d} 1}\left(\kappa_{1} \tilde{x}, \kappa_{2} \tilde{x}\right)=-\left.\frac{1}{4} x_{\alpha} x_{\beta} \square^{2} \int_{0}^{1} \mathrm{~d} \lambda\left(\frac{1-\lambda^{2}}{2 \lambda}+\frac{\ln \lambda}{\lambda}+\frac{\ln ^{2} \lambda}{\lambda}\right) G\left(\kappa_{1} \lambda x, \kappa_{2} \lambda x\right)\right|_{x=\tilde{x}}, \\
& G_{(\alpha \beta)}^{\mathrm{tw} 6(\mathrm{iv}) \mathrm{d} 3}\left(\kappa_{1} \tilde{x}, \kappa_{2} \tilde{x}\right)=\left.\frac{1}{2} x_{\alpha} x_{\beta} \square^{2} \int_{0}^{1} \mathrm{~d} \lambda\left(\frac{1-\lambda^{2}}{2 \lambda}+\frac{\ln \lambda}{\lambda}+\frac{\ln ^{2} \lambda}{\lambda}\right) G\left(\kappa_{1} \lambda x, \kappa_{2} \lambda x\right)\right|_{x=\tilde{x}},
\end{aligned}
$$


being related to the scalar operator, as well as

$$
\begin{aligned}
G_{(\alpha \beta)}^{\mathrm{tw} 4(\mathrm{iv}) \mathrm{c}}\left(\kappa_{1} \tilde{x}, \kappa_{2} \tilde{x}\right)= & -\left.g_{\alpha \beta} \partial^{\mu} \int_{0}^{1} \mathrm{~d} \lambda \frac{1}{\lambda} G_{\mu}^{+}\left(\kappa_{1} \lambda x, \kappa_{2} \lambda x\right)\right|_{x=\tilde{x}}, \\
G_{(\alpha \beta)}^{\mathrm{tw} 4(\mathrm{iv}) \mathrm{d} 2}\left(\kappa_{1} \tilde{x}, \kappa_{2} \tilde{x}\right)= & -\left.2 x_{(\alpha} \partial_{\beta} \partial^{\mu} \int_{0}^{1} \mathrm{~d} \lambda\left(\frac{1-\lambda^{2}}{2 \lambda}+\frac{\ln \lambda}{\lambda}\right) G_{\mu}^{+}\left(\kappa_{1} \lambda x, \kappa_{2} \lambda x\right)\right|_{x=\tilde{x}} \\
& -G_{(\alpha \beta)}^{\mathrm{tw} 6(\mathrm{iv}) \mathrm{d} 2}\left(\kappa_{1} \tilde{x}, \kappa_{2} \tilde{x}\right), \\
G_{(\alpha \beta)}^{\mathrm{tw} 4(\mathrm{iv}) \mathrm{e} 1}\left(\kappa_{1} \tilde{x}, \kappa_{2} \tilde{x}\right)= & \left.x_{(\alpha} \partial_{\beta)} \partial^{\mu} \int_{0}^{1} \mathrm{~d} \lambda \frac{1-\lambda^{2}}{\lambda} G_{\mu}^{+}\left(\kappa_{1} \lambda x, \kappa_{2} \lambda x\right)\right|_{x=\tilde{x}} \\
& -G_{(\alpha \beta)}^{\mathrm{tw} 6(\mathrm{iv}) \mathrm{e} 1}\left(\kappa_{1} \tilde{x}, \kappa_{2} \tilde{x}\right),
\end{aligned}
$$

and

$$
\begin{aligned}
G_{(\alpha \beta)}^{\mathrm{tw} 5(\mathrm{iv}) \mathrm{d}}\left(\kappa_{1} \tilde{x}, \kappa_{2} \tilde{x}\right)= & x_{(\alpha}\left(\delta_{\beta)}^{\mu}(x \partial)-x^{\mu} \partial_{\beta)}\right) \square \\
& \times\left.\int_{0}^{1} \mathrm{~d} \lambda\left(\frac{1-\lambda^{2}}{2 \lambda}+\frac{\ln \lambda}{\lambda}\right) G_{\mu}^{+}\left(\kappa_{1} \lambda x, \kappa_{2} \lambda x\right)\right|_{x=\tilde{x}} \\
& -G_{(\alpha \beta)}^{\mathrm{tw} 6(\mathrm{iv}) \mathrm{d} 3}\left(\kappa_{1} \tilde{x}, \kappa_{2} \tilde{x}\right)-G_{(\alpha \beta)}^{\mathrm{tw} 6(\mathrm{iv}) \mathrm{d} 4}\left(\kappa_{1} \tilde{x}, \kappa_{2} \tilde{x}\right)
\end{aligned}
$$

and

$$
\begin{aligned}
& G_{(\alpha \beta)}^{\mathrm{tw} 6(\mathrm{iv}) \mathrm{c}}\left(\kappa_{1} \tilde{x}, \kappa_{2} \tilde{x}\right)=\left.\frac{1}{2} x_{\alpha} x_{\beta} \square \partial^{\mu} \int_{0}^{1} \mathrm{~d} \lambda \frac{(1-\lambda)^{2}}{\lambda} G_{\mu}^{+}\left(\kappa_{1} \lambda x, \kappa_{2} \lambda x\right)\right|_{x=\tilde{x}}, \\
& G_{(\alpha \beta)}^{\mathrm{tw} 6(\mathrm{iv}) \mathrm{d} 2}\left(\kappa_{1} \tilde{x}, \kappa_{2} \tilde{x}\right)=\left.\frac{1}{2} x_{\alpha} x_{\beta} \square \partial^{\mu} \int_{0}^{1} \mathrm{~d} \lambda\left(\frac{1-\lambda^{2}}{2 \lambda}+\frac{\ln \lambda}{\lambda}+\frac{\ln ^{2} \lambda}{\lambda}\right) G_{\mu}^{+}\left(\kappa_{1} \lambda x, \kappa_{2} \lambda x\right)\right|_{x=\tilde{x}}, \\
& G_{(\alpha \beta)}^{\mathrm{tw} 6(\mathrm{iv}) \mathrm{d} 4}\left(\kappa_{1} \tilde{x}, \kappa_{2} \tilde{x}\right)=\left.x_{\alpha} x_{\beta} \square \partial^{\mu} \int_{0}^{1} \mathrm{~d} \lambda\left(\frac{1-\lambda^{2}}{2 \lambda}+\frac{\ln \lambda}{\lambda}\right) G_{\mu}^{+}\left(\kappa_{1} \lambda x, \kappa_{2} \lambda x\right)\right|_{x=\tilde{x}}, \\
& G_{(\alpha \beta)}^{\mathrm{tw} 6(\mathrm{iv}) \mathrm{e} 1}\left(\kappa_{1} \tilde{x}, \kappa_{2} \tilde{x}\right)=-\left.\frac{1}{2} x_{\alpha} x_{\beta} \square \partial^{\mu} \int_{0}^{1} \mathrm{~d} \lambda\left(\frac{1-\lambda^{2}}{2 \lambda}+\frac{\ln \lambda}{\lambda}\right) G_{\mu}^{+}\left(\kappa_{1} \lambda x, \kappa_{2} \lambda x\right)\right|_{x=\tilde{x}}, \\
& G_{(\alpha \beta)}^{\mathrm{tw} 6(\mathrm{iv}) \mathrm{e} 3}\left(\kappa_{1} \tilde{x}, \kappa_{2} \tilde{x}\right)=\left.x_{\alpha} x_{\beta} \square \partial^{\mu} \int_{0}^{1} \mathrm{~d} \lambda\left(\frac{1-\lambda^{2}}{2 \lambda}+\frac{\ln \lambda}{\lambda}\right) G_{\mu}^{+}\left(\kappa_{1} \lambda x, \kappa_{2} \lambda x\right)\right|_{x=\tilde{x}},
\end{aligned}
$$

being related to the symmetric vector operator,

$$
\begin{aligned}
G_{(\alpha \beta)}^{\mathrm{tw} 5(\mathrm{iv}) \mathrm{e}}\left(\kappa_{1} \tilde{x}, \kappa_{2} \tilde{x}\right)= & \left.x_{(\alpha}\left(\delta_{\beta)}^{\nu}(x \partial)-x^{\nu} \partial_{\beta)}\right) \partial^{\mu} \int_{0}^{1} \mathrm{~d} \lambda \frac{1-\lambda^{2}}{\lambda} G_{(\mu \nu)}\left(\kappa_{1} \lambda x, \kappa_{2} \lambda x\right)\right|_{x=\tilde{x}}, \\
& -G_{(\alpha \beta)}^{\mathrm{tw} 6(\mathrm{iv}) \mathrm{e} 3}\left(\kappa_{1} \tilde{x}, \kappa_{2} \tilde{x}\right) G_{(\alpha \beta)}^{\mathrm{tw} 6(\mathrm{iv}) \mathrm{e} 4}\left(\kappa_{1} \tilde{x}, \kappa_{2} \tilde{x}\right) \\
G_{(\alpha \beta)}^{\mathrm{tw} 6(\mathrm{iv}) \mathrm{e} 4}\left(\kappa_{1} \tilde{x}, \kappa_{2} \tilde{x}\right)= & \left.x_{\alpha} x_{\beta} \partial^{\mu} \partial^{\nu} \int_{0}^{1} \mathrm{~d} \lambda \frac{1-\lambda^{2}}{\lambda} G_{(\mu \nu)}\left(\kappa_{1} \lambda x, \kappa_{2} \lambda x\right)\right|_{x=\tilde{x}}, \\
G_{(\alpha \beta)}^{\mathrm{tw} 6(\mathrm{iv}) \mathrm{f}}\left(\kappa_{1} \tilde{x}, \kappa_{2} \tilde{x}\right)= & -\left.x_{\alpha} x_{\beta} \partial^{\mu} \partial^{\nu} \int_{0}^{1} \mathrm{~d} \lambda(1-\lambda) G_{(\mu \nu)}\left(\kappa_{1} \lambda x, \kappa_{2} \lambda x\right)\right|_{x=\tilde{x}},
\end{aligned}
$$


being related to the symmetric tensor operator and, furthermore,

$$
\begin{aligned}
G_{(\alpha \beta)}^{\mathrm{tw} 4(\mathrm{iv}) \mathrm{f}}\left(\kappa_{1} \tilde{x}, \kappa_{2} \tilde{x}\right) & =\left.g_{\alpha \beta}(x \partial) \int_{0}^{1} \mathrm{~d} \lambda \frac{1}{2 \lambda} G_{\rho}^{\rho}\left(\kappa_{1} \lambda x, \kappa_{2} \lambda x\right)\right|_{x=\tilde{x}}, \\
G_{(\alpha \beta)}^{\mathrm{tw} 4(\mathrm{iv}) \mathrm{h}}\left(\kappa_{1} \tilde{x}, \kappa_{2} \tilde{x}\right) & =-\left.x_{(\alpha} \partial_{\beta)} \int_{0}^{1} \mathrm{~d} \lambda \lambda G_{\rho}^{\rho}{ }_{\rho}\left(\kappa_{1} \lambda x, \kappa_{2} \lambda x\right)\right|_{x=\tilde{x}}-G_{(\alpha \beta)}^{\mathrm{tw} 6(\mathrm{iv}) \mathrm{h}}\left(\kappa_{1} \tilde{x}, \kappa_{2} \tilde{x}\right), \\
G_{(\alpha \beta)}^{\mathrm{tw} 4(\mathrm{iv}) \mathrm{e} 2}\left(\kappa_{1} \tilde{x}, \kappa_{2} \tilde{x}\right) & =-\left.x_{(\alpha} \partial_{\beta)} \int_{0}^{1} \mathrm{~d} \lambda \frac{1-\lambda^{2}}{\lambda} G_{\rho}^{\rho}\left(\kappa_{1} \lambda x, \kappa_{2} \lambda x\right)\right|_{x=\tilde{x}}-G_{(\alpha \beta)}^{\mathrm{tw} 6(\mathrm{iv}) \mathrm{e} 2}\left(\kappa_{1} \tilde{x}, \kappa_{2} \tilde{x}\right),
\end{aligned}
$$

and

$$
\begin{aligned}
& G_{(\alpha \beta)}^{\mathrm{tw} 6(\mathrm{iv}) \mathrm{h}}\left(\kappa_{1} \tilde{x}, \kappa_{2} \tilde{x}\right)=-\left.\frac{1}{4} x_{\alpha} x_{\beta} \square \int_{0}^{1} \mathrm{~d} \lambda \frac{1-\lambda^{2}}{\lambda} G_{\rho}^{\rho}\left(\kappa_{1} \lambda x, \kappa_{2} \lambda x\right)\right|_{x=\tilde{x}}, \\
& G_{(\alpha \beta)}^{\mathrm{tw} 6(\mathrm{iv}) \mathrm{e} 2}\left(\kappa_{1} \tilde{x}, \kappa_{2} \tilde{x}\right)=\left.\frac{1}{2} x_{\alpha} x_{\beta} \square \int_{0}^{1} \mathrm{~d} \lambda\left(\frac{1-\lambda^{2}}{2 \lambda}+\frac{\ln \lambda}{\lambda}\right) G_{\rho}^{\rho}\left(\kappa_{1} \lambda x, \kappa_{2} \lambda x\right)\right|_{x=\tilde{x}}, \\
& G_{(\alpha \beta)}^{\mathrm{tw} 6(\mathrm{iv}) \mathrm{k}}\left(\kappa_{1} \tilde{x}, \kappa_{2} \tilde{x}\right)=-\left.\frac{1}{2} x_{\alpha} x_{\beta} \square \int_{0}^{1} \mathrm{~d} \lambda \lambda(\ln \lambda) G_{\rho}^{\rho}\left(\kappa_{1} \lambda x, \kappa_{2} \lambda x\right)\right|_{x=\tilde{x}},
\end{aligned}
$$

being related to the trace of the symmetric tensor operator.

Here, it should be remarked that, contrary to the former cases (i) - (iii), the trace terms (3.74) contain operators having the same twist, $\tau=4$, as the primary operator of symmetry class (iv). However, these operators being multiplied by either $g_{\alpha \beta}$ or $x_{\alpha}$ and $x_{\beta}$ are scalar or vector operators corresponding to symmetry type (i). Whereas the local operators resulting from the twist-4 tensor operator of symmetry class (iv) are contained in the tensor space $\mathbf{T}\left(\frac{n+2}{2}, \frac{n-2}{2}\right) \oplus \mathbf{T}\left(\frac{n-2}{2}, \frac{n+2}{2}\right)$, the local operators of the twist -4 scalar and vector operators are totally symmetric traceless tensors and, therefore, contained in the tensor space $\mathbf{T}\left(\frac{n}{2}, \frac{n}{2}\right)$.

\subsubsection{Determination of the complete symmetric light-cone tensor op- erators}

Now, having finished the decomposition of a general 2nd rank tensor operator, let us sum up the remaining symmetric tensor operators of twist greater than 3 , which appear in the trace terms of the symmetric twist operators with Young symmetry (i), (ii) as well as (iv), to complete twist-4, twist-5 and twist-6 operators.

The 'scalar part' of the twist-4 light-ray tensor operator is given by

$$
\begin{aligned}
& G_{(\alpha \beta)}^{\mathrm{tw} 4, \mathrm{~s}}\left(\kappa_{1} \tilde{x}, \kappa_{2} \tilde{x}\right)=-g_{\alpha \beta}\left\{\frac{1}{2} \square \int_{0}^{1} \mathrm{~d} \lambda \lambda(\ln \lambda) G\left(\kappa_{1} \lambda x, \kappa_{2} \lambda x\right)\right. \\
& \left.\quad-(x \partial) \int_{0}^{1} \mathrm{~d} \lambda \frac{1}{2 \lambda} G_{\rho}^{\rho}\left(\kappa_{1} \lambda x, \kappa_{2} \lambda x\right)+\partial^{\mu} \int_{0}^{1} \mathrm{~d} \lambda \lambda G_{\mu}^{+}\left(\kappa_{1} \lambda x, \kappa_{2} \lambda x\right)\right\}\left.\right|_{x=\tilde{x}}
\end{aligned}
$$


and the 'vector part' of the twist-4 light-ray tensor operator reads

$$
\begin{aligned}
& G_{(\alpha \beta)}^{\mathrm{tw} 4, \mathrm{v}}\left(\kappa_{1} \tilde{x}, \kappa_{2} \tilde{x}\right)=-\tilde{x}_{(\alpha} \times \\
& \quad\left\{\int_{0}^{1} \mathrm{~d} \lambda\left\{\left(\frac{1-\lambda^{2}}{2 \lambda}+\lambda \ln \lambda\right) \partial_{\beta)}+\frac{1}{4} x_{\beta}\left(\frac{1-\lambda^{2}}{\lambda}+\lambda \ln \lambda+\frac{\ln \lambda}{\lambda}\right) \square\right\} \square G\left(\kappa_{1} \lambda x, \kappa_{2} \lambda x\right)\right. \\
& \quad+\int_{0}^{1} \frac{\mathrm{d} \lambda}{\lambda}\left\{\partial_{\beta)}+\frac{1}{2} x_{\beta)}(\ln \lambda) \square\right\} G^{\rho}{ }_{\rho}\left(\kappa_{1} \lambda x, \kappa_{2} \lambda x\right) \\
& \left.\quad-\int_{0}^{1} \frac{\mathrm{d} \lambda}{\lambda}\left\{2\left(1-\lambda^{2}\right) \partial_{\beta}+\frac{1}{2} x_{\beta)}\left(1-\lambda^{2}+2 \ln \lambda\right) \square\right\} \partial^{\mu} G_{\mu}^{+}\left(\kappa_{1} \lambda x, \kappa_{2} \lambda x\right)\right\}\left.\right|_{x=\tilde{x}} .
\end{aligned}
$$

Furthermore, the contributions to the complete 'vector part' of the twist-5 lightray tensor operator are constructed by means of the Young tableau (ii), and their local operators are antisymmetric traceless tensors contained in the space $\mathbf{T}\left(\frac{n}{2}, \frac{n-2}{2}\right) \oplus \mathbf{T}\left(\frac{n-2}{2}, \frac{n}{2}\right)$. This complete twist -5 tensor operator is given by

$$
\begin{aligned}
& G_{(\alpha \beta)}^{\mathrm{tw} 5 \mathrm{v}}\left(\kappa_{1} \tilde{x}, \kappa_{2} \tilde{x}\right)=-\tilde{x}_{(\alpha} \times \\
& \begin{aligned}
&\left\{\int _ { 0 } ^ { 1 } \mathrm { d } \lambda \left\{\frac{(1-\lambda)^{2}}{2 \lambda}\left(\delta_{\beta)}^{\nu}(x \partial)-x^{\nu} \partial_{\beta)}-x_{\beta)} \partial^{\nu}\right) \square-\right.\right.\left.\left(\frac{1-\lambda}{\lambda}-\frac{1-\lambda^{2}}{4 \lambda}+\frac{1}{2 \lambda} \ln \lambda\right) x_{\beta)} \square^{2} x^{\nu}\right\} \\
& \times G_{\nu}^{+}\left(\kappa_{1} \lambda x, \kappa_{2} \lambda x\right) \\
&-\int_{0}^{1} \mathrm{~d} \lambda\left\{\frac{1-\lambda^{2}}{\lambda}\left(\delta_{\beta)}^{\nu}(x \partial)-x^{\nu} \partial_{\beta)}-x_{\beta)} \partial^{\nu}\right) \partial^{\mu}-\left(\frac{1-\lambda^{2}}{2 \lambda}+\frac{\ln \lambda}{\lambda}\right) x_{\beta)} \square \partial^{\mu} x^{\nu}\right\} \\
&\left.\times G_{(\mu \nu)}\left(\kappa_{1} \lambda x, \kappa_{2} \lambda x\right)\right\}\left.\right|_{x=\tilde{x}}
\end{aligned}
\end{aligned}
$$

The 'scalar part' of the twist- 6 light-ray tensor operator has Young symmetry (i). Thus, the local twist -6 operators are totally symmetric traceless tensors lying in the space $\mathbf{T}\left(\frac{n-2}{2}, \frac{n-2}{2}\right)$. This nonlocal twist -6 operator is given by

$$
\begin{aligned}
G_{(\alpha \beta)}^{\mathrm{tw} 6, \mathrm{~s}}\left(\kappa_{1} \tilde{x}, \kappa_{2} \tilde{x}\right)=-\tilde{x}_{\alpha} \tilde{x}_{\beta}\left\{\frac{1}{2} \square^{2} \int_{0}^{1} \mathrm{~d} \lambda\left(\frac{1-\lambda}{\lambda}+\frac{1+\lambda}{2 \lambda} \ln \lambda\right) G\left(\kappa_{1} \lambda x, \kappa_{2} \lambda x\right)\right. \\
-\square \int_{0}^{1} \mathrm{~d} \lambda \frac{1-\lambda^{2}}{2 \lambda} G_{\rho}^{\rho}\left(\kappa_{1} \lambda x, \kappa_{2} \lambda x\right) \\
+\square \int_{0}^{1} \mathrm{~d} \lambda \frac{(1-\lambda)^{2}}{2 \lambda} \partial^{\mu} G_{\mu}^{+}\left(\kappa_{1} \lambda x, \kappa_{2} \lambda x\right) \\
\left.-\partial^{\mu} \partial^{\nu} \int_{0}^{1} \mathrm{~d} \lambda \frac{1-\lambda}{\lambda} G_{(\mu \nu)}\left(\kappa_{1} \lambda x, \kappa_{2} \lambda x\right)\right\}\left.\right|_{x=\tilde{x}} .
\end{aligned}
$$

Now, we may pick up all the contributions to the symmetric (traceless) tensor operator. Together with the twist-2 part, eq. (3.19), the twist-3 part, eq. (3.50),

\footnotetext{
${ }^{8}$ Note that antisymmetry is not in $\alpha$ and $\beta$ but results from the differential operator!
} 
and the twist-4 part, eq. (3.73), we finally obtain the following complete decomposition of the symmetric light-cone tensor operator (compare eq. (3.7)):

$$
\begin{aligned}
G_{(\alpha \beta)}\left(\kappa_{1} \tilde{x}, \kappa_{2} \tilde{x}\right) & =G_{(\alpha \beta)}^{\mathrm{tw} 2(\mathrm{i})}\left(\kappa_{1} \tilde{x}, \kappa_{2} \tilde{x}\right)+G_{(\alpha \beta)}^{\mathrm{tw} 3(\mathrm{ii})}\left(\kappa_{1} \tilde{x}, \kappa_{2} \tilde{x}\right)+G_{(\alpha \beta)}^{\mathrm{tw} 4(\mathrm{iv})}\left(\kappa_{1} \tilde{x}, \kappa_{2} \tilde{x}\right) \\
& +G_{(\alpha \beta)}^{\mathrm{tw} 4, \mathrm{~s}}\left(\kappa_{1} \tilde{x}, \kappa_{2} \tilde{x}\right)+G_{(\alpha \beta)}^{\mathrm{tw} 4, \mathrm{v}}\left(\kappa_{1} \tilde{x}, \kappa_{2} \tilde{x}\right) \\
& +G_{(\alpha \beta)}^{\mathrm{tw} 5, \mathrm{v}}\left(\kappa_{1} \tilde{x}, \kappa_{2} \tilde{x}\right)+G_{(\alpha \beta)}^{\mathrm{tw} 6, \mathrm{~s}}\left(\kappa_{1} \tilde{x}, \kappa_{2} \tilde{x}\right)
\end{aligned}
$$

Let us remark that by construction the terms of the first line are traceless, whereas the traces of the third line vanish because of antisymmetry and $\tilde{x}^{2}=0$ on the light-cone, respectively. However, the traces of the second line do not vanish but restore the trace of the tensor operator,

$$
g^{\alpha \beta} G_{\alpha \beta}\left(\kappa_{1} \tilde{x}, \kappa_{2} \tilde{x}\right)=g^{\alpha \beta} G_{(\alpha \beta)}^{\mathrm{tw} 4, \mathrm{~s}}\left(\kappa_{1} \tilde{x}, \kappa_{2} \tilde{x}\right)+g^{\alpha \beta} G_{(\alpha \beta)}^{\mathrm{tw} 4 \mathrm{v}}\left(\left(\kappa_{1} \tilde{x}, \kappa_{2} \tilde{x}\right)\right) .
$$

This may be proven explicitly by taking the trace of eqs. (3.77) and (3.78), using $(x \partial) \cong \lambda \partial_{\lambda}$, performing partial integrations and observing the equality

$$
\left.\left(g^{\alpha \beta}-2 \partial^{\alpha} x^{\beta}+(1 / 2) \square x^{\alpha} x^{\beta}\right) G_{(\alpha \beta)}\left(\kappa_{1} \lambda x, \kappa_{2} \lambda x\right)\right|_{\lambda=0}=0 .
$$

Herewith, the twist decomposition of a generic bilocal 2nd rank light-ray tensor operator is completed. In the next Chapter we show how this general formalism may be applied also to tri- and multi-local light-ray operators.

\section{Extension to trilocal tensor operators}

The procedure reviewed in Chapt. 2 for bilocal operators may be extended to trilocal operators, too. These operators occur in higher twist contributions to light-cone dominated processes and, additionally, as counterparts in the renormalization of such higher twist operators which have been obtained in Chapt. 3. In the following we consider various trilocal operators of minimal twist- 3 and twist-4. The local versions of such higher twist operators have been considered already in the early days of QCD in systematic studies of deep inelastic scattering [19, 20] and on behalf of giving some parton interpretation for the distribution amplitudes [21, 22]. Various studies of local twist-4 operators determined their anomalous dimension matrices and the behaviour of their structure functions 23]. The relevance of local twist- 3 operators for the structure function $g_{2}$ of polarized deep inelastic scattering is also well-known [24, 25]. If written nonlocal all these operators are necessarily multilocal. However, nonlocal higher twist operators have been extensively used only in the study of the structure function $g_{2}[13,26]$ and of the photon and vector meson wave functions [27, 28].

Here, our main interest is not to give an exhaustive study of the various scattering processes but to present the twist decomposition of some characteristic multilocal operators. 


\subsection{General trilocal tensor operators: Quark-antiquark- gluon, four-quark and four-gluon operators}

This Subsection is devoted to the consideration of trilocal operators which share the same twist decomposition as the gluon operators $G_{\alpha \beta}$ (or their (anti)symmetric parts). We call them unconstrained because their truncation with $x^{\alpha} x^{\beta}$ does not vanish identically. For compact notations, we will introduce the following abbreviations:

$$
\Gamma^{i}=\left\{1, \gamma_{5}\right\}, \quad \Gamma_{\alpha}^{i}=\left\{\gamma_{\alpha}, \gamma_{5} \gamma_{\alpha}\right\}, \quad \Gamma_{\alpha \beta}^{i}=\left\{\sigma_{\alpha \beta}, \gamma_{5} \sigma_{\alpha \beta}\right\}, \quad F_{\mu \nu}^{i}=\left\{F_{\mu \nu}, \widetilde{F}_{\mu \nu}\right\}
$$

\subsubsection{Quark-antiquark-gluon operators}

First, we consider the following quark-antiquark-gluon operators,? 9

$$
V_{\alpha \beta}^{i j}\left(\kappa_{1} x, \tau x, \kappa_{2} x\right)=\bar{\psi}\left(\kappa_{1} x\right) U\left(\kappa_{1} x, \tau x\right) \Gamma_{\alpha}^{i \rho} F_{\beta \rho}^{j}(\tau x) U\left(\tau x, \kappa_{2} x\right) \psi\left(\kappa_{2} x\right),
$$

and

$$
W_{[\alpha \beta]}^{i j}\left(\kappa_{1} x, \tau x, \kappa_{2} x\right)=\bar{\psi}\left(\kappa_{1} x\right) U\left(\kappa_{1} x, \tau x\right) \Gamma^{i} F_{\alpha \beta}^{j}(\tau x) U\left(\tau x, \kappa_{2} x\right) \psi\left(\kappa_{2} x\right),
$$

whose minimal twist is $\tau_{\text {min }}=3$ and 4 , respectively; they have the same symmetry as the gluon tensor operators $G_{\alpha \beta}$ and $G_{[\alpha \beta]}$, respectively.

In order to be able to apply the general procedure of Chapt. 2 as well as the results of Chapt. 3 we have to verify the local structure of these trilocal operators. Let us study, for example, the first of the above quark-gluon operators, $V_{\alpha \beta}^{11}$, in some detail and Taylor expand its three local fields around $y=0$ :

$$
\begin{aligned}
& V_{\alpha \beta}^{11}\left(\kappa_{1} x, \tau x, \kappa_{2} x\right)= \\
& \quad=\bar{\psi}\left(\kappa_{1} x\right) U\left(\kappa_{1} x, 0\right) U(0, \tau x) \sigma_{\alpha}^{\rho} F_{\beta \rho}(\tau x) U(\tau x, 0) U\left(0, \kappa_{2} x\right) \psi\left(\kappa_{2} x\right) \\
& =\left.\sum_{n_{1}, m, n_{2}=0}^{\infty} \frac{\kappa_{1}^{n_{1}} \tau^{m} \kappa_{2}^{n_{2}}}{n_{1} ! m ! n_{2} !}\left[\bar{\psi}(y)\left(\overleftarrow{D}_{y} x\right)^{n_{1}}\right] \sigma_{\alpha}{ }^{\rho}\left[\left(x D_{z}\right)^{m} F_{\beta \rho}(z)\right]\left[\left(x \vec{D}_{y}\right)^{n_{2}} \psi(y)\right]\right|_{y=z=0} \\
& =\sum_{N=0}^{\infty} \frac{1}{N !} \bar{\psi}(0)\left(\sum_{n=0}^{N} \sum_{\ell=0}^{n}\left(\begin{array}{c}
N \\
n
\end{array}\right)\left(\begin{array}{c}
n \\
\ell
\end{array}\right)\left(\kappa_{1} \overleftarrow{D} x\right)^{n-\ell} \sigma_{\alpha}^{\rho}\left[(\tau x D)^{N-n} F_{\beta \rho}(0)\right]\left(\kappa_{2} x \vec{D}\right)^{\ell}\right) \psi(0)
\end{aligned}
$$

where the left and right derivatives are given by eqs. (2.7), and

$$
D_{\mu} F_{\alpha \beta}=\partial_{\mu} F_{\alpha \beta}+\mathrm{i} g\left[A_{\mu}, F_{\alpha \beta}\right] .
$$

This leads to the following expansion into local tensor operators

$$
V_{\alpha \beta}^{11}\left(\kappa_{1} x, \tau x, \kappa_{2} x\right)=\sum_{N=0}^{\infty} \frac{1}{N !} x^{\mu_{1}} \ldots x^{\mu_{N}} V_{\alpha \beta \mu_{1} \ldots \mu_{N}}^{11}\left(\kappa_{1}, \tau, \kappa_{2}\right),
$$

\footnotetext{
${ }^{9}$ For simplicity we restrict to the nonsinglet case and suppress flavour matrices.
} 
where

$$
V_{\alpha \beta \mu_{1} \ldots \mu_{N}}^{11}\left(\kappa_{1}, \tau, \kappa_{2}\right) \equiv \bar{\psi}(0) \sigma_{\alpha}^{\rho} \mathbf{D}_{[\beta \rho] \mu_{1} \ldots \mu_{N}}\left(\kappa_{1}, \tau, \kappa_{2}\right) \psi(0),
$$

and the field strength-dependent generalized covariant derivative of $N$ th order is given by

$$
\begin{aligned}
& \mathbf{D}_{[\beta \rho] \mu_{1} \ldots \mu_{N}}\left(\kappa_{1}, \tau, \kappa_{2}\right)= \\
& \quad=N ! \sum_{n=0}^{N} \sum_{\ell=0}^{n} \frac{\kappa_{1}^{n-\ell}}{(n-\ell) !} \overleftarrow{D}_{\mu_{1}} \ldots \overleftarrow{D}_{\mu_{n-\ell}} \frac{\tau^{N-n}}{(N-n) !}\left[D_{\mu_{n+1}} \ldots D_{\mu_{N}} F_{\beta \rho}(0)\right] \frac{\kappa_{2}^{\ell}}{\ell !} \vec{D}_{\mu_{n-\ell+1}} \ldots \vec{D}_{\mu_{n}} .
\end{aligned}
$$

Obviously, if the field strength were not present this operation would reduce to the product $\stackrel{\leftrightarrow}{D}_{\mu_{1}}\left(\kappa_{1}, \kappa_{2}\right) \ldots \stackrel{\leftrightarrow}{D}_{\mu_{N}}\left(\kappa_{1}, \kappa_{2}\right)$ of generalized derivatives (2.7) introduced in Chapt. 2. Furthermore, for $\tau=0$ we are left with a more simple expression which, however, cannot be reduced to the $N$ th power of some extended derivative because $F$ is equipped with some matrix structure. Anyway, the local tensor operators (4.5) of rank $N+2$ with canonical dimension $d=N+5$, which are given as a sum of $\frac{1}{2}(N+1)(N+2)$ terms, have to be decomposed according to their geometric twist. In principle, this has to be done term-by-term. But, because of the linearity of that procedure we are not required to do this for any term explicitly.

Due to the same general local structure of $V_{\alpha \beta}^{i j}$ and $W_{\alpha \beta}^{i j}$ - which is governed by Taylor expansions completely analogous to eq. (4.4) - their decomposition into terms of definite twist leads to the same expressions as we obtained in Chapt. 3 but with $G_{\alpha \beta}$ and $G_{[\alpha \beta]}$ exchanged by $V_{\alpha \beta}^{i j}$ and $W_{\alpha \beta}^{i j}$, respectively. The only difference consists in a shift of any twist by one unit, $\tau \rightarrow \tau+1$, in the various inputs of Table 1 and 2 below.

In contrast to the operators $V$ and $W$ the quark-antiquark-gluon operators

$$
\mathcal{O}_{\alpha \beta}^{i j}\left(\kappa_{1} x, \tau x, \kappa_{2} x\right)=x^{\rho} \bar{\psi}\left(\kappa_{1} x\right) U\left(\kappa_{1} x, \tau x\right) \Gamma_{\alpha}^{i} F_{\beta \rho}^{j}(\tau x) U\left(\tau x, \kappa_{2} x\right) \psi\left(\kappa_{2} x\right)
$$

and

$$
\mathcal{O}_{\alpha \beta}^{\prime i j}\left(\kappa_{1} x, \tau x, \kappa_{2} x\right)=x^{\rho} x^{\sigma} \bar{\psi}\left(\kappa_{1} x\right) U\left(\kappa_{1} x, \tau x\right) \Gamma_{\alpha \rho}^{i} F_{\beta \sigma}^{j}(\tau x) U\left(\tau x, \kappa_{2} x\right) \psi\left(\kappa_{2} x\right)
$$

are special, namely, despite of their arbitrary symmetry with respect to $\alpha$ and $\beta$ they vanish identically if multiplied by $x^{\beta}$ and $x^{\alpha}$ or $x^{\beta}$, respectively. Therefore, they show some peculiarities which will be treated in Subsection 4.2.

\subsubsection{Four-quark and four-gluon operators}

Let us now consider trilocal operators which are built up from four quark or four gluon fields. We denote them by ${ }^{I} \mathcal{Q}_{\alpha \beta}^{i j}$ with $I=0$ and $I=2$, respectively (here, $I$ counts the number of external $x$ 's of the operators):

$$
\begin{aligned}
& { }^{I=0} \mathcal{Q}_{\alpha \beta}^{i j}\left(\kappa_{1} x, \tau x, \kappa_{2} x\right)= \\
& =\left(\bar{\psi}\left(\kappa_{1} x\right) \Gamma_{\alpha}^{i} U\left(\kappa_{1} x, \tau x\right) \psi(\tau x)\right)\left(\bar{\psi}(\tau x) U\left(\tau x, \kappa_{2} x\right) \Gamma_{\beta}^{j} \psi\left(\kappa_{2} x\right)\right)
\end{aligned}
$$


as well as

$$
\begin{aligned}
& { }^{I=2} \mathcal{Q}_{\alpha \beta}^{i j}\left(\kappa_{1} x, \tau x, \kappa_{2} x\right)=x^{\rho} x^{\sigma I=2} \mathcal{Q}_{\alpha \rho \sigma \beta}^{i j}\left(\kappa_{1} x, \tau x, \kappa_{2} x\right) \\
& \quad=x^{\rho} x^{\sigma}\left(F_{\alpha \mu}\left(\kappa_{1} x\right) U\left(\kappa_{1} x, \tau x\right) F_{\rho}^{i \mu}(\tau x)\right)\left(F_{\sigma}^{j \nu}(\tau x) U\left(\tau x, \kappa_{2} x\right) F_{\beta \nu}\left(\kappa_{2} x\right)\right) .
\end{aligned}
$$

Both sets of operators have minimal twist $\tau_{\min }=4$. Their local versions have been already considered by various authors, see e.g. [29]. The explicit form of the local operators will be given below. Because there are no restrictions concerning the free indices $\alpha$ and $\beta$ the Young patterns (i) - (iv) are involved. The twist decomposition may be performed along the lines of Chap. 3 and, as in the foregoing cases, the outcome will be almost the same as for $G_{\alpha \beta}$. Again, the difference is that now the value of twist raises by two units, $\tau \rightarrow \tau+2$, relative to the gluon operators shown in Table 1 and 2 of the Conclusions. In addition, because of the change in the external $x$-factors (not being accompanied by $\lambda$ ) the measures of the $\lambda$-integrations have to be changed according to (see also Subsection 4.2)

$$
\mathrm{d} \lambda \longrightarrow \mathrm{d} \lambda \lambda^{I}
$$

Open, up to now, is the structure of the local operators. Let us study in detail the first of the four-quark operators, ${ }^{I=0} \mathcal{Q}_{\alpha \beta}^{11}$, whose Taylor expansion may be written in two equivalent ways:

$$
\begin{aligned}
{ }^{I} & =0 \mathcal{Q}_{\alpha \beta}^{11}\left(\kappa_{1} x, \tau x, \kappa_{2} x\right)= \\
& =\left(\bar{\psi}\left(\kappa_{1} x\right) \gamma_{\alpha} U\left(\kappa_{1} x, 0\right) U(0, \tau x) \psi(\tau x)\right)\left(\bar{\psi}(\tau x) U(\tau x, 0) U\left(0, \kappa_{2} x\right) \gamma_{\beta} \psi\left(\kappa_{2} x\right)\right) \\
& =\left.\sum_{n_{1}, m, n_{2}=0}^{\infty} \frac{\kappa_{1}^{n_{1}} \tau^{m} \kappa_{2}^{n_{2}}}{n_{1} ! m ! n_{2} !}\left[\bar{\psi}(y)\left(\stackrel{\leftarrow}{D}_{y} x\right)^{n_{1}}\right] \gamma_{\alpha}\left[\left(x D_{z}\right)^{m}(\psi(z) \bar{\psi}(z))\right] \gamma_{\beta}\left[\left(x \vec{D}_{y}\right)^{n_{2}} \psi(y)\right]\right|_{y=z=0} \\
& =\left.\left.\sum_{n_{1}=0}^{\infty} \frac{1}{n_{1} !}\left(\bar{\psi}(y)\left(x \stackrel{\leftrightarrow}{D}_{y}\left(\kappa_{1}, \tau\right)\right)^{n_{1}} \gamma_{\alpha} \psi(y)\right)\right|_{y=0} \sum_{n_{2}=0}^{\infty} \frac{1}{n_{2} !}\left(\bar{\psi}(z)\left(x \stackrel{\leftrightarrow}{D}_{z}\left(\tau, \kappa_{2}\right)\right)^{n_{2}} \gamma_{\beta} \psi(z)\right)\right|_{z=0}
\end{aligned}
$$

where the two possibilities also use different generalized covariant derivatives. The left, right and left-right derivatives are given by eqs. (2.7), and, treating $\psi(0) \bar{\psi}(0)$ as a matrix in the group algebra, another form of the derivative obtains:

$$
\begin{aligned}
D_{\mu}(\psi(0) \bar{\psi}(0)) & =\partial_{\mu}(\psi(0) \bar{\psi}(0))+\mathrm{i} g\left[A_{\mu},(\psi(0) \bar{\psi}(0))\right] \\
& =\left(\vec{D}_{\mu} \psi(0)\right) \bar{\psi}(0)+\psi(0)\left(\bar{\psi}(0) \overleftarrow{D}_{\mu}\right)
\end{aligned}
$$

This leads to the following expansion into local tensor operators

$$
{ }^{I=0} \mathcal{Q}_{\alpha \beta}^{i j}\left(\kappa_{1} x, \tau x, \kappa_{2} x\right)=\sum_{N=0}^{\infty} \frac{1}{N !} x^{\mu_{1}} \ldots x^{\mu_{N} I=0} \mathcal{Q}_{\alpha \beta \mu_{1} \ldots \mu_{N}}^{i j}\left(\kappa_{1}, \tau, \kappa_{2}\right),
$$

where

$$
{ }^{I=0} \mathcal{Q}_{\alpha \beta \mu_{1} \ldots \mu_{N}}^{i j}\left(\kappa_{1}, \tau, \kappa_{2}\right) \equiv \bar{\psi}(0) \gamma_{\alpha} \mathbf{D}_{\mu_{1} \ldots \mu_{N}}\left(\kappa_{1}, \tau, \kappa_{2}\right) \gamma_{\beta} \psi(0)
$$


and the quark field-dependent generalized covariant derivative of $N$ th order is given by

$$
\begin{aligned}
\mathbf{D}_{\mu_{1} \ldots \mu_{N}}\left(\kappa_{1}, \tau, \kappa_{2}\right)=\sum_{n=0}^{N}\left(\begin{array}{c}
N \\
n
\end{array}\right) \stackrel{\leftrightarrow}{D}_{\mu_{1}} \ldots \stackrel{\leftrightarrow}{D}_{\mu_{n}} \psi(0) \times \bar{\psi}(0) \stackrel{\leftrightarrow}{D}_{\mu_{n+1}} \ldots \stackrel{\leftrightarrow}{D}_{\mu_{N}} \\
=\sum_{n=0}^{N} N ! \sum_{\ell=0}^{n} \overleftarrow{D}_{\mu_{1}} \ldots \overleftarrow{D}_{\mu_{n-\ell}} \frac{\kappa_{1}^{n-\ell} \tau^{\ell}}{(n-\ell) ! \ell !} \vec{D}_{\mu_{n-\ell+1}} \ldots \vec{D}_{\mu_{n}} \psi(0) \\
\quad \times \sum_{k}^{N-n} \bar{\psi}(0) \overleftarrow{D}_{\mu_{n+1}} \ldots \overleftarrow{D}_{\mu_{n+k}} \frac{\tau^{k} \kappa_{2}^{N-n-k}}{k ![N-n-k) !} \vec{D}_{\mu_{n+k+1}} \ldots \vec{D}_{\mu_{N}}
\end{aligned}
$$

Analogous generalized covariant derivatives occur for the four-gluon operators. The only difference will be that instead of the quark fields corresponding gluon field strengths appear in (4.14), and that the derivatives are to be taken in the adjoint representation. Despite having a complicated structure this does not matter in the twist decomposition of the trilinear operators ${ }^{I} \mathcal{Q}_{\alpha \beta}^{i j}\left(\kappa_{1} x, \tau x, \kappa_{2} x\right)$.

Let us finish this Subsection with a short remark concerning so-called fourparticle operators which also have been considered in the literature for local operators. These operators have the general structure, cf. [21, 22, 30]

$$
(\bar{\psi} U \psi)(\bar{\psi} U \psi), \quad(\bar{\psi} U F U F U \psi) \quad \text { or } \quad(F U F U F U F),
$$

with any field being located at a different point $\kappa_{i} x, i=1, \ldots, 4$. The expansion into local tensors is obtained in the same way as above, leading to a general expression of the following form:

$$
{ }^{I} \mathcal{R}_{\alpha \beta}\left(\kappa_{i} x\right)=\sum_{N=0}^{\infty} \frac{1}{N !} x^{\mu_{1}} \ldots x^{\mu_{N}} x^{\rho} \ldots x^{\sigma{ }^{I}} \mathcal{R}_{\alpha \beta \rho \ldots \sigma \mu_{1} \ldots \mu_{N}}\left(\kappa_{i}\right),
$$

where the terms with equal value of $N$ are given as well-defined sums of local tensor operators. Any of these operators decompose in the same manner into irreducible representations of the Lorentz group. The latter result is immediately related to the fact that all indices $\mu_{1} \ldots \mu_{N}$ are to be symmetrized, i.e., lying in the first row of any relevant Young tableau! Different symmetry classifications solely depend on the distribution of the remaining indices $\alpha \beta \rho \ldots \sigma$ - the others being somehow truncated - to the various Young tableaux. As long as only up to two free indices $\alpha \beta$ are relevant the twist decomposition takes place according to the results of Chapt. 3, eventually modified by the power $\lambda^{I}$ which is related to the number of external $x$ 's in ${ }^{I} \mathcal{R}$. In addition, the twist of the various components may be shifted by some (equal) amount.

\subsection{Constrained trilocal operators: Shuryak-Vainshtein- and three--gluon operators}

Now we consider the special quark-antiquark-gluon operator $\mathcal{O}_{\alpha \beta}^{i j}$, eq. (4.7), and related three-gluon operators both having minimal twist- 3 ; they will be denoted 
by ${ }^{I} \mathcal{O}_{\alpha \beta}^{i j}\left(\kappa_{1} x, \tau x, \kappa_{2} x\right)$ with $I=0$ and $I=1$, respectively. The quark-antiquarkgluon operators are given by

$$
\begin{aligned}
{ }^{I=0} \mathcal{O}_{\alpha \beta}^{i j}\left(\kappa_{1} x, \tau x, \kappa_{2} x\right) & =x^{\rho I=0} \mathcal{O}_{\alpha \beta \rho}^{i j}\left(\kappa_{1} x, \tau x, \kappa_{2} x\right) \\
& =x^{\rho} \bar{\psi}\left(\kappa_{1} x\right) U\left(\kappa_{1} x, \tau x\right) \Gamma_{\alpha}^{i} F_{\beta \rho}^{j}(\tau x) U\left(\tau x, \kappa_{2} x\right) \psi\left(\kappa_{2} x\right),
\end{aligned}
$$

where again some possible flavour structure has been suppressed. These operators are related to the following generalizations of the so-called Shuryak-Vainshtein operators [25, 13],

$$
S_{\beta}^{ \pm}\left(\kappa_{1} x, \tau x, \kappa_{2} x\right)=x^{\alpha}\left({ }^{I=1} \mathcal{O}_{\alpha \beta}^{11}\left(\kappa_{1} x, \tau x, \kappa_{2} x\right) \pm \mathrm{i}^{I=1} \mathcal{O}_{\alpha \beta}^{22}\left(\kappa_{1} x, \tau x, \kappa_{2} x\right)\right) x^{\alpha},
$$

which, in the flavour singlet case, mix with the following three-gluon operators:

$$
\begin{aligned}
& { }^{I=1} \mathcal{O}_{\alpha \beta}^{i}\left(\kappa_{1} x, \tau x, \kappa_{2} x\right)=x^{\rho} x^{\sigma}{ }^{I=1} \mathcal{O}_{\alpha \beta \rho \sigma}^{i}\left(\kappa_{1} x, \tau x, \kappa_{2} x\right) \\
& =x^{\rho} x^{\sigma} F_{\alpha \nu}^{a}\left(\kappa_{1} x\right) U^{a b}\left(\kappa_{1} x, \tau x\right) F_{\beta \rho}^{i b c}(\tau x) U^{c d}\left(\tau x, \kappa_{2} x\right) F_{\sigma}^{d \nu}\left(\kappa_{2} x\right)
\end{aligned}
$$

where $F_{\alpha \beta}^{a b}(x) \equiv f^{a c b} F_{\alpha \beta}^{c}(x)$ and the phase factors are taken in the adjoint representation.

Because of their construction these special trilocal operators have the property

$$
x^{\beta}{ }^{I} \mathcal{O}_{\alpha \beta}^{i j}\left(\kappa_{1} x, \tau x, \kappa_{2} x\right) \equiv 0 \text {. }
$$

They contain twist- 3 up to twist- 6 contributions which, because of the antisymmetry of the gluon field strength, have to be determined by means of the Young tableaux (ii) - (iv). Because of (4.18) the scalar operators $x^{\alpha} x^{\beta}{ }^{I} \mathcal{O}_{\alpha \beta}^{i j}\left(\kappa_{1} x, \tau x, \kappa_{2} x\right)$ vanish identically.

The Taylor expansion of the operator ${ }^{I=0} \mathcal{O}_{\alpha \beta}^{11}\left(\kappa_{1} x, \tau x, \kappa_{2} x\right)$ reads

$$
{ }^{I=0} \mathcal{O}_{\alpha \beta}^{11}\left(\kappa_{1} x, \tau x, \kappa_{2} x\right)=\sum_{N=0}^{\infty} \frac{1}{N !} x^{\mu_{1}} \ldots x^{\mu_{N}} x^{\rho I=0} \mathcal{O}_{\alpha \beta \rho \mu_{1} \ldots \mu_{N}}^{11}\left(\kappa_{1}, \tau, \kappa_{2}\right),
$$

with

$$
{ }^{I=0} \mathcal{O}_{\alpha \beta \rho \mu_{1} \ldots \mu_{N}}^{11}\left(\kappa_{1}, \tau, \kappa_{2}\right) \equiv \bar{\psi}(0) \gamma_{\alpha} \mathbf{D}_{[\beta \rho] \mu_{1} \ldots \mu_{N}}\left(\kappa_{1}, \tau, \kappa_{2}\right) \psi(0)
$$

where the field strength-dependent derivative already has been given by (4.6). The Taylor expansion of the related three-gluon operator obtains as follows:

$$
{ }^{I=1} \mathcal{O}_{\alpha \beta}^{11}\left(\kappa_{1} x, \tau x, \kappa_{2} x\right)=\sum_{N=0}^{\infty} \frac{1}{N !} x^{\mu_{1}} \ldots x^{\mu_{N}} x^{\rho} x^{\sigma \quad I=1} \mathcal{O}_{\alpha \beta \rho \sigma \mu_{1} \ldots \mu_{N}}^{A}\left(\kappa_{1}, \tau, \kappa_{2}\right)
$$

where

$$
{ }^{I=1} \mathcal{O}_{\alpha \beta \rho \sigma \mu_{1} \ldots \mu_{N}}^{11}\left(\kappa_{1}, \tau, \kappa_{2}\right) \equiv F_{\alpha \nu}^{a}(0) \mathbf{D}_{[\beta \rho] \mu_{1} \ldots \mu_{N}}^{a b}\left(\kappa_{1}, \tau, \kappa_{2}\right) F_{\sigma}^{b \nu}(0)
$$


with the same field strength dependent generalized covariant derivative (4.6), but now in the adjoint representation. The generalization arbitrary values $i$ and $j$ is obvious.

As it became obvious by the above considerations all the nonlocal threeparticle operators ${ }^{I} \mathcal{O}_{\alpha \beta}^{i j}$ have the same general local structure and, therefore, decompose according to the same symmetry patterns. They only differ in the rank of the local tensor operators which is due to the external powers of $x$. Again, this must be taken into account by a change of the integration measure according tot

$$
\mathrm{d} \lambda \longrightarrow \mathrm{d} \lambda \lambda^{I+1} .
$$

From now on we omit the indices $i$ and $j$.

In order to determine the twist- 3 light-cone operators ${ }^{I} \mathcal{O}_{\alpha \beta}\left(\kappa_{1} \tilde{x}, \tau \tilde{x}, \kappa_{2} \tilde{x}\right)$ for $I=0$ and $I=1$, let us consider the following Young tableaux of symmetry pattern \begin{tabular}{|l|l|l|l|l|}
\hline$\rho$ & $\mu_{1}$ & $\cdots$ & $\mu_{N}$ & $\alpha$ \\
\hline$\beta$ & &
\end{tabular} and \begin{tabular}{|l|l|l|l|l|}
\hline & $\sigma$ & $\mu_{1}$ & $\cdots$ & $\mu_{N} \mid \alpha$ \\
\hline$\beta$ & & &
\end{tabular} , respectively:

$$
\begin{aligned}
{ }^{I} \mathcal{O}_{\alpha \beta}^{\mathrm{tw} 3}\left(\kappa_{1} x, \tau x, \kappa_{2} x\right) & =\frac{1}{2} \int_{0}^{1} \mathrm{~d} \lambda \lambda^{I}\left[\left(1+\lambda^{2}\right) \delta_{\beta}^{\mu} \partial_{\alpha}+\left(1-\lambda^{2}\right) \delta_{\alpha}^{\mu} \partial_{\beta}\right]{ }^{I} \mathcal{O}_{\mu}\left(\kappa_{1} \lambda x, \tau \lambda x, \kappa_{2} \lambda x\right) \\
& ={ }^{I} \mathcal{O}_{[\alpha \beta]}^{\mathrm{tw} 3}\left(\kappa_{1} x, \tau x, \kappa_{2} x\right)+{ }^{I} \mathcal{O}_{(\alpha \beta)}^{\mathrm{tw} 3}\left(\kappa_{1} x, \tau x, \kappa_{2} x\right) ;
\end{aligned}
$$

here, and in the following, we write $\mathcal{O}_{\mu} \equiv x^{\rho} \mathcal{O}_{\rho \mu}$. Let us remark that, first, because of property (4.18), only the above mentioned Young tableaux contribute and, second, the difference between the expressions (4.23) and (3.42) result also from that property, namely the truncation by $x^{\rho}$ in the second term of the integrand, $\left.-x^{\mu} \partial_{\alpha} \partial_{\beta}{ }^{I} \stackrel{\circ}{\mathcal{O}}_{\mu}\left(\kappa_{1} x, \tau x, \kappa_{2} x\right)=2 \delta_{(\alpha}^{\mu} \partial_{\beta}\right)^{I^{\circ}} \mathcal{O}_{\mu}\left(\kappa_{1} x, \tau x, \kappa_{2} x\right)$, after partial integrations leads to the above expression (4.23). In the case $I=0$ this operator is in agreement with the expression given by Balitsky and Braun (cf. eq. (5.14) of [13]). However, their operator lacks to be really of twist -3 since it is not traceless; therefore it contains also twist -4 , twist -5 as well as twist- 6 operators resulting from the trace terms.

Now, let us to project onto the light-cone. As in the case of the gluon tensor only the first terms, $k=0,1,2$, in the expansion of ${ }^{{ }^{\circ}}{ }_{\mu}\left(\kappa_{1} \lambda x, \tau \lambda x, \kappa_{2} \lambda x\right)$ are to be taken into account. For the antisymmetric part of the twist- 3 operator one gets

$$
{ }^{I} \mathcal{O}_{[\alpha \beta]}^{\text {tw } 3}\left(\kappa_{1} \tilde{x}, \tau \tilde{x}, \kappa_{2} \tilde{x}\right)=\int_{0}^{1} \mathrm{~d} \lambda \lambda^{2+I} \partial_{[\alpha}{ }^{I} \mathcal{O}_{\beta]}\left(\kappa_{1} \lambda \tilde{x}, \tau \lambda \tilde{x}, \kappa_{2} \lambda \tilde{x}\right)-{ }^{I} \mathcal{O}_{[\alpha \beta]}^{>(\mathrm{ii})}\left(\kappa_{1} \tilde{x}, \tau \tilde{x}, \kappa_{2} \tilde{x}\right)
$$

where

$$
\begin{aligned}
{ }^{I} \mathcal{O}_{[\alpha \beta]}^{>(\mathrm{ii})}\left(\kappa_{1} \tilde{x}, \tau \tilde{x}, \kappa_{2} \tilde{x}\right)= & \frac{1}{2} \int_{0}^{1} \mathrm{~d} \lambda \lambda^{1+I}(1-\lambda)\left(2 x_{[\alpha} \partial_{\beta]} \partial^{\mu}-x_{[\alpha} \delta_{\beta]}^{\mu} \square\right) \\
& \times\left.{ }^{I} \mathcal{O}_{\mu}\left(\kappa_{1} \lambda x, \tau \lambda x, \kappa_{2} \lambda x\right)\right|_{x=\tilde{x}} .
\end{aligned}
$$

${ }^{10}$ Observe, that because of notational simplicity in the explicit expressions below we shifted the variable $I \rightarrow I-1$. 
The decomposition of ${ }^{I} \mathcal{O}_{[\alpha \beta]}^{>(\mathrm{ii})}\left(\kappa_{1} \tilde{x}, \tau \tilde{x}, \kappa_{2} \tilde{x}\right)$ arises as

$$
{ }^{I} \mathcal{O}_{[\alpha \beta]}^{>(\mathrm{ii})}\left(\kappa_{1} \tilde{x}, \tau \tilde{x}, \kappa_{2} \tilde{x}\right)={ }^{I} \mathcal{O}_{[\alpha \beta]}^{\mathrm{tw} 4(\mathrm{ii})}\left(\kappa_{1} \tilde{x}, \tau \tilde{x}, \kappa_{2} \tilde{x}\right)+{ }^{I} \mathcal{O}_{[\alpha \beta]}^{\mathrm{tw} 5(\mathrm{ii})}\left(\kappa_{1} \tilde{x}, \tau \tilde{x}, \kappa_{2} \tilde{x}\right),
$$

with

$$
\begin{aligned}
&{ }^{I} \mathcal{O}_{[\alpha \beta]}^{\mathrm{tw} 4(\mathrm{ii})}\left(\kappa_{1} \tilde{x}, \tau \tilde{x}, \kappa_{2} \tilde{x}\right)=\left.\frac{1}{2} x_{[\alpha} \partial_{\beta]} \partial^{\mu} \int_{0}^{1} \mathrm{~d} \lambda \lambda^{I}\left(1-\lambda^{2}\right){ }^{I} \mathcal{O}_{\mu}\left(\kappa_{1} \lambda x, \tau \lambda x, \kappa_{2} \lambda x\right)\right|_{x=\tilde{x}}, \\
&{ }^{I} \mathcal{O}_{[\alpha \beta]}^{\mathrm{tw} 5(\mathrm{ii})}\left(\kappa_{1} \tilde{x}, \tau \tilde{x}, \kappa_{2} \tilde{x}\right)=-\frac{1}{4} x_{[\alpha}\left(\delta_{\beta]}^{\mu}(x \partial)-x^{\mu} \partial_{\beta]}\right) \square \\
& \times\left.\int_{0}^{1} \mathrm{~d} \lambda \lambda^{I}(1-\lambda)^{2} \mathcal{O}_{\mu}\left(\kappa_{1} \lambda x, \tau \lambda x, \kappa_{2} \lambda x\right)\right|_{x=\tilde{x}} .
\end{aligned}
$$

The symmetric part reads

$$
{ }^{I} \mathcal{O}_{(\alpha \beta)}^{\mathrm{tw} 3}\left(\kappa_{1} \tilde{x}, \tau \tilde{x}, \kappa_{2} \tilde{x}\right)=\int_{0}^{1} \mathrm{~d} \lambda \lambda^{I} \partial_{(\alpha}{ }^{I} \mathcal{O}_{\beta)}\left(\kappa_{1} \lambda \tilde{x}, \tau \lambda \tilde{x}, \kappa_{2} \lambda \tilde{x}\right)-{ }^{I} \mathcal{O}_{(\alpha \beta)}^{>(\mathrm{ii})}\left(\kappa_{1} \tilde{x}, \tau \tilde{x}, \kappa_{2} \tilde{x}\right)
$$

where

$$
\begin{gathered}
{ }^{I_{\mathcal{O}}}>(\mathrm{ii}) \\
\left(\kappa_{1} \tilde{x}, \tau \tilde{x}, \kappa_{2} \tilde{x}\right)=\int_{0}^{1} \mathrm{~d} \lambda \lambda^{I}\left\{\frac{1}{2}\left(1-\lambda^{2}\right) g_{\alpha \beta} \partial^{\mu}+\frac{1}{2}(1-\lambda) \delta_{(\alpha}^{\mu} x_{\beta} \square\right. \\
\left.\left.+\lambda(1-\lambda) x_{(\alpha} \partial_{\beta)} \partial^{\mu}-\frac{1}{4}(1-\lambda)^{2} x_{\alpha} x_{\beta} \partial^{\mu} \square\right\}\right\}\left.^{I} \mathcal{O}_{\mu}\left(\kappa_{1} \lambda x, \tau \lambda x, \kappa_{2} \lambda x\right)\right|_{x=\tilde{x}}
\end{gathered}
$$

Let us remark that the conditions of tracelessness for the light-cone operators are:

$$
\begin{aligned}
g^{\alpha \beta I} \mathcal{O}_{(\alpha \beta)}^{\mathrm{tw} 3}\left(\kappa_{1} \tilde{x}, \tau \tilde{x}, \kappa_{2} \tilde{x}\right)=0, \quad \mathrm{~d}^{\alpha I} \mathcal{O}_{(\alpha \beta)}^{\mathrm{tw} 3}\left(\kappa_{1} \tilde{x}, \tau \tilde{x}, \kappa_{2} \tilde{x}\right) & =0, \\
\mathrm{~d}^{\alpha} \mathcal{O}_{[\alpha \beta]}^{\mathrm{tw} 3}\left(\kappa_{1} \tilde{x}, \tau \tilde{x}, \kappa_{2} \tilde{x}\right) & =0 .
\end{aligned}
$$

Now we can calculate the twist- 3 vector operator from the twist-3 tensor operator. The twist-3 light-cone vector operator reads

$$
\begin{gathered}
{ }^{I} \mathcal{O}_{\alpha}^{\mathrm{tw} 3}\left(\kappa_{1} \tilde{x}, \tau \tilde{x}, \kappa_{2} \tilde{x}\right)=\left.\int_{0}^{1} \mathrm{~d} \lambda \lambda^{2+I}\left[\delta_{\alpha}^{\mu}(x \partial)-x^{\mu} \partial_{\alpha}-x_{\alpha} \partial^{\mu}\right]{ }^{I} \mathcal{O}_{\mu}\left(\kappa_{1} \lambda x, \tau \lambda x, \kappa_{2} \lambda x\right)\right|_{x=\tilde{x}} \\
={ }^{I} \mathcal{O}_{\alpha}\left(\kappa_{1} \tilde{x}, \tau \tilde{x}, \kappa_{2} \tilde{x}\right)-\left.\tilde{x}_{\alpha} \int_{0}^{1} \mathrm{~d} \lambda \lambda^{2+I} \partial^{\mu}{ }^{I} \mathcal{O}_{\mu}\left(\kappa_{1} \lambda x, \tau \lambda x, \kappa_{2} \lambda x\right)\right|_{x=\tilde{x}}
\end{gathered}
$$

and the twist -4 vector operator which is contained in the trace terms of the twist- 3 vector operator

$$
{ }^{I} \mathcal{O}_{\alpha}^{\mathrm{tw} 4(\mathrm{ii})}\left(\kappa_{1} \tilde{x}, \tau \tilde{x}, \kappa_{2} \tilde{x}\right)=\left.\tilde{x}_{\alpha} \int_{0}^{1} \mathrm{~d} \lambda \lambda^{2+I} \partial^{\mu}{ }^{I} \mathcal{O}_{\mu}\left(\kappa_{1} \lambda x, \tau \lambda x, \kappa_{2} \lambda x\right)\right|_{x=\tilde{x}} .
$$

The determination of the antisymmetric twist-4 operator having symmetry type (iii) obtains from Young tableaux

\begin{tabular}{|l|l|l|l|}
\hline$\rho$ & $\mu_{1}$ & $\cdots$ & $\mu_{N}$ \\
\hline$\beta$ & & \\
\cline { 1 - 1 }$\alpha$ & &
\end{tabular}

and 
respectively. Again, making use of property (4.18), the result is

$$
\begin{aligned}
{ }^{I} \mathcal{O}_{[\alpha \beta]}^{\mathrm{tw} 4(\mathrm{iii})}\left(\kappa_{1} \tilde{x}, \tau \tilde{x}, \kappa_{2} \tilde{x}\right)= & \left.\int_{0}^{1} \mathrm{~d} \lambda \lambda^{2+I} \delta_{[\alpha}^{\mu}\left((x \partial) \delta_{\beta]}^{\nu}-2 x^{\nu} \partial_{\beta]}\right){ }^{I} \mathcal{O}_{[\mu \nu]}\left(\kappa_{1} \lambda x, \tau \lambda x, \kappa_{2} \lambda x\right)\right|_{x=\tilde{x}} \\
& -{ }^{I} \mathcal{O}_{[\alpha \beta]}^{\mathrm{tw} 5(\mathrm{iii})}\left(\kappa_{1} \tilde{x}, \tau \tilde{x}, \kappa_{2} \tilde{x}\right),
\end{aligned}
$$

twist- 5 part is determined by the trace namely with

$$
\begin{aligned}
& { }^{I} \mathcal{O}_{[\alpha \beta]}^{\mathrm{tw} 5(\mathrm{iii})}\left(\kappa_{1} \tilde{x}, \tau \tilde{x}, \kappa \tilde{x}\right)=-\int_{0}^{1} \mathrm{~d} \lambda \lambda^{I}\left(1-\lambda^{2}\right)\left\{x_{[\alpha}\left(\delta_{\beta]}^{[\mu}(x \partial)-x^{[\mu} \partial_{\beta]}\right) \partial^{\nu]}\right. \\
& \left.-x_{[\alpha} \delta_{\beta]}^{[\mu} x^{\nu]} \square\right\}\left.^{I} \mathcal{O}_{[\mu \nu]}\left(\kappa_{1} \lambda x, \tau \lambda x, \kappa_{2} \lambda x\right)\right|_{x=\tilde{x}} .
\end{aligned}
$$

The antisymmetric twist -5 tensor operator is given by:

$$
\begin{gathered}
{ }^{I} \mathcal{O}_{[\alpha \beta]}^{\mathrm{tw} 5}\left(\kappa_{1} \tilde{x}, \tau \tilde{x}, \kappa \tilde{x}\right)={ }^{I} \mathcal{O}_{[\alpha \beta]}^{\mathrm{tw} 5(\mathrm{ii})}\left(\kappa_{1} \tilde{x}, \tau \tilde{x}, \kappa \tilde{x}\right)+{ }^{I} \mathcal{O}_{[\alpha \beta]}^{\mathrm{tw} 5(\mathrm{iii})}\left(\kappa_{1} \tilde{x}, \tau \tilde{x}, \kappa \tilde{x}\right) \\
=-\left.\frac{1}{4} x_{[\alpha}\left(\delta_{\beta]}^{\mu}(x \partial)-x^{\mu} \partial_{\beta]}\right) \square \int_{0}^{1} \mathrm{~d} \lambda \lambda^{I}(1-\lambda)^{2}{ }^{I} \mathcal{O}_{\mu}\left(\kappa_{1} \lambda x, \tau \lambda x, \kappa_{2} \lambda x\right)\right|_{x=\tilde{x}} \\
-\int_{0}^{1} \mathrm{~d} \lambda \lambda^{I}\left(1-\lambda^{2}\right)\left\{x_{[\alpha}\left(\delta_{\beta]}^{[\mu}(x \partial)-x^{[\mu} \partial_{\beta]}\right) \partial^{\nu]}\right. \\
\left.-x_{[\alpha} \delta_{\beta]}^{[\mu} x^{\nu]} \square\right\}\left.^{I} \mathcal{O}_{[\mu \nu]}\left(\kappa_{1} \lambda x, \tau \lambda x, \kappa_{2} \lambda x\right)\right|_{x=\tilde{x}} .
\end{gathered}
$$

Finally, we obtain the complete decomposition of the antisymmetric tensor operator:

$$
\begin{aligned}
{ }^{I} \mathcal{O}_{[\alpha \beta]}\left(\kappa_{1} \tilde{x}, \tau \tilde{x}, \kappa_{2} \tilde{x}\right) & ={ }^{I} \mathcal{O}_{[\alpha \beta]}^{\mathrm{tw} 3(\mathrm{ii})}\left(\kappa_{1} \tilde{x}, \tau \tilde{x}, \kappa_{2} \tilde{x}\right)+{ }^{I} \mathcal{O}_{[\alpha \beta]}^{\mathrm{tw} 4(\mathrm{iii})}\left(\kappa_{1} \tilde{x}, \tau \tilde{x}, \kappa_{2} \tilde{x}\right) \\
& +{ }^{I} \mathcal{O}_{[\alpha \beta]}^{\mathrm{tw} 4(\mathrm{ii})}\left(\kappa_{1} \tilde{x}, \tau \tilde{x}, \kappa_{2} \tilde{x}\right)+{ }^{I} \mathcal{O}_{[\alpha \beta]}^{\mathrm{tw} 5}\left(\kappa_{1} \tilde{x}, \tau \tilde{x}, \kappa_{2} \tilde{x}\right) .
\end{aligned}
$$

The determination of the symmetric twist-4 operator having symmetry type (iv) obtains from Young tableaux \begin{tabular}{|l|l|l|l|l|}
\hline$\rho$ & $\mu_{1}$ & $\mu_{2}$ & $\cdots$ & $\mu_{N}$ \\
\hline$\beta$ & $\alpha$ & &
\end{tabular} respectively. Making use of property (4.18) the result is and \begin{tabular}{|l|l|l|l|l|}
\hline$\rho$ & $\sigma$ & $\mu_{1}$ & $\cdots$ & $\mu_{N}$ \\
\hline$\beta$ & $\alpha$ & &
\end{tabular}

$$
\begin{aligned}
& { }^{I} \mathcal{O}_{(\alpha \beta)}^{\mathrm{tw} 4(\mathrm{iv})}\left(\kappa_{1} \tilde{x}, \tau \tilde{x}, \kappa_{2} \tilde{x}\right)= \\
& \left.\quad \int_{0}^{1} \mathrm{~d} \lambda \lambda^{I}(1-\lambda)\left(\delta_{\alpha}^{\mu} \delta_{\beta}^{\nu} x^{\rho}(x \partial) \partial_{\rho}-2 x^{\mu}(x \partial) \delta_{(\beta}^{\nu} \partial_{\alpha)}\right){ }^{I} \mathcal{O}_{(\mu \nu)}\left(\kappa_{1} \lambda x, \tau \lambda x, \kappa_{2} \lambda x\right)\right|_{x=\tilde{x}} \\
& \quad-{ }^{I} \mathcal{O}_{(\alpha \beta)}^{>(\mathrm{iv})}\left(\kappa_{1} \tilde{x}, \tau \tilde{x}, \kappa_{2} \tilde{x}\right),
\end{aligned}
$$

where

$$
\begin{aligned}
& { }^{I} \mathcal{O}_{(\alpha \beta)}^{>(\mathrm{iv})}\left(\kappa_{1} \tilde{x}, \tau \tilde{x}, \kappa_{2} \tilde{x}\right)= \\
& \int_{0}^{1} \mathrm{~d} \lambda\left\{\left(2 \lambda^{2+I} x_{(\alpha} \delta_{\beta)}^{\nu} \partial^{\mu}-\lambda^{1+I}(1-\lambda) x_{\alpha} x_{\beta} \partial^{\mu} \partial^{\nu}\right){ }^{I} \mathcal{O}_{(\mu \nu)}\left(\kappa_{1} \lambda x, \tau \lambda x, \kappa_{2} \lambda x\right)\right. \\
& -\frac{1}{2}\left(\lambda^{I} g_{\alpha \beta} \partial^{\mu}+\lambda^{I}\left(1-\lambda^{2}\right) x_{(\alpha} \delta_{\beta)}^{\mu} \square-\frac{1}{2} \lambda^{I}(1-\lambda)^{2} x_{\alpha} x_{\beta} \square \partial^{\mu}\right){ }^{I} \mathcal{O}_{\mu}\left(\kappa_{1} \lambda x, \tau \lambda x, \kappa_{2} \lambda x\right) \\
& \left.+\left(\frac{1}{2} \lambda^{I} g_{\alpha \beta}(x \partial)-\lambda^{2+I} x_{(\alpha} \partial_{\beta)}-\frac{1}{2} \lambda^{2+I}(\ln \lambda) x_{\alpha} x_{\beta} \square\right)^{I} \mathcal{O}^{\rho}{ }_{\rho}\left(\kappa_{1} \lambda x, \tau \lambda x, \kappa_{2} \lambda x\right)\right\}\left.\right|_{x=\tilde{x}}
\end{aligned}
$$


Now, we use the property (4.18) and sum up the symmetric higher twist operators, which appear in the trace terms of the symmetric twist operators with Young symmetry (ii) and (iv), to a complete twist- 4 , twist -5 and twist -6 operator. The complete twist-4 operators are constructed by means of the Young tableau (i). The 'scalar part' of the twist-4 tensor operator is given by

$$
\begin{aligned}
{ }^{I} \mathcal{O}_{(\alpha \beta)}^{\mathrm{tw} 4, \mathrm{~s}}\left(\kappa_{1} \tilde{x}, \tau \tilde{x}, \kappa_{2} \tilde{x}\right)=\frac{1}{2} g_{\alpha \beta}\{ & (x \partial) \int_{0}^{1} \mathrm{~d} \lambda \lambda^{I}{ }^{I} \mathcal{O}_{\rho}^{\rho}\left(\kappa_{1} \lambda x, \tau \lambda x, \kappa_{2} \lambda x\right) \\
& \left.-\partial^{\mu} \int_{0}^{1} \mathrm{~d} \lambda \lambda^{2+I}{ }^{I} \mathcal{O}_{\mu}\left(\kappa_{1} \lambda x, \tau \lambda x, \kappa_{2} \lambda x\right)\right\}\left.\right|_{x=\tilde{x}}
\end{aligned}
$$

and the twist-4 'vector part' reads

$$
\begin{gathered}
{ }^{I} \mathcal{O}_{(\alpha \beta)}^{\mathrm{tw} 4, \mathrm{v}}\left(\kappa_{1} \tilde{x}, \tau \tilde{x}, \kappa_{2} \tilde{x}\right)=-\tilde{x}_{(\alpha}\left\{\int_{0}^{1} \mathrm{~d} \lambda \lambda^{I}\left\{\partial_{\beta)}+\frac{1}{2} x_{\beta)}(\ln \lambda) \square\right\}^{I} \mathcal{O}_{\rho}^{\rho}\left(\kappa_{1} \lambda x, \tau \lambda x, \kappa_{2} \lambda x\right)\right. \\
\left.-\int_{0}^{1} \mathrm{~d} \lambda \lambda^{I}\left\{\left(1-\lambda^{2}\right) \partial_{\beta)}+\frac{1}{4} x_{\beta)}\left(1-\lambda^{2}+2 \ln \lambda\right) \square\right\} \partial^{\mu} \mathcal{O}_{\mu}\left(\kappa_{1} \lambda x, \tau \lambda x, \kappa_{2} \lambda x\right)\right\}\left.\right|_{x=\tilde{x}} .
\end{gathered}
$$

Furthermore, the complete twist -5 vector operator is again constructed by means of the Young tableau (ii). The 'vector part' of the twist- 5 tensor operator is given by

$$
\begin{aligned}
& { }^{I} \mathcal{O}_{(\alpha \beta)}^{\mathrm{tw} 5, \mathrm{v}}\left(\kappa_{1} \tilde{x}, \tau \tilde{x}, \kappa_{2} \tilde{x}\right)=-\tilde{x}_{(\alpha} \times \\
& \left\{\frac{1}{4} \int_{0}^{1} \mathrm{~d} \lambda \lambda^{I}(1-\lambda)^{2}\left(\delta_{\beta)}^{\nu}(x \partial)-x^{\nu} \partial_{\beta)}-x_{\beta)} \partial^{\nu}\right) \square^{I} \mathcal{O}_{\nu}\left(\kappa_{1} \lambda x, \tau \lambda x, \kappa_{2} \lambda x\right)\right. \\
& -\int_{0}^{1} \mathrm{~d} \lambda \lambda^{I}\left\{\left(1-\lambda^{2}\right)\left(\delta_{\beta)}^{\nu}(x \partial)-x^{\nu} \partial_{\beta)}-x_{\beta)} \partial^{\nu}\right) \partial^{\mu}-\left(\frac{1}{2}\left(1-\lambda^{2}\right)+\ln \lambda\right) x_{\beta)} \square \partial^{\mu} x^{\nu}\right\} \\
& \left.\quad \times{ }^{I} \mathcal{O}_{(\mu \nu)}\left(\kappa_{1} \lambda x, \tau \lambda x, \kappa_{2} \lambda x\right)\right\}\left.\right|_{x=\tilde{x}} .
\end{aligned}
$$

The full twist- 6 scalar operator has Young symmetry (i). The 'scalar part' of the twist -6 tensor operator is given by

$$
\begin{aligned}
& { }^{I} \mathcal{O}_{(\alpha \beta)}^{\mathrm{tw} 6, \mathrm{~s}}\left(\kappa_{1} \tilde{x}, \tau \tilde{x}, \kappa_{2} \tilde{x}\right)=\tilde{x}_{\alpha} \tilde{x}_{\beta}\left\{\frac{1}{2} \square \int_{0}^{1} \mathrm{~d} \lambda \lambda^{I}\left(1-\lambda^{2}\right) \ln (\lambda)^{I} \mathcal{O}_{\rho}^{\rho}\left(\kappa_{1} \lambda x, \tau \lambda x, \kappa_{2} \lambda x\right)\right. \\
& -\frac{1}{4} \square \partial^{\mu} \int_{0}^{1} \mathrm{~d} \lambda \lambda^{I}(1-\lambda)^{2}{ }^{I} \mathcal{O}_{\mu}\left(\kappa_{1} \lambda x, \tau \lambda x, \kappa_{2} \lambda x\right) \\
& \left.+\partial^{\mu} \partial^{\nu} \int_{0}^{1} \mathrm{~d} \lambda \lambda^{I}(1-\lambda)^{I} \mathcal{O}_{(\mu \nu)}\left(\kappa_{1} \lambda x, \tau \lambda x, \kappa_{2} \lambda x\right)\right\}\left.\right|_{x=\tilde{x}} .
\end{aligned}
$$

Thus, together with the twist-3 part, eq. (4.27), and the twist-4 part, eq. (4.35), 
we finally obtain the complete decomposition of the symmetric tensor operator:

$$
\begin{aligned}
& { }^{I} \mathcal{O}_{(\alpha \beta)}\left(\kappa_{1} \tilde{x}, \tau \tilde{x}, \kappa_{2} \tilde{x}\right)={ }^{I} \mathcal{O}_{(\alpha \beta)}^{\mathrm{tw} 3(\mathrm{ii})}\left(\kappa_{1} \tilde{x}, \tau \tilde{x}, \kappa_{2} \tilde{x}\right)+{ }^{I} \mathcal{O}_{(\alpha \beta)}^{\mathrm{tw} 4(\mathrm{iv})}\left(\kappa_{1} \tilde{x}, \tau \tilde{x}, \kappa_{2} \tilde{x}\right) \\
& \quad+{ }^{I} \mathcal{O}_{(\alpha \beta)}^{\mathrm{tw} 4, \mathrm{~s}}\left(\kappa_{1} \tilde{x}, \tau \tilde{x}, \kappa_{2} \tilde{x}\right)+{ }^{I} \mathcal{O}_{(\alpha \beta)}^{\mathrm{tw} 4, \mathrm{v}}\left(\kappa_{1} \tilde{x}, \tau \tilde{x}, \kappa_{2} \tilde{x}\right)+{ }^{I} \mathcal{O}_{(\alpha \beta)}^{\mathrm{tw} 5, \mathrm{v}}\left(\kappa_{1} \tilde{x}, \tau \tilde{x}, \kappa_{2} \tilde{x}\right) \\
& \quad+{ }^{I} \mathcal{O}_{(\alpha \beta)}^{\mathrm{tw} 6, \mathrm{~s}}\left(\kappa_{1} \tilde{x}, \tau \tilde{x}, \kappa_{2} \tilde{x}\right) .
\end{aligned}
$$

\section{Conclusion}

Let us shortly summarize our results. Making use of the tensorial harmonic polynomials up to rank 2 we determined for the first time and in a systematic way the various contributions of definite twist for a generic bilocal 2 nd rank tensor operator $G_{\alpha \beta}\left(\kappa_{1} x, \kappa_{2} x\right)$ as well as its symmetric and antisymmetric parts $G_{(\alpha \beta)}\left(\kappa_{1} x, \kappa_{2} x\right)$ and $G_{[\alpha \beta]}\left(\kappa_{1} x, \kappa_{2} x\right)$, respectively. In addition, we determined the related vector and scalar operators which occur by truncating with $x^{\beta}$ and $x^{\alpha} x^{\beta}$ or $g^{\alpha \beta}$, respectively. All these operators are harmonic tensor functions. By projection onto the light-cone we obtained the decomposition of a generic bilocal light-ray tensor operator of 2nd rank - and its reductions to (anti)symmetric tensors as well as the related vector and scalar operators - into operators of definite twist. In order to make the main results quite obvious we summarize them in Table 1 and 2. There, we indicate the different twist contributions to $G_{\alpha \beta}\left(\kappa_{1} \tilde{x}, \kappa_{2} \tilde{x}\right)=G_{(\alpha \beta)}\left(\kappa_{1} \tilde{x}, \kappa_{2} \tilde{x}\right)+G_{[\alpha \beta]}\left(\kappa_{1} \tilde{x}, \kappa_{2} \tilde{x}\right)$ by their symmetry type (i) up to (iv) and the number of the corresponding equations of Chapt. 3 ; in the second half of the tables the contributions from the trace terms are shown.

These expressions also apply to generic trilocal light-ray operators. The only difference is that the respective values of twist increase by a definite amount which is equal for any given operator and possibly, depending on the number $I$ of external $x$-factors, by a change in the integration measure, $\mathrm{d} \lambda \rightarrow \mathrm{d} \lambda \lambda^{I}$. The applicability of our procedure which has been described in Chapt. 2 for bilocal operators rests upon the fact that the general form of the Taylor expansion (around $y=0$ ) for any of the nonlocal operators is the same: It is given by an infinite sum of terms of $N$ th order being multiplied by $x^{\mu_{1}} x^{\mu_{2}} \ldots x^{\mu_{N}}$ where each term consists of a finite sum of local operators having exactly the same tensor structure. In principle, this works also for multi-local operators of higher order.

However, there are special trilocal operators which do not immediately fit into the general scheme of Chapt. 3. An example of such operators has been considered in Chapt. 4 on the same line of reasoning as for the generic case. Its twist decomposition is obtained by restricting the expressions of the generic case to the special conditions under consideration. The results are classified in Table 3 and 4, again by indicating their symmetry type and the number of the corresponding equation. In the same manner one should proceed for other kinds of special multilocal operators like that of eq. (4.8).

The present study made also obvious how difficult an exact treatment of higher twist contributions will be. There is a complicate interplay between the higher 
Table 1: Twist decomposition of general tensor operators $G_{\alpha \beta}=G_{(\alpha \beta)}+G_{[\alpha \beta]}$

\begin{tabular}{|c|c|c|c|}
\hline Eq. & YT & $G_{(\alpha \beta)}\left(\kappa_{1} \tilde{x}, \kappa_{2} \tilde{x}\right)$ & $G_{[\alpha \beta]}\left(\kappa_{1} \tilde{x}, \kappa_{2} \tilde{x}\right)$ \\
\hline \hline 3.19 & (i) & $\tau=2$ & - \\
\hline 3.50 & $(\mathrm{ii})$ & $\tau=3$ & - \\
\hline 3.47 & $(\mathrm{ii})$ & - & $\tau=3$ \\
\hline 3.65 & (iii) & - & $\tau=4$ \\
\hline 3.73 & (iv) & $\tau=4$ & - \\
\hline 3.77 & (i) & $\tau=4$ & - \\
\hline 3.78 & (i) & $\tau=4$ & - \\
\hline 3.67 & $(\mathrm{i})$ & - & $\tau=4$ \\
\hline 3.79 & (ii) & $\tau=5$ & - \\
\hline 3.68 & (ii) & - & $\tau=5$ \\
\hline 3.80 & (i) & $\tau=6$ & - \\
\hline
\end{tabular}

Table 2: Twist decomposition of general vector operators $G_{\alpha}=G_{(\alpha \bullet)}+G_{[\alpha \bullet]}$

\begin{tabular}{|c|c|c|c|}
\hline Eq. & YT & $G_{(\alpha \bullet)}\left(\kappa_{1} \tilde{x}, \kappa_{2} \tilde{x}\right)$ & $G_{[\alpha \bullet]}\left(\kappa_{1} \tilde{x}, \kappa_{2} \tilde{x}\right)$ \\
\hline \hline 3.24 & (i) & $\tau=2$ & - \\
\hline 3.52 & $(\mathrm{ii})$ & $\tau=3$ & - \\
\hline 3.53 & (ii) & - & $\tau=3$ \\
\hline 3.58 & (i) & $\tau=4$ & - \\
\hline 3.56 & (i) & - & $\tau=4$ \\
\hline
\end{tabular}

twists resulting from different symmetry types and the corresponding trace terms. The results also suffer from the equations of motion (EOM) which are frequently used in the applications because they do not contribute between physical states [21]. The twist decomposition of these EOM operators

$$
\begin{aligned}
\operatorname{EOM}_{\mathcal{O}_{\Gamma}}\left(\kappa_{1} \tilde{x}, \kappa_{2} \tilde{x}\right) & =\bar{\psi}\left(\kappa_{1} \tilde{x}\right) \Gamma U\left(\kappa_{1} \tilde{x}, \kappa_{2} \tilde{x}\right)[\mathrm{i} \not \vec{D}-m] \psi\left(\kappa_{2} \tilde{x}\right) \\
& -\bar{\psi}\left(\kappa_{1} \tilde{x}\right)[\mathrm{i} \overleftarrow{\not D}-m] \Gamma U\left(\kappa_{1} \tilde{x}, \kappa_{2} \tilde{x}\right) \psi\left(\kappa_{2} \tilde{x}\right), \quad \Gamma=\left\{1, \gamma_{\alpha}, \sigma_{\alpha \beta}\right\}
\end{aligned}
$$

is analogous to the corresponding quark operators $\mathcal{O}_{\Gamma}\left(\kappa_{1} \tilde{x}, \kappa_{2} \tilde{x}\right)$ which is given in [6]. The only difference is that for the equation of motion operators the canonical dimension and the twist raises by one unit.

The various tensor operators which we considered here are nonlocal generalizations of local operators already considered earlier in the literature for twist- 3 , cf. [24, and twist-4, cf. 23]. There, as far as possible an explicit twist decomposition has been circumvented by truncating any of the (constant) totally symmetrized tensor indices of the local operators by some light-like vector $n^{\mu}$, i.e., by reducing 
Table 3: Twist decomposition of special tensor operators ${ }^{I} \mathcal{O}_{\alpha \beta}$ with the property $x^{\beta}{ }^{I} \mathcal{O}_{\alpha \beta} \equiv 0$

\begin{tabular}{|c|c|c|c|}
\hline Eq. & YT & type & ${ }^{I} \mathcal{O}_{\alpha \beta}\left(\kappa_{1} \tilde{x}, \tau \tilde{x}, \kappa_{2} \tilde{x}\right)$ \\
\hline \hline 4.27 & (ii) & sym & $\tau=3$ \\
\hline 4.24 & (ii) & asym & $\tau=3$ \\
\hline 4.31 & (iii) & asym & $\tau=4$ \\
\hline 4.35 & (iv) & sym & $\tau=4$ \\
\hline 4.37 & (i) & sym & $\tau=4$ \\
\hline 4.38 & (i) & sym & $\tau=4$ \\
\hline 4.27 & (i) & asym & $\tau=4$ \\
\hline 4.39 & (ii) & sym & $\tau=5$ \\
\hline \hline 4.33 & (ii) & asym & $\tau=5$ \\
\hline 4.40 & (i) & sym & $\tau=6$ \\
\hline
\end{tabular}

Table 4: Twist decomposition of special vector operators ${ }^{I} \mathcal{O}_{\alpha}$ with the property $x^{\alpha I} \mathcal{O}_{\alpha} \equiv 0$

\begin{tabular}{|c|c|c|}
\hline Eq. & YT & ${ }^{I} \mathcal{O}_{\alpha}\left(\kappa_{1} \tilde{x}, \tau \tilde{x}, \kappa_{2} \tilde{x}\right)$ \\
\hline \hline 4.29 & $(\mathrm{ii})$ & $\tau=3$ \\
\hline 4.30 & $(\mathrm{i})$ & $\tau=4$ \\
\hline
\end{tabular}

to the scalar case. To the best of our knowledge the only work where the twist decomposition of (scalar) bilocal operators has been considered is that of Balitsky and Braun [13]. However, their work is based on the external field formalism and does not have an obvious group theoretical systematics.

In principle, our procedure may be extended also to tensors of arbitrary high rank. Despite being defined by an obvious algorithm, its application to the next step, the twist decomposition of a generic 3rd rank tensor operator, will be very cumbersome. First of all, it would be necessary to determine the projection operators onto all traceless 3rd rank tensorial harmonic polynomials and, secondly, also additional Young patterns had to be taken into account. Fortunately, such kind of nonlocal operators - at least in the near future - may not be of physical relevance. Therefore, any further study in that direction seems to be reasonable only from a group theoretical point of view.

However, another observation deserves mentioning. This is the appearance of the inner derivation on the cone and its relation to the conformal group. The inner derivative not only may be used in defining the property of tracelessness of the nonlocal light-ray operators. In some cases it is possible to construct the nontrivial tensor operators by applying (products of) the inner derivative on the scalar light-ray operators. Therefore, it seems to be of immediate value for a more direct determination of the complicated expressions obtained here and for a 
simplification of our procedure.

\section{Acknowledgement}

The authors are very much indebted to D. Robaschik and S. Neumeier for stimulating discussion. M.L. acknowledges financial support by Graduate College "Quantum field theory" at Center for Theoretical Sciences of Leipzig University.

\section{A Harmonic tensor polynomials}

In this Appendix we derive the operators projecting onto the traceless part of

- completely symmetric tensors of rank $n, T_{\left(\mu_{1} \ldots \mu_{n}\right)}$,

- tensors $T_{\alpha\left(\mu_{1} \ldots \mu_{n}\right)}$ of rank $n+1$ being symmetric in $n$ of its indices,

- tensors $T_{[\alpha \beta]\left(\mu_{1} \ldots \mu_{n}\right)}$ of rank $n+2$ being symmetric in $n$ and antisymmetric in two indices, and

- tensors $T_{(\alpha \beta)\left(\mu_{1} \ldots \mu_{n}\right)}$ of rank $n+2$ being symmetric in $n$ and, independently, symmetric in two indices.

In order to construct vector and tensor polynomials corresponding to $T_{\alpha\left(\mu_{1} \ldots \mu_{n}\right)}$, $T_{[\alpha \beta]\left(\mu_{1} \ldots \mu_{n}\right)}$, and $T_{(\alpha \beta)\left(\mu_{1} \ldots \mu_{n}\right)}$ we generalize the homogeneous polynomial technique which is well-known in constructing irreducible representations of the orthogonal groups and which has been used for two-point functions by Todorov et. al. [31] and in the scalar case by Nachtmann [19]. To achieve this we use the fact that, after contracting the indices of the symmetric part with some vector $x$, the resulting scalar, vector and (anti)symmetric tensor polynomials of order $n$ obey the following conditions of tracelessness:

$$
\begin{aligned}
\square \stackrel{\circ}{T}_{n}(x) & =0, \\
\square \stackrel{\circ}{T}_{\alpha n}(x) & =0, \quad \partial^{\alpha} \stackrel{\circ}{T}_{\alpha n}(x)=0, \\
\square \stackrel{\circ}{T}_{[\alpha \beta] n}(x) & =0, \quad \partial^{\alpha} \stackrel{\circ}{T}_{[\alpha \beta] n}(x)=0, \\
\square \stackrel{\circ}{T}_{(\alpha \beta) n}(x) & =0, \quad \partial^{\alpha} \stackrel{\circ}{T}_{(\alpha \beta) n}(x)=0, \quad g^{\alpha \beta} \stackrel{\circ}{T}_{(\alpha \beta) n}(x)=0 .
\end{aligned}
$$

A solution of the first three sets of equations already have been given in Part I. Below, for the readers convenience we list them for arbitrary dimensions $D=2 h$. In Chapt. 3 they will be used for $D=4$. In addition, we also solve the last set of equations; these symmetric harmonic tensor polynomials are wanted in the consideration of symmetry type (iv) of Chapter 3.4.

(i) The solutions of eq. (A.1) are the (scalar) harmonic polynomials of order $n$ corresponding to symmetric traceless tensors of rank $n$. They are given by (see e.g. [12], Chapter IX)

$$
\stackrel{\circ}{T}_{n}(x)=H_{n}^{(2 h)}\left(x^{2} \mid \square\right) T_{n}(x)
$$


with the harmonic projection operator

$$
H_{n}^{(2 h)}\left(x^{2} \mid \square\right)=\sum_{k=0}^{\left[\frac{n}{2}\right]} \frac{(-1)^{k}(n+h-k-2) !}{4^{k} k !(n+h-2) !} x^{2 k} \square^{k} .
$$

(ii) The solutions of eqs. (A.2) are the harmonic vector polynomials $T_{\alpha n}(x)$ of order $n$ corresponding to the traceless tensors $T_{\alpha\left(\mu_{1} \ldots \mu_{n}\right)}$, i.e., $D$ harmonic polynomials transforming as a vector under $S O(D)$. They are given by

$$
\begin{aligned}
\stackrel{\circ}{T}_{\alpha n}(x)= & \left\{\delta_{\alpha}^{\beta}-\frac{1}{(h+n-1)(2 h+n-3)}(h-2+x \partial)\left[x_{\alpha} \partial^{\beta}-\frac{1}{2} x^{2} \partial_{\alpha} \partial^{\beta}\right]\right\} \\
& \times H_{n}^{(2 h)}\left(x^{2} \mid \square\right) T_{\beta n}(x) .
\end{aligned}
$$

After contraction with $x^{\alpha}$ the scalar harmonic polynomial $\stackrel{\circ}{T}_{n+1}(x)$ obtains.

(iii) The solutions of eqs. (A.3) are the antisymmetric harmonic tensor polynomials $\stackrel{\circ}{T}_{[\alpha \beta] n}(x)$ of order $n$. They are given by

$$
\begin{aligned}
\stackrel{\circ}{T}_{[\alpha \beta] n}(x)= & \left\{\delta_{[\alpha}^{\mu} \delta_{\beta]}^{\nu}+\frac{2}{(h+n-1)(2 h+n-3)}(h-2+x \partial)\left(x_{[\alpha} \delta_{\beta]}^{[\mu} \partial^{\nu]}-\frac{1}{2} x^{2} \partial_{[\alpha} \delta_{\beta]}^{[\mu} \partial^{\nu]}\right)\right. \\
& \left.-\frac{2}{(h+n-1)(2 h+n-4)(2 h+n-2)} x_{[\alpha} \partial_{\beta]} x^{[\mu} \partial^{\nu]}\right\} H_{n}^{(4)}\left(x^{2} \mid \square\right) T_{[\mu \nu] n}(x) .
\end{aligned}
$$

(iv) Now we want to determine the symmetric harmonic tensor polynomials $\stackrel{\circ}{T}_{(\alpha \beta) n}(x)$. Its general structure is as follows:

$$
\begin{aligned}
\stackrel{\circ}{T}_{(\alpha \beta) n}= & \left\{\delta_{\alpha}^{\mu} \delta_{\beta}^{\nu}+a_{n} g_{\alpha \beta} x^{(\nu} \partial^{\mu)}+b_{n} x_{(\alpha} \delta_{\beta)}^{(\nu} \partial^{\mu)}+c_{n} x_{(\alpha} x^{(\nu} \partial^{\mu)} \partial_{\beta)}\right. \\
& +d_{n} x^{2} \delta_{(\alpha}^{(\nu} \partial_{\beta)} \partial^{\mu)}+e_{n} x^{2} x^{(\nu} \partial^{\mu)} \partial_{\alpha} \partial_{\beta}+f_{n} x^{2} x_{(\alpha} \partial_{\beta)} \partial^{\mu} \partial^{\nu} \\
& \left.+g_{n} x_{\alpha} x_{\beta} \partial^{\mu} \partial^{\nu}+k_{n} x^{2} g_{\alpha \beta} \partial^{\mu} \partial^{\nu}+l_{n} x^{4} \partial_{\alpha} \partial_{\beta} \partial^{\mu} \partial^{\nu}\right\} \breve{T}_{(\mu \nu) n}(x)
\end{aligned}
$$

with the partially traceless polynomials

$$
\breve{T}_{(\mu \nu) n}(x)=\left(\delta_{\mu}^{\rho} \delta_{\nu}^{\sigma}-\frac{1}{2 h} g_{\mu \nu} g^{\rho \sigma}\right) H_{n}^{(2 h)}\left(x^{2} \mid \square\right) T_{(\rho \sigma) n}(x)
$$

It holds by construction

$$
\square \breve{T}_{(\mu \nu) n}(x)=0, \quad g^{\mu \nu} \breve{T}_{(\mu \nu) n}(x)=0 .
$$

From eqs. (A.4) the following system of linear equations for the nine unknown 
coefficients results:

$$
\begin{aligned}
& 0=(n-1) c_{n}+b_{n}+2 h a_{n} \\
& 0=d_{n}+g_{n}+(n-2) f_{n}+2 h k_{n} \\
& 0=2(h+n-2) d_{n}+c_{n}+b_{n} \\
& 0=2(h+n-2) e_{n}+c_{n} \\
& 0=2(h+n-2) f_{n}+c_{n}+2 g_{n} \\
& 0=2(h+n-2) k_{n}+a_{n}+g_{n} \\
& 0=4(h+n-3) l_{n}+f_{n}+e_{n} \\
& 0=(2 h+n) b_{n}+2(n-1) d_{n}+2 a_{n}+2 \\
& 0=(2 h+n) c_{n}+2 d_{n}+2 a_{n}+4(n-2) e_{n} \\
& 0=(2 h+n) f_{n}+2 k_{n}+d_{n}+8(n-3) l_{n}+2 e_{n} \\
& 0=2(2 h+n-1) g_{n}+4 k_{n}+c_{n}+2(n-2) f_{n}+b_{n} .
\end{aligned}
$$

Its unique solution is

$$
\begin{aligned}
& a_{n}=\frac{h+n-2}{(h-1)(h+n)(2 h+n-2)}, \\
& b_{n}=-2 \frac{h(h+n-1)-n+1}{(h-1)(h+n)(h+n-1)(2 h+n-2)}, \\
& c_{n}=-2 \frac{h+n-2}{(h-1)(h+n)(h+n-1)(2 h+n-2)}, \\
& d_{n}=\frac{h(h+n-1)-n+2}{(h-1)(h+n)(h+n-1)(2 h+n-2)}, \\
& e_{n}=\frac{1}{(h-1)(h+n)(h+n-1)(2 h+n-2)}, \\
& f_{n}=-\frac{h(h+n-4)-2(n-3)}{(h-1)(h+n)(h+n-1)(2 h+n-2)(2 h+n-3)}, \\
& g_{n}=\frac{(h(h+n-1)-n+3)(h+n-2)}{(h-1)(h+n)(h+n-1)(2 h+n-2)(2 h+n-3)}, \\
& k_{n}=-\frac{h(h+n-3)-2 n+3+\frac{1}{2}(h+n)(h+n-1)}{(h-1)(h+n)(h+n-1)(2 h+n-2)(2 h+n-3)}, \\
& l_{n}=\frac{h-3}{(h-1)(h+n)(h+n-1)(2 h+n-2)(2 h+n-3)} .
\end{aligned}
$$


In the case $D=4$ these coefficients simplify; they are given as follows:

$$
\begin{aligned}
a_{n} & =\frac{n}{(n+2)^{2}}, & b_{n} & =-2 \frac{n(n+3)}{(n+1)(n+2)^{2}}, \\
c_{n} & =-2 \frac{n}{(n+1)(n+2)^{2}}, & d_{n} & =\frac{4+n}{(n+1)(n+2)^{2}}, \\
e_{n} & =\frac{1}{(n+1)(n+2)^{2}}, & f_{n} & =-2 \frac{1}{(n+1)^{2}(n+2)^{2}}, \\
g_{n} & =\frac{n(n+3)}{(n+1)^{2}(n+2)^{2}}, & k_{n} & =-\frac{1}{2} \frac{n^{2}+3 n+4}{(n+1)^{2}(n+2)^{2}}, \\
l_{n} & =-\frac{1}{4} \frac{1}{(n+1)^{2}(n+2)^{2}} . & &
\end{aligned}
$$

This finishes the determination of the harmonic tensor polynomials being necessary for the present study. In principle, the extension of the procedure to arbitrary tensors of higher order is obvious. However, the explicit computation, without any additional information about their general properties, will be quite complicated. Let us remark that, to the best of our knowledge, such quantities have not been considered in the mathematical literature.

In contrast to the harmonic scalar polynomials which carry irreducible representations of $S O(2 h)$, the harmonic vector and tensor polynomials are not irreducible; they are only traceless. In order to obtain irreducible harmonic vector and tensor polynomials one has to (anti)symmetrize according to the possible Young patterns as we have done to construct operators with well-defined twist.

Finally, we remark that another method exists for the construction of scalar harmonic polynomials which seems to be well adapted to the problem of twist decomposition of light-cone operators. This method uses the space of homogeneous polynomials of degree $n$ on the complex cone as the carrier space for symmetric traceless tensors and its harmonic extensions [14]. However, no general theoretical frame exists for the vector and tensor case.

\section{References}

[1] BCDMS Collab., A.C. Benvenuti et al., Phys. Lett. B 237 (1990) 592;

L.W.Whitlow et al. Phys. Lett B 282 (1992) 475;

CCFR-NuTeV Collab., W.G. Seligman et al., Phys. Rev. Lett., 79 (1997) 1213 ;

E140X Collab., L.H. Tao et al., Z. Phys. C70 (1996) 387;

NM Collab., M. Arneodo et al., Nucl. Phys. B 483 (1997) 3;

E143 Collab., K. Abe et al., Phys. Rev. Lett. (hep-ph 9808028).

[2] M. Virchaux and A. Milsztajn, Phys. Lett. 274B (1992) 211;

A.V. Sidorov and M.V. Tokarev, Nuovo Cim. A110 (1997) 1401;

A.L. Kataev, A.V. Kotikov, G. Parente, and A.V. Sidorov, Phys. Lett. 417B 
(1998) 374;

A.V. Kotikov, V.G. Krivokhizhin, hep-ph/9805353;

A.L. Kataev, G. Parente, and A.V. Sidorov, hep-ph/ 9809500, hepph/9905310;

G. Ricco and S. Simula, hep-ph/9809264;

U.K. Yang and A. Bodek, Phys. Rev. Lett. 82 (1999) 2467;

A.I. Alekhin and A.L. Kataev, Phys. Lett. 452B (1999) 402;

A.I. Alekhin, Phys. Rev. D59 (1999) 114016; hep-ph/9902241; hepph/9907350.

[3] D.J. Gross and S.B. Treiman, Phys. Rev. D4 (1971) 1059.

[4] K. Kogut and D.E. Soper, Phys. Rev. D1 (1970) 2901.

[5] R.L Jaffe and X. Ji, Nucl. Phys. B375 (1992) 527;

X. Ji, Nucl. Phys. B402 (1993) 217.

[6] B. Geyer, M. Lazar, and D. Robaschik, Nucl. Phys. B559 (1999) 339;

B. Geyer, M. Lazar, and D. Robaschik, Nucl. Phys. B (Proc. Suppl.) 79 (1999) 560.

[7] J. Blümlein, B. Geyer, and D. Robaschik, Nucl. Phys. B560 (1999) 283.

[8] S.A. Anikin and O.I. Zavialov, Ann. Phys. (NY) 116 (1978) 135;

O.I. Zavialov, Renormalized Feynman Diagrams, (Nauka, Moscow, 1979), in Russian; Renormalized Quantum Field Theory (Kluwer Academic Press, Dordrecht, 1990), extended English translation.

[9] M. Bordag and D. Robaschik, Nucl. Phys. B169 (1980) 445.

[10] D. Müller, D. Robaschik, B. Geyer, F.-M. Dittes, and J. Hořejši: Wave functions, evolution equations and evolution kernels from light-ray operators of QCD, Fortsch. Phys. 42 (1994) 101; reprinted as hep-ph/9812448.

[11] A.O. Barut and R. Raczka, Theory of Group Representations and Applications PWN - Polish Scientific Publishers, Warszawa, 1977.

[12] N.Ya. Vilenkin and A.U. Klimyk, Representations of Lie groups and Special Functions, Vol. 2 (Kluwer Academic Publishers, Dordrecht 1993).

[13] I.I. Balitsky and V.M. Braun, Nucl. Phys. B311 (1988/89) 541;

see also: I.I. Balitsky, Phys. Lett. 124B (1983) 230.

[14] V. Bargmann and I.T. Todorov, J. Math. Phys. 18 (1977) 1141.

[15] V.K. Dobrev, G. Mack, V.B. Petkova, S.G. Petrova, and I.T. Todorov, Harmonic Analysis of the n-Dimensional Lorentz Group and its Applications to Conformal Quantum Field Theory, Lecture Notes in Physics, No. 63 (Springer, 1977). 
[16] V.K. Dobrev, V.B. Petkova, S.G. Petrova, and I.T. Todorov, Phys. Rev. D 13 (1976) 887;

N.S. Craigie, V.K. Dobrev, and I.T. Todorov, Ann. Phys. (N.Y.) 159 (1985) 411.

[17] V.K. Dobrev, A.Ch. Ganchev, and O.I. Yordanov, Phys. Lett. 119B (1982) 372 ;

V.K. Dobrev and A.Ch. Ganchev, Conformal operators from spinor fields: Antisymmetric tensor case, Dubna preprint E2-82-881 (1982).

[18] B. Geyer and M. Lazar, Twist decomposition of nonlocal light-ray operators and harmonic tensor functions, hep-th/9911024; to appear in: Proceedings Int. Conf. "Quantum Theory and Symmetries", Goslar, July 1999.

[19] O. Nachtmann, Nucl. Phys., B63 (1973) 237.

[20] S. Gottlieb, Nucl. Phys. B139 (1978) 125.

[21] H.D. Politzer, Nucl. Phys. B172 (1980) 349.

[22] R.K. Ellis, W. Furmanski, and R. Petronzio, Nucl. Phys. B207 (1982) 1, B212 (1983) 29;

E.V. Shuryak and A.I. Vainshtein, Phys. Lett. B105 (1981) 65, Nucl. Phys. B199 (1982) 951; Nucl. Phys. B201 (1982) 141;

R.L. Jaffe, Nucl. Phys. B229 (1983) 205;

A.P. Bukhvostov, G.V. Frolov, E.A. Kuraev, and L.N. Lipatov, Nucl. Phys. B258 (1985) 601;

A.P. Bukhvostov and G.V. Frolov, Yad. Fiz. 45 (1987) 1136 [Sov. J. Nucl. Phys. 45 (1987) 704].

[23] S. Wada, Progr. Theor. Phys. 62 (1979) 475, Nucl. Phys. B202 (1983) 201;

M. Okawa, Nucl. Phys. B172 (1980) 481, B187 (1981) 71;

S.P. Luttrell, S. Wada, and B.R. Webber, Nucl. Phys. B188 (1981) 219;

S.P. Luttrell and S. Wada, Nucl. Phys. B197 (1982) 290;

R.L. Jaffe and M. Soldate, Phys. Lett. B105 (1981) 467, Phys. Rev.D26 (1982) 49;

J.W. Qiu, Phys. Rev. D42 (1990) 30.

[24] M.A. Ahmed and G.G. Ross, Phys. Lett. 56B (1975) 385, Nucl. Phys. B111 (1976) 441;

A.P. Bukhvostov, E.A. Kuraev, and L.N. Lipatov, ZhETF 87 (1984) 37;

P.G. Ratcliffe, Nucl. Phys. B264 (1986) 493.

[25] E.V. Shuryak and A.I. Vainshtein, Nucl. Phys. B 201 (1982) 141.

[26] B. Geyer, D. Müller, and D. Robaschik, Nucl. Phys. B (Proc. Suppl.) 51 C (1996) 106; 
B. Geyer, D. Müller, and D. Robaschik, The evolution of non-singlet twist3 parton distribution functions, DESY 96-239, Proc. of Third Meeting on Prospects in Nucleon-Nucleon Spin Physics at HERA, Dubna, 1996, hepph/9611452.

[27] I.I. Balitsky, V.M. Braun, and A.V.Kolesnichenko, Nucl. Phys. B312 (1989) 509 ;

I.I. Balitsky and V.M. Braun, Nucl. Phys. B361 (1991) 93.

[28] P. Ball, V.M. Braun, Y. Koike, and K. Tanaka, Nucl. Phys. B529 (1998) 509;

P. Ball and V.M. Braun, Nucl. Phys. B543 (1999) 201.

[29] R.L. Jaffe and M. Soldate, Phys. Rev.D26 (1982) 49;

A.P. Bukhvostov, G.V. Frolov, E.A. Kuraev, and L.N. Lipatov, Nucl. Phys. B258 (1985) 601.

[30] J. Bartels, C. Bontus, and H. Spiesberger, Factorization of Twist-Four Gluon Operator Contributions, hep-ph/9908411.

[31] I.T. Todorov and R.P. Zaikov, J. Math. Phys. 10 (1969) 2014;

A.I. Oksak and I.T. Todorov, Comm. Math. Phys. 14 (1969) 271. 